EMEL ARIKAN

\title{
Single Period Inventory Control and Pricing
}

An Empirical and Analytical Study of a Generalized Model 


\section{EMEL ARIKAN}

\section{Single Period Inventory Control and Pricing}

The price-setting newsvendor model is used to address the single period joint pricing and inventory control problem. The objective is to set the optimal price and replenishment quantity of a single product in order to maximize the expected profit. Products with a short selling season and relatively long replenishment lead times such as fashion goods are the most relevant application areas of the model. The focus of the work is the generalization of the model with respect to the modeling of uncertainty in demand. The author presents an analytical and empirical study which compares different demand models with a more flexible model based on price and inventory optimization. She concludes that using a general model can increase the profits significantly.

Emel Arikan holds an M. Sc. in Industrial Engineering and a doctoral degree in Social and Economic Sciences. She worked as researcher and lecturer at the Institute for Production Management at the Vienna University of Economics and Business. Her research interests include Operations Management, Inventory Control and Pricing. 
Single Period Inventory Control and Pricing

Emel Arikan - 978-3-631-75394-1

Downloaded from PubFactory at 01/11/2019 05:32:02AM

via free access 


\section{Forschungsergebnisse der Wirtschaftsuniversität Wien}

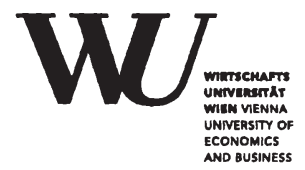

Band 43

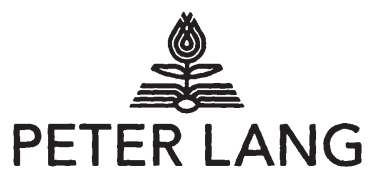

Frankfurt am Main · Berlin · Bern · Bruxelles - New York · Oxford · Wien 


\section{Single Period Inventory Control and Pricing \\ An Empirical and Analytical Study of a Generalized Model}
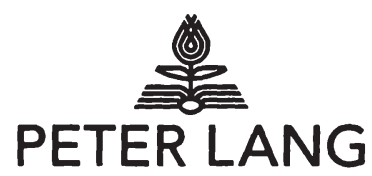

Internationaler Verlag der Wissenschaften 


\section{Bibliographic Information published by the Deutsche Nationalbibliothek}

The Deutsche Nationalbibliothek lists this publication in the Deutsche Nationalbibliografie; detailed bibliographic data is available in the internet at $h t t p: / / d n b . d-n b . d e$.

Open Access: The online version of this publication is published on www.peterlang.com and www.econstor.eu under the international Creative Commons License CC-BY 4.0. Learn more on how you can use and share this work: http://creativecommons.org/licenses/by/4.0.

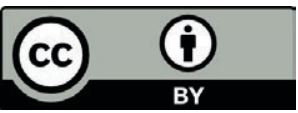

This book is available Open Access thanks to the kind support of ZBW - Leibniz-Informationszentrum Wirtschaft.

Cover design:

Atelier Platen according to a design of Werner Weißhappl.

University logo of the Vienna University of Economics and Business Administration.

Printed with kind permission of the University.

Sponsored by the Vienna University of Economics and Business Administration.

ISSN 1613-3056

ISBN 978-3-631-61222-4

ISBN 978-3-631-75394-1 (eBook)

(c) Peter Lang GmbH Internationaler Verlag der Wissenschaften

Frankfurt am Main 2011

All rights reserved.

All parts of this publication are protected by copyright. Any utilisation outside the strict limits of the copyright law, without the permission of the publisher, is forbidden and liable to prosecution. This applies in particular to reproductions, translations, microfilming, and storage and processing in electronic retrieval systems.

www.peterlang.de 


\section{Contents}

1 Introduction $\quad 11$

1.1 Joint Pricing and Inventory Control . . . . . . . . . . . 12

1.2 Research Focus . . . . . . . . . . . . . . . . 16

1.3 Structure of the Thesis . . . . . . . . . . . . . . 17

1.4 Notation and Conventions . . . . . . . . . . . . 18

2 A Review of the Newsvendor Model 21

2.1 Price-taking newsvendor model . . . . . . . . . . . . 22

2.2 Price-setting newsvendor model . . . . . . . . . . . . 24

2.2.1 Modelling demand with additive and multiplicative uncertainty . . . . . . . . . . . 2 25

2.2.2 Maximizing the expected profit . . . . . . . . . . 28

2.2 .3 Optimal price . . . . . . . . . . . . 29

3 An Empirical Study 33

3.1 Description of the data . . . . . . . . . . . . . 35

3.2 Demand estimation . . . . . . . . . . . . . . 36

3.2.1 Detrending demand data . . . . . . . . . . 36

3.2.2 Estimating the additive and the multiplicative models 37

3.3 Selection among the additive and the multiplicative models . 41

3.3.1 A formal test for model selection . . . . . . . . . . . 42

3.3.2 Selection based on homoskedasticity . . . . . . . . . 44

3.3.3 Summary of model selection . . . . . . . . . . . . . 47

3.4 Fitting a general model . . . . . . . . . . . . . . 48

3.5 Simulation of profits ................ 50

3.5.1 Comparison of the additive and the multiplicative models based on simulated profits . . . . . . . 53

3.5.2 Comparison with the general model based on simulated profits . . . . . . . . . . . . . . 54

3.5.3 Comparison of the joint and the sequential optimization based on simulated profits . . . . . . . . 56

3.5.4 Optimal policy with a limited inventory level . . . . 57

4 Analysis of the Generalized Model 63

4.1 Literature review . . . . . . . . . . . 63 
4.2 Model description . . . . . . . . . . . . . . 65

4.2.1 Failure rate and failure rate ordering . . . . . . . 67

4.2.2 Elasticity of expected sales . . . . . . . . . . 68

4.3 Non-integrated approach . . . . . . . . . . . . . . 72

4.3.1 Optimizing order quantity . . . . . . . . . . 73

4.3 .2 Optimizing price . . . . . . . . . 76

4.4 Integrated approach . . . . . . . . . . . 81

4.4.1 Optimality conditions . . . . . . . . . . . 81

4.4 .2 Structural properties . . . . . . . . . . . . . . 84

4.5 Sales elasticity for additive and multiplicative models . . . . . 86

4.6 Numerical study . . . . . . . . . . . . . . . . . . . . 89

4.6.1 Monotone variance . . . . . . . . . . . . 90

4.6.2 Non-monotone variance . . . . . . . . . . . . 98

5 Conclusion 105

$\begin{array}{ll}\text { References } & 109\end{array}$ 


\section{List of Figures}

$3.1 R^{2}$ of linear and $\log$-linear models . . . . . . . . . . . . . 40

$3.2 R^{2}$ for non-autocorrelated products . . . . . . . . . . 41

$3.3 R_{\text {comp }}^{2}$ of the linear model . . . . . . . . . . . 42

3.4 Sample variance of two example products . . . . . . . . . 47

3.5 Sample variance and coefficient of variation of an example . . 48

3.6 Sample variance and fitted variance of two example products 50

3.7 Profit improvement by using the multiplicative model instead of the additive ................. 53

3.8 Profit improvement by using the general model instead of the additive and the multiplicative models . . . . . . . 55

3.9 Profit improvement from joint optimization under the additive and the multiplicative models . . . . . . . . . . 57

3.10 Optimal price for the stochastic and deterministic models with limited inventory . . . . . . . . . . . 60

4.1 Exponential distribution function . . . . . . . . . . 65

4.2 Exponential inverse distribution function . . . . . . . . . 65

$4.3 S(p, y)$ and $\varepsilon^{p}(p, y)$ as functions of $p \ldots \ldots \ldots \ldots$

$4.4 S(p, y)$ and $\varepsilon^{y}(p, y)$ as functions of $y \ldots \ldots \ldots$

4.5 Optimal order quantity $y^{*}(p) \ldots \ldots \ldots \ldots 75$

4.6 Optimal order quantity $y^{*}(p)$ and $(\bar{p}, \bar{y}) \ldots \ldots . \ldots 85$

4.7 Mean, standard deviation, and coefficient of variation under monotone variance . . . . . . . . . . . . 91

$4.8 \varepsilon^{p}(p, y)$ and $h(p, y), M S=-2$, monotone variance $\ldots . . .92$

$4.9 \varepsilon^{p}(p, y)$ for $M S=-2$ and $V S=1$ under monotone variance . 93

$4.10 \bar{p}$ and $\bar{y}$ with respect to $V S$ under monotone variance . . . . . 94

4.11 Effect of sequential optimization with monotone variance . . 96

4.12 Effect of model misspecification with monotone variance . . . 97

4.13 Mean, standard deviation, and coefficient of variation under non-monotone variance . . . . . . . . . . . 98

$4.14 \varepsilon^{p}(p, y)$ and $h(p, y), M S=-2$, non-monotone variance . . . 99

$4.15 p^{*}(y)$ under non-monotone variance . . . . . . . . . . . 100

$4.16 \bar{p}(c)$ and $\bar{y}(c)$ when $M S=-2$ under non-monotone variance .101

$4.17 \bar{p}$ and $\bar{y}$ with respect to $V S$ under non-monotone variance . . 102

4.18 Effect of sequential optimization and model misspecification under non-monotone variance . . . . . . . . . . . . 103 
Emel Arikan - 978-3-631-75394-1

Downloaded from PubFactory at 01/11/2019 05:32:02AM

via free access 


\section{List of Tables}

1.1 Notation . . . . . . . . . . . . . . . . . . . . . 19

1.2 Probability distribution functions . . . . . . . 20

3.1 Number of products which satisfy the face validity condition . 39

3.2 Results from $\mathrm{P}_{\mathrm{E}}$-test . . . . . . . . . . . . . . . . 44

3.3 Number and percentage of heteroskedastic products $\ldots . .45$

3.4 Comparison based on tests of heteroskedasticity . . . . . . . 46

3.5 Summary of the demand distributions under the three models 51

3.6 Percentage of products where the linear model performs better than the $\log$-linear . . . . . . . . . . . . . . . 54

3.7 Average profit improvement by using the general model . . . 56

3.8 Average profit improvement of using joint optimization instead of sequential optimization, under the additive and the multiplicative models . . . . . . . . . . . . 56

3.9 Price difference between the additive stochastic and deterministic models with limited inventory . . . . . . . . . . 59

3.10 Price difference between the additive and the general models with limited inventory . . . . . . . . . . . . . . 61

3.11 Average profit improvement of using joint optimization instead of sequential optimization under the additive model with limited inventory . . . . . . . . . . . . 61 
Emel Arikan - 978-3-631-75394-1

Downloaded from PubFactory at 01/11/2019 05:32:02AM

via free access 


\section{Chapter 1 \\ Introduction}

Supply and demand management are the two crucial activities of a firm which are generally performed separately. Managing supply refers to the decisions related to the purchasing or production of goods (and capacity decisions e.g. in service industries) and is carried out by the operations/manufacturing function with the emphasis of decreasing relevant costs. On the other hand, demand management is the duty of the marketing function and includes not only observing and communicating the market demand but also influencing it by means of positioning, promotions, pricing, etc.

Traditionally, the manufacturing function is seen in a cost-center role, where its main performance indicator is unit manufacturing cost. Marketing on the other side is mainly driven by revenue targets. In this way, marketing sets prices based on a revenue optimization target and decides about the advertising policy. As reaction to that, the market creates demand which has to be satisfied by the operations at minimum cost. However, as Karmarkar and Lele (2004) points out, ignoring the interactions between these two functions may result in problems like inconsistent or even conflicting objectives and the coordination of the two can result in opportunities to improve the total system performance. Seeing these opportunities, companies put an increasing emphasis on the integration of these two organizational functions.

This research lies on the interface of these two areas, and within the large number of topics in this interface, integrated pricing and inventory decision constitutes the focus of the work. With the inventory decision we refer to the decision of how much to replenish in order to satisfy the customer demand. In a retail setting replenishment is possible through the ordering of finished goods from the suppliers while for a manufacturing firm it can as well be performed by the production of goods.

Holding inventories, on one hand, creates cost e.g. in terms of tied capital, but on the other hand make it possible to satisfy demand. It is crucial to decide on the correct amount of inventory which creates the lowest cost while keeping an acceptable service level. However, service level depends on the size of demand which in turn depends on the price of the product. The pricing decision is generally aimed at maximizing the revenue, but revenue is only possible and limited through the amount of inventory. Hence, it is 
obvious that the pricing and inventory decisions are tightly related, and they strongly influence the performance of each other. Consequently, there is an increasing effort for developing models which enable deciding on the price and ordering/production quantity of an item simultaneously.

\subsection{Joint Pricing and Inventory Control}

The degree of coordination between the marketing and manufacturing functions and their ability of working together can have considerable impact on the overall business performance. Using a survey based model Hausman et al. (2002) conclude that the harmony between the manufacturing and marketing functions is one of the major and often reinforcing factors on competitive position which is the most important predictor for profit performance.

Once the importance of marketing/manufacturing integration is conceptually established, the detailed identification of the specific conflicts as well as the areas where cooperation between those two functions is required.

Shapiro (1977) and Karmarkar and Lele (2004) provide illustrative examples about the possible problems in the interface of manufacturing and marketing activities. Shapiro (1977) describes in total eight problem fields with necessary cooperation and inherent conflicts. In particular, he points out the contradicting opinions of the two functions about the correct customer service level which is the identifier of the correct inventory level. Generally, the marketing department underestimates the costs associated with inventories while the operations department oversees the importance of customer satisfaction. Thus, if the distribution of goods to the customers is under the pure responsibility of the marketing department, while service levels are good, the inventory level is too high. Shifting the responsibility to the operations department results in lower inventory handling costs but poorer customer service. Among a number of other examples, Karmarkar and Lele (2004) discuss the effect of seasonal promotional activities on the production system. They describe a case study where the marketing department offers last minute promotions in order to meet the sales quotas and improve the sales figures which are reviewed on a quarterly basis. This sales push introduces artificial demand variation and seasonal effects which cause problems related with production planning and capacity reservation in the manufacturing function. The authors state clearly that if it were recognized that increased inventory is not an asset and if the effect on the inventory holding cost were assessed correctly in implementing the marketing strategy, then the incentive for the sales push would essentially disappear. 
Karmarkar (1996) studies the interaction and different levels of integration between marketing and operations more in-depth. He argues that "historically the interactions have been handled through mechanisms such as costs and constraints or through management process such as meetings and negotiations". However, there are several areas where these coordination mechanisms are not completely satisfactory and higher degrees of integration is necessary. He identifies the simultaneous pricing, production and inventory management as one of key issues that require full joint decision making.

The research on joint pricing and inventory control can be traced back to the seminal work of Whitin (1955). He provides an insightful discussion on the benefits of treating the two problems simultaneously for a firm facing a constant demand rate. Under the same setting, Kunreuther and Richard (1971) compares the profits when the two decisions are made sequentially and when they are made simultaneously. They show, in a numerical example, that the simultaneous decision making can increase the profit as much as $12.5 \%$.

Anticipating the benefits of joint pricing and inventory control, the attention turned to the detailed analysis of models in terms of the structure of optimal policies under different settings. The cost minimization approach of traditional inventory models and the revenue maximization approach of pricing models are now brought into a single objective of profit maximization. While for traditional inventory models, demand and price are inputs to the analytical models, for the joint optimization the effect of price on demand is considered explicitly and price becomes a decision variable.

One body of literature analyzes the problem when demand is a deterministic function of price. If demand is constant throughout the planning horizon, the models are variants of the Economic Order Quantity (EOQ) model with the explicit treatment of the price-demand relation, and for more general models the main problem is improving the lot-sizing algorithms in order to include pricing decisions. A comprehensive review of such models can be found in Yano and Gilbert (2004).

When demand is uncertain, the price-demand relation has to be described in more detail. A number of properties of demand (e.g. mean, variance, range) can be affected by price, and different formulations of these properties lead to different policies.

Under uncertain demand a number of papers consider the problem for a single period. The question is how much to order at the beginning of a single selling season and which selling price to charge. They focus mainly on the optimality conditions, the comparison of the optimal policy parameters to those under the sequential approach, and the effect of different_price-demand 
relations (see e.g. Young (1978), Petruzzi and Dada (1999), Yao et al. (2006)). They show that how the uncertainty enters into the price-demand relation has a considerable impact on the optimal policy parameters. This stream of research and this specific point of discussion is the main driver of this thesis and will be discussed in more detail in the following chapters.

For multi period models, the product is sold during a number of periods and the pricing and/or the inventory decisions are made at the beginning of each period. If there is a fixed amount of items available at the beginning of the selling season and the decision is about the pricing of the items at each period the dynamic pricing models as described by e.g. Gallego and van Ryzin (1994), and Bitran and Mondschein (1997) are relevant. A similar problem is the revenue management problem where a fixed amount of initial capacity is used to satisfy price sensitive demand which can possibly be divided into a number of classes (see e.g. Bitran and Caldentey (2003), Talluri and van Ryzin (2004)). The dynamic pricing and the revenue management problems do not require simultaneous decision making with respect to price and inventory but still can be considered as integrative approaches since they consider the level of inventory during pricing decision. A natural extension of these models is the inclusion of ordering/production decision at the beginning of the selling season. Now the initial inventory is also a decision variable yet it is fixed at the beginning of the horizon while the prices can be dynamically changed. Such models can describe the environments where the items has to be ordered before the season which is long enough to change the selling price a number of times. The typical application area of such models is the discounting/clearance periods observed extensively in the retailing industry (see e.g. Smith and Achabal (1998)).

The most general models allow a new replenishment order at each period and the price can be changed at the same time. Under such settings, the main interest is on the characterization of the optimal policy.

When there are no fixed costs associated with ordering/production the so-called base-stock list-price policy is optimal. The policy is described by an optimal base-stock level and an optimal price for each period. When the inventory level is below the optimal base-stock level, enough is ordered to bring the inventory level up to the base-stock level and the optimal list price is charged. When beginning inventory is more than the optimal base-stock level, nothing is ordered and the optimal price depends on the inventory level. Under the latter case optimal price is less than the list price, so a markdown policy is employed to deplete the inventory and reach the optimal level. Karlin and Carr (1962), Zabel (1972), Federgruen and Heching (1999) are some of the key papers in this stream of research. 
When there are fixed costs, a so called $(s, S, p)$ or $(s, S, A, p)$ policy is optimal. Under the $(s, S, p)$ policy whenever the inventory level is below $s$, enough is ordered to bring it up to $S$. Hence $s$ and $S$ refer to critical inventory levels which triggers ordering and sets the order-up-to level respectively, and the optimal price depends on the inventory level at the beginning of the period. The $(s, S, A, p)$ policy is similar to the $(s, S, p)$ policy but there might exist a set, $A$, of inventory levels where the $(s, S)$ rule does not apply. The relation between demand uncertainty and price determines which one of the two policies - $(s, S, p)$ or $(s, S, A, p)$ - is optimal. Moreover the analytical treatment of the problems also depend to a large extent on this relation, and consequently specific uncertainty models are generally analyzed separately (see Chen and Simchi-Levi (2004a), Chen et al. (2006), Song et al. (2009), Huh and Janakiraman (2008)).

The models mentioned above study the optimal actions of a single firm independent of the firms that he works or competes with. However, a popular research field and an application area of the inventory models is supply chain coordination. Issues about supply chain coordination aims at increasing the system performance of a chain by coordinating the members (e.g. suppliers and retailers) where each member tries to maximize his own benefit. A centralized supply chain can be considered as one with the highest degree of coordination, and in decentralized supply chains, when there is lack of coordination, the level of inventory kept throughout the whole chain might be different than the one in the centralized system.

Several types of contractual forms are identified as being successful in supply chain coordination when the retail price of a product is fixed and the retailer can affect sales only through the ordering/production decision (see Lariviere (1999), Cachon (2003)). However, when the retailer can also decide on his selling price, many of these contracts can not coordinate the supply chain anymore. Boyacı and Gallego (2002), Bernstein and Federgruen (2003) and Chen et al. (2001) are among many papers that consider the problem with deterministic price dependent demand and Bernstein and Federgruen (2005), Granot and Yin (2005), Granot and Yin (2007) study the uncertain demand case.

In the meanwhile a growing body of literature is directed at incorporating the customer behavior more explicitly in the models. For the multi period models, the reaction of customers to changing prices and the effects of pricing strategies on the purchasing behavior is included in a number of papers. Aviv and Pazgal (2007) considers a dynamic pricing model where the consumers develop expectations as to the availability of the product in the discount period and depending on these expectations they may postpone 
their purchases. They show that the benefits of classical dynamic pricing strategies diminishes under such strategic customer behavior. A review about the modelling of customer behavior in dynamic pricing and revenue management problems can be found in Shen and Su (2007). In addition to the pricing decisions Su and Zhang (2007) and Cachon and Swinney (2009) consider the initial inventory as a decision variable when customers are strategic. This stream of research is still quite a recent one with several promising research opportunities which includes the inventory and pricing problems.

Yano and Gilbert (2004), Chan et al. (2004) and Elmaghraby and Keskinocak (2003) provide comprehensive reviews on combined pricing and inventory models both in the single-period and the multi-period settings.

\subsection{Research Focus}

We will analyze the joint pricing-inventory problem in a single period setting under the newsvendor model. The newsvendor problem assumes that only one procurement decision is made before the beginning of the selling season and further replenishment during the period is not possible. Fashion apparel retailers who must submit orders in advance of a selling season with no opportunity for replenishment, manufacturers who have to choose the capacity before launch of a new product which will become obsolete quickly, or managers who have to decide on a special one time promotion typically face the newsvendor problem (Schweitzer and Cachon, 2000). It also has wide applicability in service industries such as airlines and hotels when the key decision is capacity. While the operational decisions about the allocation of the capacity is managed through revenue management tools, the newsvendor model can be employed for the one-time capacity decision. The shortening product life cycles and the growing share of service industries implies/supports the continuing interest in the newsvendor problem.

The price-taking newsvendor model assumes that the selling price of the product is set exogenously. The essence of the price-taking newsvendor model is matching the demand and supply by appropriately setting the inventory level in the face of uncertainty. The price-setting newsvendor model, on the other hand, assumes endogenous prices. In addition to the inventory level, he can affect the demand by appropriately setting the selling price. While price-taking newsvendor sits on the supply side of the game, price-setting newsvendor has control on both sides. While this allows a larger action space and improvement opportunities, the interaction of the system 
parameters creates a more complicated setting. The model and the results differ considerably under different modelling approaches and assumptions about the model parameters.

The focus of this work is the generalization of the price-setting newsvendor model with respect to the modelling of uncertainty. The effect of price on the demand variability has a big influence directly on the pricing strategy and this in turn affects the inventory decision. How the uncertainty is included in the demand model implies the underlying variability pattern. The existing literature is concentrated on the two specific models of uncertainty, namely the additive and the multiplicative models. However, considering only these two models in the newsvendor context has not been questioned. Moreover, because the two models have been treated separately, the optimization problem does not have a unified analysis under the two models. Our aim is to evaluate the adequacy of the additive and multiplicative demand models specifically under the newsvendor framework, and analyzing the price-setting newsvendor problem in a more generalized setting.

\subsection{Structure of the Thesis}

After defining the frequently used notation and conventions in the next Section 1.4 we give an overview of the newsvendor model in Chapter 2 mainly from a modelling approach. First, we briefly present the price-taking newsvendor model in order to introduce the basic concepts. Next, the pricesetting newsvendor model is presented in more detail focusing on the two classical demand models - additive and multiplicative.

In Chapter 3 we present an empirical study which includes demand modelling as well as price and inventory optimization. Using the sales data of a retailing company, the additive and the multiplicative demand models are estimated and their adequacy of representing the data is assessed according to some statistical methods. Seeing the need and possibility of using a more general demand model we suggest estimating a more flexible demand distribution in a simple way. Applying the newsvendor problem formulation, the optimal policies under each of the three models as well as the policy under the sequential approach are calculated. The performance of each model is evaluated by simulating the corresponding policies using the same data set. At the end, we conclude that using a general model can increase the profits significantly.

Building on the conclusion of Chapter 3 we continue with an analytical study of the price-setting newsvendor model with a general price dependent 
demand distribution in Chapter 4. First we give a review of the three papers which have similar motivations. Then we introduce our model, basic assumptions, and two important concepts - failure rate and sales elasticity - in Section 4.2. In Section 4.3 we consider the problem of optimizing order quantity and price separately, while Section 4.4 is dedicated to the joint optimization of the two policy parameters. The optimality conditions and structural properties are presented in relation to failure rate and sales elasticity.

In Section 4.6 we provide a numerical study using two examples with different demand processes which can not be covered by the additive and the multiplicative models. For each example, first we illustrate the concepts and analytical findings of the previous sections. Then, we present sensitivity analysis on the profit improvement by using the general model instead of the additive and the multiplicative models.

We conclude in Chapter 5 with a summary of the results and directions for future research.

\subsection{Notation and Conventions}

Before proceeding with the analysis we present here some of the notations and the conventions used throughout the work. In the following Table 1.1, we present the notations which are used commonly almost in all chapters, but there might appear some additional notation with the corresponding definition whenever it is necessary.

Whereever the superscript $A, M, C$, and $G$ appear the variable should be considered for the Additive, Multiplicative, Combined, and General models respectively, e.g. $X^{A}(p)$ is the random demand under additive model, and $\bar{p}^{A}$ is the joint optimal price for additive model. The superscript $d$ for deterministic refers to the setting where the optimal price is calculated using the mean demand.

We use the terms increasing/decreasing and positive/negative in the weak sense, i.e. increasing means non-decreasing, and positive means non-negative. Unless otherwise stated, for the representation of the derivatives we use the variable over which the derivative is taken as a subscript to the function, e.g.

$$
\Pi_{y}(p, y)=\frac{\partial}{\partial y} \Pi(p, y)
$$




\section{Table 1.1: Notation}

\begin{tabular}{|c|c|}
\hline$p$ & per unit selling price, $p \geq 0$ \\
\hline$c$ & per unit ordering/production cost, $c>0$ \\
\hline$v$ & $\begin{array}{l}\text { salvage value per unit of leftover, } 0 \leq v<c \text {, (salvage cost if } \\
v<0 \text { ) }\end{array}$ \\
\hline$b$ & penalty cost per unit of shortage, $b \geq 0$ \\
\hline$X(p)$ & price dependent random demand \\
\hline$F(p, x)$ & $\begin{array}{l}\text { cumulative distribution function of } X(p) \text { with } p \text { as a parame- } \\
\text { ter }\end{array}$ \\
\hline$f(p, x)$ & probability density function of $X(p)$ \\
\hline$p^{*}(y)$ & optimal price for a given $y$ \\
\hline$y^{*}(p)$ & optimal order quantity for a given price $p$ \\
\hline $\bar{p}$ & optimal price for the joint optimization i.e. $\bar{p}=p^{*}(\bar{y})$ \\
\hline $\bar{y}$ & $\begin{array}{l}\text { optimal order quantity for the joint optimization i.e. } \bar{y}= \\
y^{*}(\bar{p})\end{array}$ \\
\hline$d(p)$ & $\begin{array}{l}\text { deterministic price dependent demand function decreasing in } \\
\text { price }\end{array}$ \\
\hline$U$ & random part of demand \\
\hline$\Phi(u)$ & cumulative distribution function of $U$ \\
\hline$\phi(u)$ & probability density function of $U$ \\
\hline$h(p, x)$ & failure rate of demand \\
\hline$g(p, x)$ & generalized failure rate of demand \\
\hline$S(p, y)$ & expected sales \\
\hline$\varepsilon^{p}(p, y)$ & price elasticity of expected sales \\
\hline$\varepsilon^{y}(p, y)$ & inventory elasticity of expected sales \\
\hline
\end{tabular}

If the argument(s) of the derivative include some constants or functions, first the derivative is taken and then the argument is placed, e.g.

$$
\Pi_{y}\left(p^{*}(y), y\right)=\left.\frac{\partial}{\partial y} \Pi(p, y)\right|_{p=p^{*}(y)} .
$$

Hence, we treat $\Pi_{y}(\cdot)$ as a specific function and evaluate it at the given point. If we want to first plug $p^{*}(y)$ and then take the derivative it is explicitly written as,

$$
\frac{\partial}{\partial y} \Pi\left(p^{*}(y), y\right)
$$


Lastly, Table 1.2 shows the basic properties of the probability distributions which we use in Chapter 3 and Chapter 4.

Table 1.2: Probability distribution functions

\begin{tabular}{|c|c|c|c|c|}
\hline & Parameters & Density function & $\begin{array}{l}\text { Mean \& } \\
\text { Variance }\end{array}$ & $\begin{array}{l}\text { failure } \\
\text { rate }\end{array}$ \\
\hline Normal & $\begin{array}{l}\mu \text { location } \\
\sigma \text { scale }\end{array}$ & $\frac{1}{\sigma \sqrt{2 \pi}} \exp \left(-\frac{(x-\mu)^{2}}{2 \sigma^{2}}\right)$ & $\begin{array}{l}\mu \\
\sigma^{2}\end{array}$ & $\begin{array}{l}\text { IFR } \\
\text { IGFR }\end{array}$ \\
\hline $\begin{array}{l}\text { Log- } \\
\text { normal }\end{array}$ & $\begin{array}{l}\mu \text { location } \\
\sigma \text { scale }\end{array}$ & $\frac{1}{x \sigma \sqrt{2 \pi}} \exp \left(-\frac{(\ln (x)-\mu)^{2}}{2 \sigma^{2}}\right)$ & $\begin{array}{l}e^{\mu+\sigma^{2} / 2} \\
\left(e^{\sigma^{2}}-1\right) e^{2 \mu+\sigma^{2}}\end{array}$ & $\begin{array}{l}\text { DFR } \\
\text { IGFR }\end{array}$ \\
\hline Gamma & $\begin{array}{l}\alpha \text { shape } \\
\beta \text { scale }\end{array}$ & $x^{(\alpha-1)} e^{-(x / \beta)} \frac{1}{\beta^{\alpha} \Gamma(\alpha)}$ & $\begin{array}{l}\alpha \beta \\
\alpha \beta^{2}\end{array}$ & $\begin{array}{l}\text { IFR* } \\
\text { IGFR }\end{array}$ \\
\hline
\end{tabular}

$\left.{ }^{*}\right)$ for $\alpha>1$. IFR/DFR: Increasing/Decreasing Failure Rate, IGFR: Increasing Generalized Failure Rate 


\section{Chapter 2}

\section{A Review of the Newsvendor Model}

The newsvendor model is a single period inventory control model. The product in question is sold during one season and the ordering (or production) quantity should be set before the season starts. The demand during the selling period is not known before the season starts. Thus the ordering decision is made under uncertainty about the amount of demand. During the selling season, it is not possible to order additional units and cover the unexpected part of demand. At the end of the season unsatisfied demand is lost and the leftover inventory is obsolete.

One of the application areas of the newsvendor model is the inventory management of perishable goods such as fresh produce or newspapers. These products are naturally single period products since they have a limited useful life. Another area is the fast-changing markets with short life-cycle products such as fashion textiles (Fisher and Raman (1996)) or consumer electronics. In these industries the production and transportation lead-times are generally longer than the market lifetime of the product. In this case, it is not possible to order additional units during the selling season. Additionally, the newsvendor model is relevant to the capacity management and revenue management problems (see e.g. Van Mieghem and Rudi (2002), McGill and van Ryzin (1999)), because of the one-time irreversible nature of the decisions and the stochastic environment in these problems.

Other than its applicability, the newsvendor model provides important structural results which are not possible to derive in the multi-period setting because of the models' complexity. These results help better understand the multi-period problems since the newsvendor model is the building block of the multi-period models. From a technical point of view, the analytical treatment of the multi-period models often include dynamic programming models that rely on inductive proofs. The properties of the single period problem constitute the starting point for the analysis. Moreover, when infinite horizon problems are considered, the stationary policies often turn out to share the same characteristics with the single period solutions.

Traditionally, the newsvendor problem deals only with the inventory decision. The selling price and the corresponding demand forecast are considered as input parameters for the newsvendor's inventory decision. 
In this situation the newsvendor acts as a price-taker. However, in many situations the newsvendor has a pricing power on his product. The fashion retailers or suppliers of popular electronics products can set the price of their products and affect their demand. The class of price-setting newsvendors is the subject of this research.

\subsection{Price-taking newsvendor model}

For the price-taking newsvendor, the price of the product is an exogenous variable. It can be assumed that the newsvendor is a small player in a perfectly competitive market and has no power to influence his selling price or he is a retailer of a product for which the price is set centrally. Given that the selling price is $p$ and the purchasing cost per unit is $c$, he has to decide on the ordering quantity $y$.

Demand, $X$, is a random variable which is realized after the ordering decision. It follows a known probability distribution with a strictly increasing distribution function $F(\cdot)$, which is independent of price, and the corresponding density function $f(\cdot)$. If demand during the period turns out to be less than the ordering quantity the newsvendor ends up with some leftover inventory which can not be carried to another selling season. On the other hand, if demand is larger than the ordering quantity the unsatisfied demand is lost since there is no further replenishment option. Since there is a single selling period it is generally assumed that before the ordering decision the newsvendor does not have any initial inventory, though the positive initial inventory requires just a simple modification of the basic model.

Since demand is a random variable, the resulting profit is also a random variable which depends on the ordering quantity. If $y$ is the ordering quantity and $x$ is the demand realization, profit is

$$
\text { Profit }= \begin{cases}p x-c y & x \leq y \\ p y-c y & x>y\end{cases}
$$

The objective of the newsvendor is to find the optimum ordering quantity $y^{*}$ which maximizes the expected profit $\Pi(y)$ where

$$
\begin{aligned}
\Pi(y) & =\int_{0}^{y}[p x-c y] f(x) d x+\int_{y}^{\infty}[p y-c y] f(x) d x \\
& =(p-c) y-p \int_{0}^{y} F(x) d x .
\end{aligned}
$$


The first derivative of the expected profit function is

$$
\Pi_{y}(y)=(p-c)-p F(y) .
$$

If we set $\Pi_{y}(y)$ equal to zero and solve for $y$ we derive the optimum ordering quantity $y^{*}$ as:

$$
y^{*}=F^{-1}\left(\frac{p-c}{p}\right)
$$

where $F^{-1}$ is the inverse distribution function of demand.

$y^{*}$ is the unique maximizer of expected profit since $\Pi(y)$ is strictly concave as can be observed from the negativity of the second derivative:

$$
\Pi_{y y}(y)=-p f(y)<0 .
$$

If there is some initial inventory, because of the concavity of the objective function, the optimal policy can be easily modified as follows: if the initial inventory is smaller than $y^{*}$ it is optimal to order the difference, and if the initial inventory is larger than $y^{*}$ it is optimal not to order at all. This result is the building block for the derivation of the base-stock policy in multi-period settings.

The optimum ordering quantity satisfies a balance equation of underage and overage costs. The nominator in $(2.1), p-c$, represents the opportunity cost of losing one unit of demand because of ordering too few, so it is defined as the underage cost. Similarly, ordering one unit too much costs $c$ which is the overage cost and the denominator is the sum of the two costs. Hence the optimum ordering quantity can also be represented as follows:

$$
y^{*}=F^{-1}\left(\frac{C_{u}}{C_{u}+C_{o}}\right)
$$

where $C_{u}$ and $C_{o}$ are the underage and overage costs respectively. Even if the cost structure is different, by appropriately setting the overage and underage costs the optimum ordering quantity can be found using (2.2) given the basic properties of the model still hold. Two common extensions to the cost structure are including penalty cost and salvage value.

If each unit of leftover inventory can be sold out for a salvage value, the overage cost should be decreased by that value. If the salvage value is negative, that implies a disposal cost per unit of excess inventory. On the other hand, if there is per unit penalty cost associated with lost sales, 
underage cost can be modified such that it includes the penalty cost. In both cases the solution in (2.2) can be used to find the ordering quantity.

The ratio of the overage and underage costs in (2.2) that determines $y^{*}$ is often called the critical fractile since it gives the fractile of demand distribution which corresponds to the optimum order quantity. Under the cost structure that we assume i.e. no salvage value and no penalty cost, the critical fractile can be written as:

$$
C r=\frac{p-c}{p} .
$$

$\mathrm{Cr}$ corresponds to the cycle-service level that the newsvendor aims to reach with the order quantity where cycle-service level is the probability of matching all the demand.

The critical fractile can also be interpreted as a measure for the profitability of the product. Schweitzer and Cachon (2000) classify products as high-profit products if $C r>1 / 2$ and as low-profit products otherwise. As $\mathrm{Cr}$ increases the profit margin of the product increases and it becomes more valuable to sell one more unit. Thus, if the demand distribution remains the same, the expected profit maximizing order quantity $y^{*}$ increases as the profitability of the product increases. For example, if there are two products with the same cost structure and the same demand distribution, the order quantity of the one with the higher price should be larger.

\subsection{Price-setting newsvendor model}

In many applications, the newsvendor has the chance to price his own product at least within a specific range of prices. If this is the case, the most profitable way is to decide on the price and the ordering quantity simultaneously. The selling price $p$ becomes a decision variable and the demand is assumed to be dependent on price.

The need to consider pricing and inventory problems simultaneously was first pointed by Whitin (1955). He provides a newsvendor model with pricing assuming a stochastic price dependent demand function. He derives an optimality condition based on the relation between the expected marginal profit and the expected marginal cost. For a uniform demand distribution with a price dependent mean, he provides a closed-form expression for the optimal price, which is used to find the optimal order quantity.

The literature on the price-setting newsvendor model is mainly dominated by the demand definitions with an additive and/or multiplicative uncertainty. 
In this section we will discuss these models in detail and the more general models will be discussed in chapter 4 .

\subsubsection{Modelling demand with additive and multiplicative uncertainty}

Price dependency of demand in an environment with a stochastic demand process implies that at each price a different random variable might correspond to demand. Hence, the random demand is now represented as $X(p)$, and the distribution function as $F(p, x)$. The common practice is to represent $X(p)$ as a combination of a deterministic function and an error term. The admissible prices where $X(p)$ is defined satisfy $p_{\min } \leq p \leq p_{\max }$ where $p_{\min }$ is generally assumed to be zero or $c . p_{\max }$ is defined as the price at which expected demand is zero and it is possible that $p_{\max }=\infty . d(p)$ is a nonnegative deterministic decreasing function of price, i.e. $d(p) \geq 0$, $d^{\prime}(p)<0$, and $U$ is a random variable with distribution function $\Phi(u)$ and density function $\phi(u)$ which are independent of price.

One of the most important issues about joint pricing and inventory models is the relation of $d(p)$ and $U$. Two typical approaches are to combine the two terms in an additive or a multiplicative fashion. The additive models correspond to the models where the demand is represented as the sum of the deterministic price dependent function and the random (error) term,

$$
X^{A}(p)=d(p)+U .
$$

On the other hand, the multiplicative models refer to the product of the two terms,

$$
X^{M}(p)=d(p) U .
$$

Under the additive model, probability density function of demand has the same shape as the density of the error term but its location is changed, while under the multiplicative model, the scale is changed. The distribution and the density functions of random demand can be written in terms of the corresponding functions of the error term. For the additive model $F\left(x^{A}, p\right)=$ $\Phi\left(x^{A}-d(p)\right)$ and $f\left(x^{A}, p\right)=\phi\left(x^{A}-d(p)\right)$, and for the multiplicative model $F\left(x^{M}, p\right)=\Phi\left(x^{M} / d(p)\right)$ and $f\left(x^{M}, p\right)=\phi\left(x^{M} / d(p)\right) / d(p)$.

In the additive case, the mean value of the random term, $E[U]$, is generally assumed to be zero, and in the multiplicative case it is one. Thus, under both cases expected demand corresponds to the deterministic part, $E[X(p)]=d(p)$. It is common to assume $d(p)=a-b p$ with $a_{\text {Emel }}>0, b{ }_{-1}>0$ in the additive 
models, and $d(p)=a p^{-b}$ with $a>0, b>1$ in the multiplicative models (Petruzzi and Dada, 1999).

The main difference between additive and multiplicative models is the relation of price with the variance, $V a r$, and coefficient of variation, $C v$, of demand. Under the additive model, the demand variance is

$$
\operatorname{Var}\left(X^{A}(p)\right)=\operatorname{Var}(U)
$$

and $C v$ of demand is

$$
C v\left(X^{A}(p)\right)=\frac{\sqrt{\operatorname{Var}(U)}}{d(p)+E[U]} .
$$

Under the multiplicative model, the demand variance is

$$
\operatorname{Var}\left(X^{M}(p)\right)=d(p)^{2} \operatorname{Var}(U)
$$

and the $C v$ is

$$
C v\left(X^{M}(p)\right)=\frac{\sqrt{\operatorname{Var}(U)}}{E[U]}=C v(U) .
$$

Hence, under the additive model the demand variance is constant in price while the coefficient of variation is increasing in price. On the other hand, under the multiplicative demand model, the coefficient of variation of demand equals that of the random term, which is independent of price, but the variance of demand is decreasing in price. This difference causes different pricing strategies depending on how the uncertainty is modelled. This point is the main focus of the earlier papers on the topic.

The combination of the two models is also considered in the literature in order to have a broader range of variability patterns. The demand is modelled as:

$$
X^{C}(p)=d_{1}(p) U+d_{2}(p)
$$

If $d_{1}(p)=1$, the formulation corresponds to the additive case, and if $d_{2}(p)=0$ it corresponds to the multiplicative case. Under this model, the variance and the coefficient of variation of demand is

$$
\begin{aligned}
& \operatorname{Var}\left(X^{C}(p)\right)=d_{1}(p)^{2} \operatorname{Var}(U) \\
& C v\left(X^{C}(p)\right)=\frac{d_{1}(p) \sqrt{\operatorname{Var}(U)}}{d_{1}(p) E\left[U_{\text {Unt }} t_{A} d_{2}(p)\right.}
\end{aligned}
$$


Both $V a r$ and $C v$ depend on price, which provides a generalization to the previous two models. Young (1978) is the first paper that applies the combined demand model. However, in the succeeding works it is still preferred to consider the additive model and the multiplicative model separately with some exceptions like Chen and Simchi-Levi (2004b) in a multi-period setting.

When the uncertainty is modelled additively as $X^{A}(p)=d(p)+U$, some conditions are required to guarantee nonnegative demand even if $d(p) \geq 0$ for all $p$. For example, Yao et al. (2006) assume $U \geq 0$ even if they do not explicitly mention it and in order to avoid the infinitely large revenues, an upper bound is placed on the admissible prices, so if the revenues are increasing in price a finite optimal price is still ensured on the upper price bound.

On the other hand, Karlin and Carr (1962) does not bound the admissible prices or the error term and they mention that "Since it is tedious (though not difficult) to establish general conditions ensuring the nonnegativity of demand while at the same time retaining the assumption that $U$ is distributed independently of the exogenous variable $p, \ldots$ we shall be interested only in characterizing the properties of those solutions of the model for which the optimal policies involve positive price and ordering quantities. (We assume the existence of such solutions.)" Mills (1959), without mentioning this point, does not put any restrictions on the ranges of $U$ or $p$, so it seems like he also takes the approach of Karlin and Carr (1962).

Petruzzi and Dada (1999) use a specific demand function to study the additive model, $d(p)=a-b p(a>0, b>0)$. In order to guarantee that the positive demand is possible for some prices, they assume a finite lower bound on the error term $U$. However, while this bound ensures some positive demand, it does not guarantee the nonnegativity for all possible demand realizations in the range.

For the multiplicative model the random variable $U$ is assumed to be nonnegative without any problems mentioned for the additive model. For the combined model Young (1978) assumes that the uncertainty is not additive for sufficiently large prices in order to avoid the nonnegativity problem. However, in this case it is not possible to say the combined model also covers the pure additive model.

It should be mentioned that this problem is not a crucial issue for practical applications since the price range is anyhow limited and it is not allowed to approach to infinity. 


\subsubsection{Maximizing the expected profit}

When the price affects the demand process, the objective function of the newsvendor should be modified appropriately to include this effect.

Under the additive uncertainty model, the expected profit is written as

$$
\Pi^{A}(p, y)=\int_{-\infty}^{y-d(p)} p(d(p)+u) \phi(u) d u+\int_{y-d(p)}^{\infty} p y \phi(u) d u-c y .
$$

As can be seen from the lower bound of the first integral, we do not assume a lower bound on $U$ and write it as Karlin and Carr (1962) did. Of course, for a more precise expression the lower and, if necessary, the upper bound of the integrals can be different than (minus)infinity.

When the demand model has the multiplicative uncertainty, the expected profit is

$$
\Pi^{M}(p, y)=\int_{0}^{y / d(p)} p(d(p) u) \phi(u) d u+\int_{y / d(p)}^{\infty} p y \phi(u) d u-c y .
$$

The objective is to find the optimal policy i.e. the optimal order quantity $\bar{y}$ and the optimal price $\bar{p}$, in order to maximize the expected profit in (2.6) or (2.7) depending on the demand model. This requires a joint optimization on two decision variables. The general approach is to solve the problem in a sequential way. First the quantity (price) is fixed, the price (quantity) is optimized and the resulting price $p^{*}(y)$ (quantity $y^{*}(p)$ ) is plugged in the original expected profit function. The result is a univariate problem along the optimal price (quantity) path where the only decision variable is quantity (price). This function is then maximized with respect to quantity (price) to get the optimal quantity $\bar{y}$ for the joint maximization problem and the optimal price is then $p^{*}(\bar{y})$.

In order to ensure the uniqueness and the existence of an optimal policy a series of assumptions are necessary both on the deterministic part of demand, $d(p)$, and on the distribution of the random term, $\Phi(u)$.

The first set of assumptions are necessary for the existence of a finite optimal price for a given inventory level. If $p_{\max }$ is finite even if the optimal price turns out to be a boundary solution it is still finite. On the other hand, if $p_{\max }$ is infinite the following should hold (see Karlin and Carr (1962), Young (1978)):

$$
\lim _{p \rightarrow \infty} d(p)=\lim _{p \rightarrow \infty} p d(p)=0 .
$$


For the uniqueness of an optimal price for a given inventory level, it is generally assumed that deterministic revenue is concave in price, i.e. $2 d^{\prime}(p)+p d^{\prime \prime}(p) \leq 0$ (e.g. Young (1978), Zabel (1970)). However, this assumption implies that the models can not cover some commonly used demand functions like the isoelastic demand function $d(p)=a p^{-b}$. A slightly weaker assumption is that there exists a unique price which maximizes the deterministic profit, i.e. $(p-c) d^{\prime}(p)+d(p)$ is continuous and has a unique positive zero (Karlin and Carr (1962), Petruzzi and Dada (1999)).

The uniqueness of the optimal price-quantity couple depends on the distribution of the random term. Young (1978) shows that if $U$ has a $\mathrm{PF}_{2}$ distribution, i.e. $\phi(u)$ is log-concave, or if it has the log-normal distribution, the uniqueness property holds. Moreover the results can be extended to the combination of additive and multiplicative models. Petruzzi and Dada (1999) extends the result to the IFR distributions for the additive and the multiplicative models separately.

Yao et al. (2006) presents the most general assumptions for the multiplicative and the additive models. They employ two important concepts: the price elasticity of demand and the generalized failure rate (see chapter 4). They assume that the deterministic demand function has increasing price elasticity and the error term has strictly increasing generalized failure rate. Under these conditions both for the additive and the multiplicative models the optimal policy is unique.

\subsubsection{Optimal price}

The main focus of the papers on price-setting newsvendor problem is the structural properties of the optimal price. The inventory problem is generally considered as the result of the pricing decision and does not gain specific attention. As mentioned by Yano and Gilbert (2004), the earlier works take the deterministic demand functions as the starting point and then bring the uncertainty into question. That's why they specifically focus on the effect of including uncertainty on pricing strategy.

Mills (1959) was the first to write the demand function explicitly as an additive demand model as in (2.3) and the main consideration is to show the effect of uncertainty on the optimal price. The price which optimizes the deterministic profit function is defined as the optimal riskless price $p^{d}$ such that

$$
p^{d}=\underset{p}{\operatorname{argmax}}\{(p-c) d(p)\} .
$$

$p \quad$ Emel Arikan - 978-3-631-75394-1 
The optimal price which maximizes the expected profit with an additive demand function is

$$
\bar{p}^{A}=\underset{p}{\operatorname{argmax}}\left\{\Pi^{A}\right\} .
$$

Mills shows that the optimal price under uncertainty is always smaller than the optimal riskless price, i.e. $\bar{p}^{A}<p^{d}$. Thus, introducing uncertainty in an additive way decreases the optimal price.

On the other hand, Karlin and Carr (1962) introduce the uncertainty in a multiplicative model and the resulting pricing strategy is opposite of the one shown by Mills (1959). Under multiplicative uncertainty the optimal price is higher than the riskless price, i.e. $\bar{p}^{M}>p^{d}$, where $\bar{p}^{M}=\operatorname{argmax}_{p}\left\{\Pi^{M}\right\}$.

Young (1978) defined the demand function in a manner that combines both additive and multiplicative models, i. e. $X^{C}(p)=d_{1}(p) U+d_{2}(p)$, and verifies both results of Mills (1959) and Karlin and Carr (1962), and generalizes their results by describing the optimality conditions in terms of variance $V a r$, and coefficient of variation $C v$ :

1. If $C v$ of demand is non-increasing in price, optimal price is larger than the riskless price.

2. If $\operatorname{Var}$ of demand is non-decreasing in price, optimal price is smaller than the riskless price.

However, when we look at the conditions it can be seen that they are still restricted in terms of variability pattern. If we define variability as the combined effect of the two measures, $\operatorname{Var}$ and $C v$, the above points represent the following properties:

1. If $C v$ of demand is non-increasing, the Var can only be decreasing in price. Both measures behave in the same direction, namely they are both non-increasing.

2. If $\operatorname{Var}$ is non-decreasing, $C v$ can only be increasing in price. Hence, both measures are non-decreasing in price.

For the multiplicative model, $\operatorname{Var}$ is decreasing when $C v$ is constant, and the first pattern is even a stronger decrease in variability. Likewise, the second pattern is the variability pattern of the additive model, and even stronger. Thus, the two conditions indicate the extreme cases and not a variability pattern where $V a r$ and $C v$ behave differently or where they are not monotone in price..

Petruzzi and Dada (1999) provide an intuitive explanation to the opposite behavior of optimal price under the additive and the multiplicative models 
and its relation to variability. The main idea is that price is a measure to decrease the demand variability, but it works different under the two models. In the additive model, "it is possible to decrease the demand coefficient of variation without adversely affecting the demand variance by choosing a lower price", on the other hand for the multiplicative model, "it is possible to decrease demand variance without adversely affecting the demand coefficient of variation by choosing a higher price". As a result, it is intuitive that the optimal price should be lower in the additive model and higher in the multiplicative model than the deterministic price.

Throughout the analysis they use a transformation of the profit function by defining a safety factor $s$, and describe the optimal price as a function of $s$. For the additive case $s=y-d(p)$, and for the multiplicative case $s=y / d(p)$. In order to find a unifying condition for the additive and the multiplicative models they write the profit function as

$$
\bar{\Pi}(s, p)=(p-c) E[\text { Sales }(s, p)]-c E[\text { Leftover } s(s, p)] .
$$

If $p_{B}(s)$ is the base price that maximizes the first part of the function, i.e. $p_{B}(s)=\operatorname{argmax}_{p}(p-c) E[\operatorname{Sales}(s, p)]$, the relation between the base price $p_{B}(s)$ and the optimal price $\bar{p}$ is the same under both types of uncertainty. They show that for the additive demand model

$$
p^{d} \geq \bar{p}^{A}=p_{B}(s)
$$

and for the multiplicative demand model

$$
\bar{p}^{M} \geq p^{d}=p_{B}(s) .
$$

Thus even if the relation to the deterministic price is different under the additive and the multiplicative uncertainty, the optimal price is larger than or equal to the base price for both types of demand uncertainty.

The problem of defining the lower bound for error distribution and its consequence on the optimal price is mentioned by Van Mieghem and Dada (1999). For a specific additive demand definition $X(p)=-p+u$, they show that the optimal price can be higher or lower than the riskless price which is contradicting with the rest of the literature. They do not assume a lower bound on $u$ but they partition the state space for $u$ such that in one of the domains $u<p$ and hence demand is negative. They then assume in this domain $X(p)=0$. However, since the range of this domain depends on price the distribution of $u$ becomes dependent on price. This means, on the other hand, that the uncertainty is not additive and the result can not be 
compared to the results under additive models. This example shows the problems with defining a pure additive demand model.

While the effect of uncertainty on price is discussed commonly in the literature, there are fewer results about the effect on order quantity. The effect depends also on the cost parameters and the properties of the error distribution. Hence unifying results are not available. 


\section{Chapter 3}

\section{An Empirical Study}

The review of the price-setting newsvendor model shows that the analytical results and the structural properties of the model depend on the type of the price-variability relation. Especially the different variability patterns underlying the additive and the multiplicative models influence the pricing decision in opposite directions. While the two models present nice technical properties and ease of use, when we turn from modelling to application it becomes critical to find the most appropriate model for the underlying real demand process.

Within the inventory control literature the relevant properties of the demand process are generally assumed to be known. In case of the pricesetting newsvendor model, the parameters of the demand function and the probability distribution are considered as given and the estimation process is not treated explicitly. On the other hand, the use of additive and multiplicative models is motivated by their popularity in the marketing literature and in practice for the demand estimation process.

Especially in the marketing literature when the effect of price on the total amount of demand is considered, the most commonly used formulations are the additive and the multiplicative models. A linear regression of demand on price represents the additive model, and the linear regression of the logarithm of demand on the logarithm of price implies the multiplicative model. We will refer to the demand models which are used to represent the underlying unknown demand process as the additive and the multiplicative form while the regression models built to estimate them will be called the linear form and the log-linear form, respectively. The two forms of regression models are frequently employed for estimating the price-demand relation. In a meta analysis of econometric studies between 1960 and 1985 about price elasticity of sales Tellis (1988) reports that the linear and the log-linear forms are among the most often utilized forms. Extending the work of Tellis (1988) with studies up to 1991 , the same observation is revealed by Kalyanam (1996). However, it is important not to forget the aim of these studies and the problem for which these models are used.

The relevant models from the marketing literature are developed in order to estimate price sensitivity of demand and to come up with a pricing policy. 
The optimal prices are calculated based on expected sales which is assumed to be the same as expected demand. This implies two major assumptions; 1) the variability regarding the impact of price on demand can be captured by the functional form selection 2) the inventory level is not considered or assumed to be unlimited for the calculation of expected sales.

If the true demand process follows a multiplicative or an additive form, with a log-linear or a linear regression, the effect of price on variance can be correctly captured. Without knowing the exact structure of the underlying process the selection of the functional form for the regression model becomes critical. However, defining the selection criteria and the tests or the methods is not trivial. One way of overcoming this problem is to use more flexible methods for estimation. For example, Kalyanam (1996) suggests the use of a Bayesian mixture model where the different functional forms are considered at the same time with some appropriate probabilities and the optimal prices are calculated based on this mixture model. However, the goal is still maximizing expected revenues assuming that the expected sales is equal to the expected demand.

In such settings, modelling or forecasting mean demand is the main question, where the focus is on creating good point forecasts while the effect of variance has a secondary role. This is justifiable as long as the profits or revenues are determined just by mean demand and stockouts are not considered. The effect of variability can be then safely ignored since positive and negative deviations from mean demand can compensate in the long run. When we start considering a limited available quantity to offer to the market, positive and negative demand deviations can not compensate anymore because of stockout situations. Hence, variability of demand starts having a major impact and we need to consider expected sales instead of expected demand for optimization models.

Nevertheless, one of the motivations for using the additive and the multiplicative models in the price-setting newsvendor problem is the common usage of these models by practitioners and the claim that the two models can represent demand in an appropriate way in many cases. In this chapter we question this claim and investigate if one of the models represents the demand process of different products better than the other.

For this purpose, we present an empirical study based on sales data from a retail chain company for several consumer products. First, we check if the properties of the additive and the multiplicative models, especially in terms of price-variance relation, hold for the data at hand. In order to estimate the demand functions we use regression analysis which is the most commonly employed statistical tool for research on pricing (Brown and Dant 2008). 
Second, and more important, we analyze the effect of the different models on the resulting profit. We apply the newsvendor model for the ordering and pricing decisions by using both demand models, compare the resulting policies, and check if the better-fit model (let us define "better-fit model" as the model which represents the data better in terms of some statistical measures) leads to significantly higher profits.

It should be underlined that the main interest is the effect of these models on the expected and real profits. Fitting of the two models to the empirical data will be, of course, evaluated with statistical measures and will give the basic comparison criterion. However, if the profit implications of using the better or worse fitting model is not significant, the contradicting analytical results of the two models with respect to the pricing policy can be considered loosely in practical settings.

As a third point we suggest a more flexible form which can capture different forms of variability. The pricing and inventory policy and the profits are calculated and compared against the two classical forms.

\subsection{Description of the data}

For our analysis we use weekly sales data of an Austrian retail chain company from a number of outlets for several products on the stock-keeping unit (SKU) level. We started with 11941 products which are sold at least for 52 weeks and on average 5 units per week. The sales data do not correspond to the real demand since demand during stockouts is not recorded. However, an analysis of stockout situations on the real data shows that they occur in less than $2 \%$ of the selling periods. Therefore, the existing sales data can be considered as an indicator for demand.

At each period, the products can be sold with different prices in different outlets. However, our analysis is not on outlet level but on the aggregated sales of each product. In order to come up with a single price at a single period, a weighted average price is calculated: the price charged at each outlet is weighted by the proportion of demand in that outlet to the overall demand. For further analysis, prices are fitted to a grid of 10 equally distributed steps.

Next to sales price, the data also contain information about the number of market outlets, and a features indicator (binary information to account for the effect of advertisement, e.g. by means of newspaper supplements such as flyers and leaflets). Just for a quarter of products such advertisement efforts were utilized. As we do with price, we also take a weighted average of the 
binary feature indicator over all outlets. Therefore at the end the variable that we use for feature is no more a binary variable but continuous between 0 and 1.

Since we are interested in the variability pattern for different prices, we want to be able to calculate the variance of demand for a number of different price levels. Therefore we eliminated the products where there are less than five prices with at least five observations each. For a given price level if there are at least five observations we consider that price for calculating the demand variance, otherwise there are not enough observations to calculate the variance. On the other hand if there are less than five prices with enough observations, there are too few prices where we can calculate the variance and analyze the effect of price on variance. Because of this reason a total of 7462 products are eliminated, and finally we proceed with 4479 products for the subsequent analysis.

Note that the products of the company are in fact durable goods,so the inventory control policy can be better modelled with a multi-period problem formulation. However we use the data to test optimal pricing and inventory policies within the newsvendor framework, without comparing our results with the applied policy at the retailer.

\subsection{Demand estimation}

As we are interested in the price-demand relation we want to model demand only as a function of price. In reality, however, demand depends on a number of other factors which should be identified. By cleaning the data from these factors we can derive the price dependent part of demand. In the following subsections we describe the estimation procedure for a single product, while the same procedure is applied to each product.

\subsubsection{Detrending demand data}

The potential factors that can significantly affect demand are chosen as trend, seasonality, number of outlets and features (see Natter et al. (2007) for a discussion). As the first step, the random demand for the product is formulated as a function of these variables:

$$
D=\mathbf{z}^{T} \beta+\underset{\text { Emel Arikan - 978-3-631-75394-1 }}{X}
$$


where $\mathbf{z}$ is a column vector of normalized independent variables such that the average over all observations of each variable is zero, and $\mathbf{z}^{T}$ is its transpose,

$$
\mathbf{z}^{T}=\left(t, \sin \left(\frac{2 t \pi}{52}\right), \cos \left(\frac{2 t \pi}{52}\right), \sin \left(\frac{2 t \pi}{4.33}\right), \cos \left(\frac{2 t \pi}{4.33}\right), O, F\right) .
$$

The first term $t$ captures the time trend and the next four terms are the seasonality components, with both annual and monthly cycles, $O$ is the number of outlets and $F$ is the feature variable. The column vector $\beta$ is the coefficient vector with parameters $\beta_{1} \ldots \beta_{7}$. As it can be seen from (3.2), the regressors (the independent variables to the regression model) do not include the constant and the price, so the effects of these two are still captured in $X$. Hence, $X$ corresponds to the price dependent demand, i.e. $X(p)$.

Let $\hat{\beta}$ be the vector of sample estimates of $\beta_{i}$ for $i=1, \ldots, 7$ and let $\hat{x}_{k}$ be the residuals to estimate $x$. By using the least squares estimation method we solve

$$
D_{k}=\mathbf{z}_{k}^{T} \hat{\beta}+x_{k} .
$$

We use $k$ as an index for a single observation, where total number of observations is $N$. The optimal values for $\hat{\beta}_{i}$ are found in order to minimize $\sum_{k=1}^{N}\left(x_{k}\right)^{2}$, and they are checked for significance on a $5 \%$ level. Note that not all the regressors are used to estimate the final model. Starting from the most general model, i.e. including all the elements of $\mathbf{z}$, we eliminate the insignificant variables step by step. While there are a number of different approaches to model building, we find the general-to-simple approach good enough for our purposes. The estimates of the coefficients of the insignificant variables are set to 0 in $\hat{\beta}$.

Now $x_{k}, k=1, \ldots, N$, is the data cleaned from any effect other than price, which we need for our further analysis. Using these data we fit an additive and a multiplicative demand model again using the linear regression models and the least squares estimation method.

\subsubsection{Estimating the additive and the multiplicative models}

From this point on, we fit the prices to a grid of 10 equally distributed steps. Let $p_{\min }$ and $p_{\max }$ be the minimum and the maximum observed prices. The price grid $\rho$ has the elements $\rho_{i}=\rho_{1}, \cdots, \rho_{10}$ such that $\rho_{i}-\rho_{i-1}$ is the same for all $i$ where $\rho_{1}=p_{\min }$ and $\rho_{10}=p_{\max }$. The price for $k^{t h}$ observation, $p_{k}$, is set to the closest price in the grid. 
The additive model is formulated as,

$$
X(p)=a+b p+U^{A} .
$$

In order to estimate the coefficients $a$ and $b$ the following regression model is solved for the respective estimates $\hat{a}$ and $\hat{b}$.

$$
x_{k}=\hat{a}+\hat{b} p_{k}+\hat{u}_{k} .
$$

The multiplicative model is formulated as,

$$
X(p)=m p^{n} U^{M}
$$

and the estimates are obtained through

$$
\ln \left(x_{k}\right)=\ln (\hat{m})+\hat{n} \ln \left(p_{k}\right)+\hat{\nu}_{k} .
$$

Note that in (3.5) $\hat{\nu}_{k}$ is an estimate for $\ln \left(u^{M}\right)$.

After the estimation of the regression coefficients, they are checked to ensure that they fit with the basic theoretical assumptions. First af all, if for a given product, demand turns out to be increasing in price we do not consider that product for further analysis. While there can be some class of products (such as luxury goods) where this relation fits with the theory, given the product portfolio of the retailer that we consider, it is quite unlikely to come up with such products. Moreover the pricing strategy of such products are not covered yet by the price-setting newsvendor literature and therefore is out of our scope.

For the linear model, if $\hat{b}$ turns out to be positive demand is increasing in price. Therefore, the linear form is not considered for comparison and in the optimization problem for all those products where $\hat{b}$ is not significantly smaller than zero. There are 3223 products, or $72 \%$ of all products where the regression analysis was applied, which results in a linear demand function decreasing in price.

For the log-linear model, if $\hat{n}$ turns out to be positive, demand is increasing in price. Moreover, if $-1 \leq \hat{n} \leq 0$ demand is inelastic to price changes. As a result, the model results in a profit which is monotonically increasing in price. When the only intention is making point estimates of demand or deriving properties of demand elasticities, $\hat{n}$ does not need to be elastic. However, when the problem is optimizing prices using the estimates, elasticity is required in order to be able to get finite optimal prices. This problem with the log-linear model is also mentioned and further discussed by Montgomery 
Table 3.1: Number of products which satisfy the face validity condition

\begin{tabular}{crr}
\hline Model & Num. products & \% of prod. \\
Additive & 3223 & 71.9 \\
Multiplicative & 2343 & 52.3 \\
Both & 2319 & 51.7 \\
None & 1232 & 27.5 \\
\hline
\end{tabular}

and Bradlow (1999). Hence, if $\hat{n} \geq-1$, the log-linear form is not considered for further analysis. Quite a high number of products, namely 2136 products, fall in this case. This corresponds to $48 \%$ of the products which are considered for the regression analysis.

These criteria about the two models can be considered as the face validity conditions, since the results are compared with theoretical expectations. A total of 1232 products fail to satisfy any of these conditions and, therefore, these products are not considered for further analysis. On the other hand, 2319 products satisfy the conditions for both of the models. The number and percentage of products satisfying the conditions for the two models are given in Table 3.1.

One of the main problems with time series data is the autocorrelation of the residuals across periods. A common cause of autocorrelation is model misspecification (Verbeek, 2008, p.105). In marketing, autocorrelation from model misspecification is generally the result of omission of a relevant explanatory variable (Hanssens et al., 2003, p.215). For example, the prices of complementary and substitutable products might have a significant effect on demand. However, since we do not know about the interrelations between products we are not able to model these effects, which might cause the misspecification problem.

In order to check for autocorrelation we apply the Durbin-Watson test which is a popular test for first-order autocorrelation (Verbeek, 2008). For the model in (3.4) the test statistic is:

$$
d w=\frac{\sum_{k=2}^{N}\left(\hat{u}_{k}-\hat{u}_{k-1}\right)^{2}}{\sum_{k=1}^{N} \hat{u}_{k}^{2}} .
$$

A non-autocorrelated process results in a test statistic $d w=2$. Hence, we test for $d w$ being significantly different from 2 . We do not check for the higher-order autocorrelation since generally the amount of autocorrelation diminishes with the lag (Ledolter and Abraham, 2006), and the seasonality 

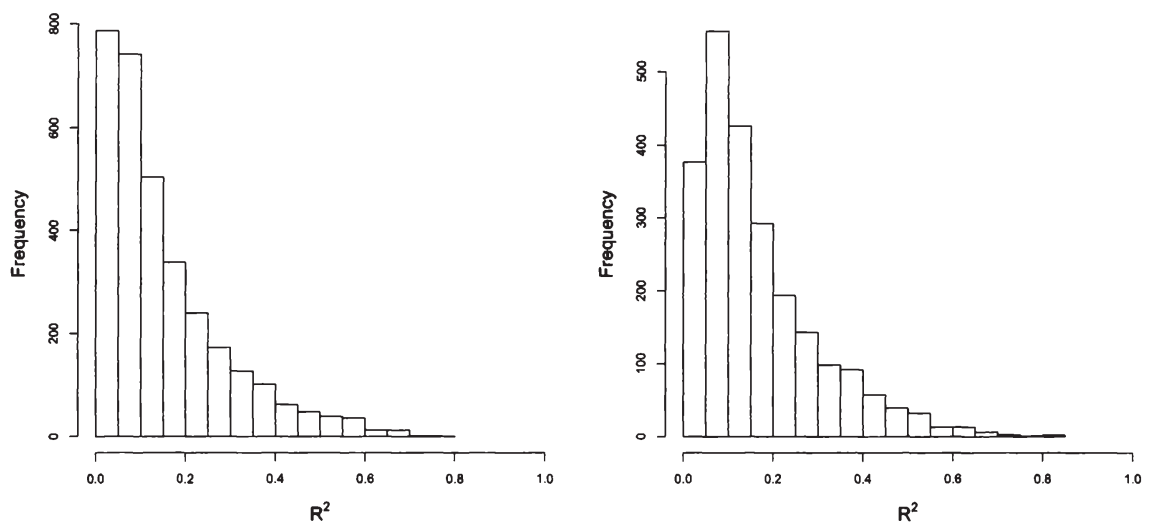

Figure 3.1: Histogram of $R^{2}$ of the linear (left) and the log-linear (right) models

is cleared in the detrending step which can help reduce the possible seasonal autocorrelation.

The results of the Durbin-Watson test for each model is as follows: out of the products satisfying the face validity for the additive model, 1092 $(33.9 \%)$ are non-autocorrelated. For the multiplicative model, $623(26.6 \%)$ products satisfying the face validity are non-autocorrelated. $550(23.7 \%)$ products satisfy the face validity of both models and at the same time are non-autocorrelated. For the rest of the chapter, we first report the results for all the products that pass the face validity check followed by the results for the non-autocorrelated products (which will be denoted as "nac.").

For all products that passed the face validity check, the histogram of the $R^{2}$ measures are given in Figure 3.1. While it seems like the $R^{2}$ values for the log-linear model are generally higher compared to the linear one, we should take into consideration that the linear model is estimated for a larger number of products compared to the log-linear model. There are 852 products with $R^{2} \geq 0.2$ for the linear model, while it is 693 for the log-linear. These numbers correspond to $27 \%$ of the products for which the linear model is estimated and $29 \%$ for the log-linear model. When we consider just the nac. products, the picture is not very different. Figure 3.2 shows the histogram for the $R^{2}$ values just for the non-autocorrelated products.

The $R^{2}$ values seem to be quite low for both models, but the reason is not the inadequate fit of the estimates to the real demand data but the fact that we are just looking at the price dependent part. Let $R_{-89 m p}^{2}$ denote the 

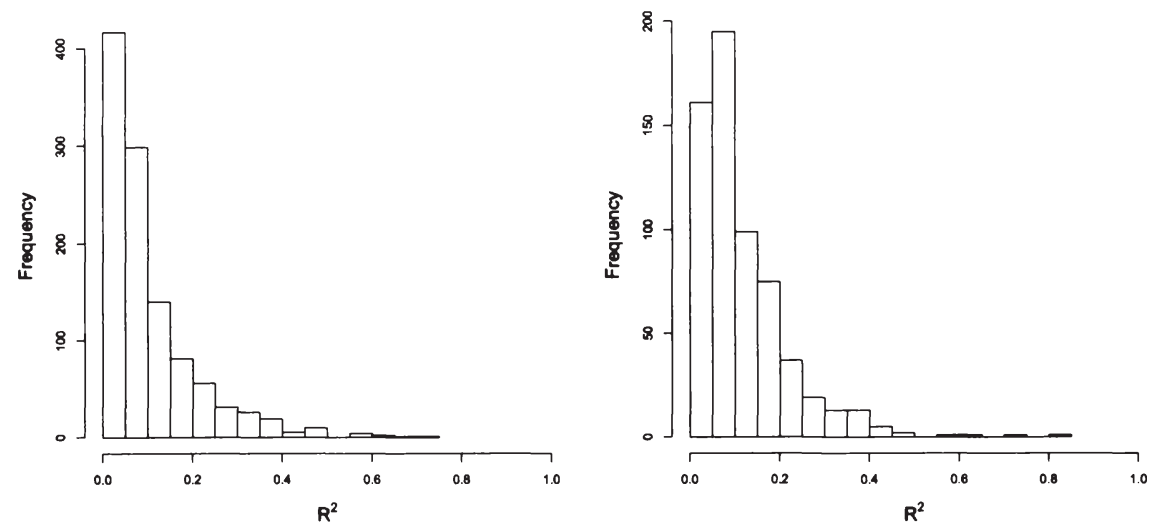

Figure 3.2: Histogram of $R^{2}$ of the linear (left) and the log-linear (right) models for nac. products

$R^{2}$ when we take the complete estimate and compare it with the original demand. If we consider the $R_{\text {comp }}^{2}$ values we see that they are indeed quite reasonable.

For the additive model we calculate the complete fit of the estimates as

$$
R_{\text {comp }}^{2}=1-\frac{\sum_{k=1}^{N} \hat{u}_{k}}{\sum_{k=1}^{N}\left(D_{k}-\bar{D}\right)^{2}} .
$$

The histogram of $R_{\text {comp }}^{2}$ is given in Figure 3.3 which shows an obvious improvement over the $R^{2}$ values in Figure 3.1 and Figure 3.2.

\subsection{Selection among the additive and the multiplicative models}

When both the linear and the log-linear forms are candidates for the demand model an important question is which one to select since the two forms will obviously suggest different pricing and inventory policies. Hence the models should be compared against each other in order to identify the most representative model. While for a small number of products it is possible to manually look at many aspects in detail, once we have a realistic assortment 

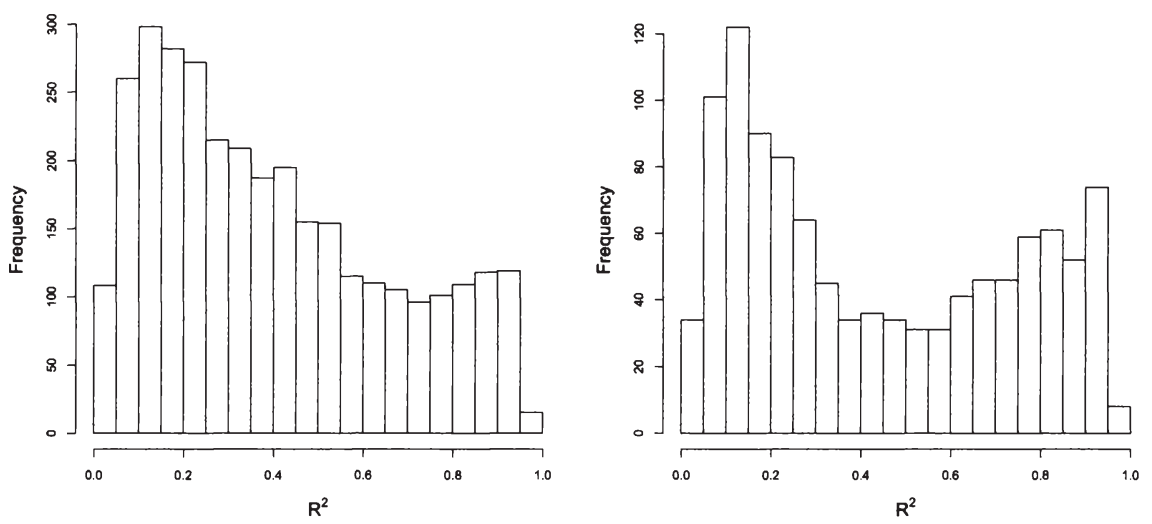

Figure 3.3: $R_{\text {comp }}^{2}$ of the linear model for all products (left) and for nac. products (right)

size with thousands of products we need to select the criteria to base our decision on.

Since the dependent variables of the two models are not the same, it is not appropriate to use $R^{2}$ directly or the likelihood function values as criteria for comparison. One possible way of comparison is transforming the estimate of the log-linear model back to the original demand level and calculating the $R^{2}$ based on this estimate. However, the transformation is not very straight-forward. One has to consider the distribution of the error term and the distribution on the estimated coefficients. Thus the result is sensitive to the distributional assumptions and there is no clear convenient way of doing a comparison on $R^{2}$.

First we employ a standard procedure for comparing linear and log-linear models using a general purpose test for model selection. Next, since a critical issue in our work is the price-variability relation, we base the model comparison on how good the demand variance is captured by each functional form.

\subsubsection{A formal test for model selection}

One of the two typical approaches for comparing linear and log-linear forms is nesting them under a more general Box-Cox transformation and comparing them against this general model (see e.g. Davidson and MacKinnon $(2004$, 
p.432)). The second approach is to create an artificial regression model using the two models such that both are nested under this artificial model. In this section we take the latter approach and use the $P_{E}$-test suggested by MacKinnon et al. (1983) for the comparison.

The $\mathrm{P}_{\mathrm{E}}$-test procedure is based on estimating an artificial compound model from the two competing models and testing the null hypothesis $H_{0}$ which states that the correct model is the linear model

$$
H_{0}: x_{k}=a+b p_{k}+u_{k}
$$

against the alternative $H_{1}$ which says that the correct model is the log-linear model

$$
H_{1}: \ln \left(x_{k}\right)=\ln (m)+n \ln \left(p_{k}\right)+\nu_{k} .
$$

Based on the two models the artificial compound model is generated as

$$
\left.x_{k}=\left(a+b p_{k}\right)+\alpha\left\{\left(\ln (\hat{m})+\hat{n} \ln \left(p_{k}\right)\right)-\ln \left(\hat{a}+\hat{b} p_{k}\right)\right)\right\}+\zeta
$$

The parameters $\hat{a}$ and $\hat{b}$ are the estimates from the linear model in equation (3.4) and $\hat{m}$ and $\hat{n}$ are the estimates from the log-linear model in equation (3.5). The idea is estimating $\alpha$ and reaching a conclusion based on this estimate with a standard t-test. If $\alpha$ is significantly different from zero, the linear model is rejected, but this does not mean that the log-linear model has to be accepted. As an opposite case, if $\alpha$ does not turn out to be significantly different from zero, then the linear model is not rejected, but again, we can not yet conclude that the log-linear model is not also true. Up to this point the test is not very conclusive, hence the same procedure is repeated after exchanging the null and the alternative hypothesis. Now $H_{0}$ corresponds to the log-linear model and $H_{1}$ to the linear model and the artificial compound model is also reformulated accordingly as

$$
\ln \left(x_{k}\right)=\left(\ln (m)+n \ln \left(p_{k}\right)\right)+\alpha\left\{\left(\hat{a}+\hat{b} p_{k}\right)-\left(\hat{m} p_{k}^{\hat{n}}\right)\right\}+\zeta
$$

At the end the test might give one of the four possible results as shown in Table 3.2. As can be seen, the problem with the $\mathbf{P}_{\mathbf{E}}$-test is that in two cases (type 3 and 4) the result is not conclusive. The test is not able to really compare the two models against each other but "the goal is to assess the 'truth' of each model's specification and categorically accept or reject each of the competing alternatives" (Balasubramanian and Jain, 1994).

Table 3.2 includes the number of products which fall into each category for all products and for the non-autocorrelated products, respectively. Un- 
Table 3.2: Results from $P_{E}$-test

\begin{tabular}{clrr}
\hline Type & Conclusion drawn & Nr. and \% of products \\
& & all & nac. \\
\hline 1 & Accept linear, reject log-linear & $281(12.1 \%)$ & $55(10.0 \%)$ \\
2 & Reject linear, accept log-linear & $282(12.2 \%)$ & $49(8.9 \%)$ \\
3 & Reject both linear and log-linear & $598(25.8 \%)$ & $69(12.5 \%)$ \\
4 & Reject neither linear nor log-linear & $1158(49.9 \%)$ & $377(68.5 \%)$ \\
\hline
\end{tabular}

fortunately many of the products are of type 3 or 4 , which are not very conclusive. Moreover, the number of products of type 1 and 2 are very close. Hence, the conclusion from this test can only be that a priori neither of the models is more preferable against the other one.

Similar results are reported by Bolton (1989) and Kalyanam (1996). Bolton (1989) compares the models based on the transformed $R^{2}$ values while he does not comment on how the transformation was done. He concludes that the average $R^{2}$ is approximately equal across models. However, the different functional forms have different systematic bias with respect to the estimates. Therefore it is crucial to test the alternative forms. On the other hand Kalyanam (1996) compares the log-linear model with a semi-log model, i.e. a model in the form of $\ln \left(x_{k}\right)=\beta_{0}+\beta_{1} p+\epsilon$, by using the P-test which is based on an artificial compound model like the $\mathrm{P}_{\mathrm{E}}$-test. He faces the same problem of the low power of the test since for 5 out of 6 comparisons the result is of type 4 , so no conclusion can be made.

\subsubsection{Selection based on homoskedasticity}

While the $\mathrm{P}_{\mathrm{E}}$-test is a formal way of comparing the linear and the log-linear models in order to find the true model, evaluating them based on a specific criterion can be another legitimate way of comparison. In this section, we test how good the models represent the demand variance and use this as a criterion for model selection.

Recall that within the price-setting newsvendor model, the main effect of using different demand models come from the different variability patterns implied by these models. Under the additive model demand variance is assumed to be independent of price, and under the multiplicative one the coefficient of variation should be price independent. In order to investigate these two properties, we analyze the variance of the residuals of the two regression models in (3.4) and (3.5). If the corresponding residuals, $\hat{u}_{k \text { E }}$ and/or 
Table 3.3: Number and percentage of heteroskedastic products

\begin{tabular}{l|rr|rc}
\hline \multirow{2}{*}{ Model } & \multicolumn{2}{|c|}{ All products } & \multicolumn{2}{c}{ Nac. products } \\
& B-P test & White test & B-P test & White test \\
\hline linear & $1566(48.6 \%)$ & $1435(44.5 \%)$ & $443(40.6 \%)$ & $384(35.2 \%)$ \\
log-linear & $546(23.3 \%)$ & $635(27.1 \%)$ & $93(14.9 \%)$ & $100(16.1 \%)$ \\
\hline
\end{tabular}

$\hat{\nu}_{k}$, are homoskedastic, i.e. the variance of the residuals is identical through the observations, the assumptions of the additive and/or the multiplicative models are fulfilled. We check for homoskedasticity of the residuals by using some standard tests, namely the Breusch-Pagan test (B-P test) and the White test. Both of these tests aim to identify the heteroskedasticity which might be caused by one of the independent variables.

The test by Breusch and Pagan (1979) is based on an auxiliary regression of the squared residuals on a function of price. If we consider the additive case, the auxiliary regression is:

$$
\sigma_{k}^{2}=\sigma^{2} \mathfrak{f}\left(\alpha_{0}+\alpha p_{k}\right)
$$

where $\sigma^{2}$ is a constant independent of the observation and $f$ is any general function. If $\alpha=0, \sigma_{k}^{2}$ is the same for all observations and the model is homoskedastic. Hence, the null and alternative hypotheses are:

$$
\begin{aligned}
& H_{0}: \alpha=0 \\
& H_{1}: \operatorname{Not} H_{0} .
\end{aligned}
$$

The test requires the specification of $\mathfrak{f}$ and for this purpose we choose the simplest variant, where $f$ is a linear function in $p$. Since the Breusch-Pagan test is quite sensitive to the assumption of normality, we use the extension introduced by Koenker (1981) and Koenker and Bassett (1982).

The test by White (1980) is a generalization of the B-P test such that $f$ is a linear function of $p$ and $p^{2}$. Hence, it can cover non-monotone variance changes in price. While this generalization offers an advantage, it results in a limited power of the test.

The results of the two tests are reported in Table 3.3. Note that the tests were applied to the products which satisfy face validity, so the number of products under the linear and the log-linear models differ (see Table 3.1). When we look at the percentages, it can be concluded that the log-linear model seems to capture the price-variance relation better than the linear 
Table 3.4: Comparison based on tests of heteroskedasticity: number and percentage of products in each type

\begin{tabular}{cllrrrrr}
\hline \multicolumn{7}{c}{ All products } \\
\hline Type & Linear & Log-linear & \multicolumn{1}{c}{ B-P test $\left(\%^{1}\right)$} & White test $\left(\%^{1}\right)$ \\
\hline 1 & Homosked. & Heterosked. & 271 & $(18.0 \%)$ & 248 & $(18.1 \%)$ \\
2 & Heterosked. & Homosked. & 964 & $(64.1 \%)$ & 740 & $(54.1 \%)$ \\
3 & Heterosked. & Heterosked. & 268 & $(17.8 \%)$ & 381 & $(27.8 \%)$ \\
4 & Homosked. & Homosked. & 817 & - & 950 & - \\
\hline \hline & & \multicolumn{7}{c}{ Nac products } & & & \\
\hline Type & Linear & Log-linear & B-P test $\left(\%{ }^{1}\right)$ & White test $\left(\%{ }^{1}\right)$ \\
\hline 1 & Homosked. & Heterosked. & 46 & $(16.5 \%)$ & 39 & $(16.1 \%)$ \\
2 & Heterosked. & Homosked. & 197 & $(70.9 \%)$ & 160 & $(66.1 \%)$ \\
3 & Heterosked. & Heterosked. & 35 & $(12.6 \%)$ & 43 & $(17.8 \%)$ \\
4 & Homosked. & Homosked. & 272 & - & 308 & - \\
\hline
\end{tabular}

${ }^{1}$ Percentage values are calculated based on the sum of products of type 1,2 , and 3 .

model. However, such an interpretation should be taken cautiously since the aim and the structure of these tests are not about comparison of functional forms.

For the products which satisfy the face validity for both models, we can have a more detailed look in Table 3.4. While the tests are not comparing the two models, we use the results to interpret them for a comparison with respect to the truth of the model as we did with the $\mathrm{P}_{\mathrm{E}}$-test in the previous section. In Table 3.4 type 1 and 2 recommend using the linear or the loglinear model, respectively, if the only criterion would be about variance. Type 3 on the other hand includes all those products where neither the linear nor the log-linear model is able to capture the full price-variance relation. Type 4 products include all those where both the linear and the log-linear model result in homoskedastic residuals. Although this last category does not sound to be very intuitive, one of the following points can be the explanation:

1. The demand and its variance does not significantly depend on price.

2. Because of the low power of the tests the null hypothesis of homoskedasticity is not rejected.

3. Due to the range of the observed prices and demands, the log-linear model might look very similar to the linear one and hence, the test is not able to differentiate between them. 

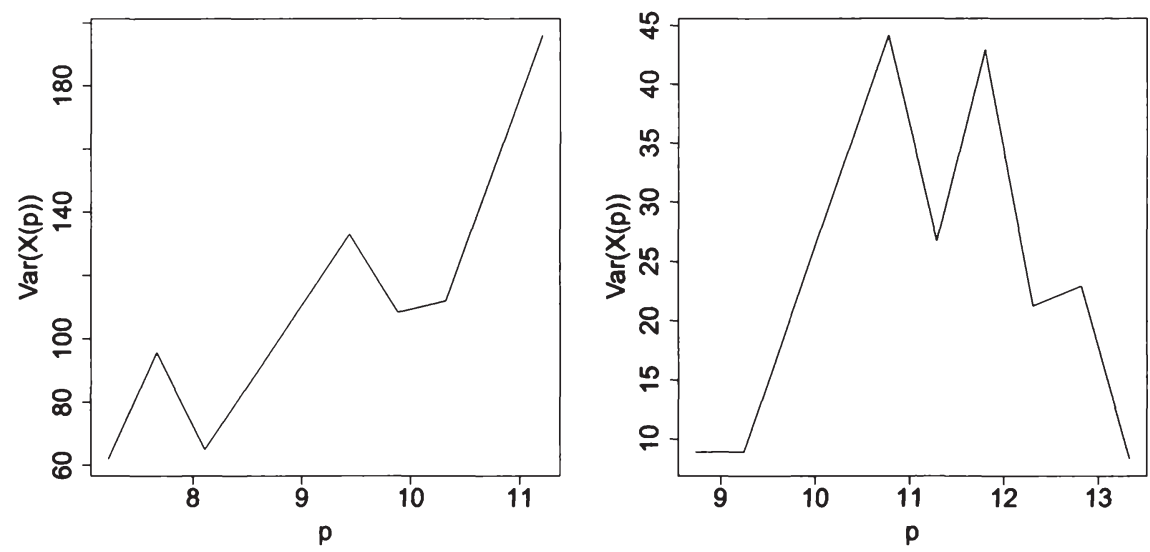

Figure 3.4: Sample variance of two example products

4. The variance in fact depends on price in both cases but can not be captured with a simple linear relation as in the B-P test or with a second order polynomial as in the White test.

Since the type 4 result is quite vague and inconclusive, we do not include it in the calculation of the percentages. When we consider the first three types we can conclude that the log-linear model is again performing better than the linear one, which is in line with many findings.

The results are slightly different for the non-autocorrelated products as the proportion of type 3 products is smaller while the type 2 is higher for these products. Nevertheless the conclusion is not different than the conclusion considering all of the products: the log-linear model performs better than the linear but for many products it is not possible to conclude for one of the models.

\subsubsection{Summary of model selection}

The comparisons based on both the $\mathrm{P}_{\mathrm{E}}$-test and homoskedasticity result in a large class of products where it is not possible to choose among the linear and the log-linear models. First of all, there are a number of products which fall in type 3 both in Table 3.2 and Table 3.3 for which neither of the models seem to be appropriate. In Figure 3.4 we plot the variance of demand for two of these products. The first product does clearly not fit with the assumptions of neither of the models since it has an increasing variance, 

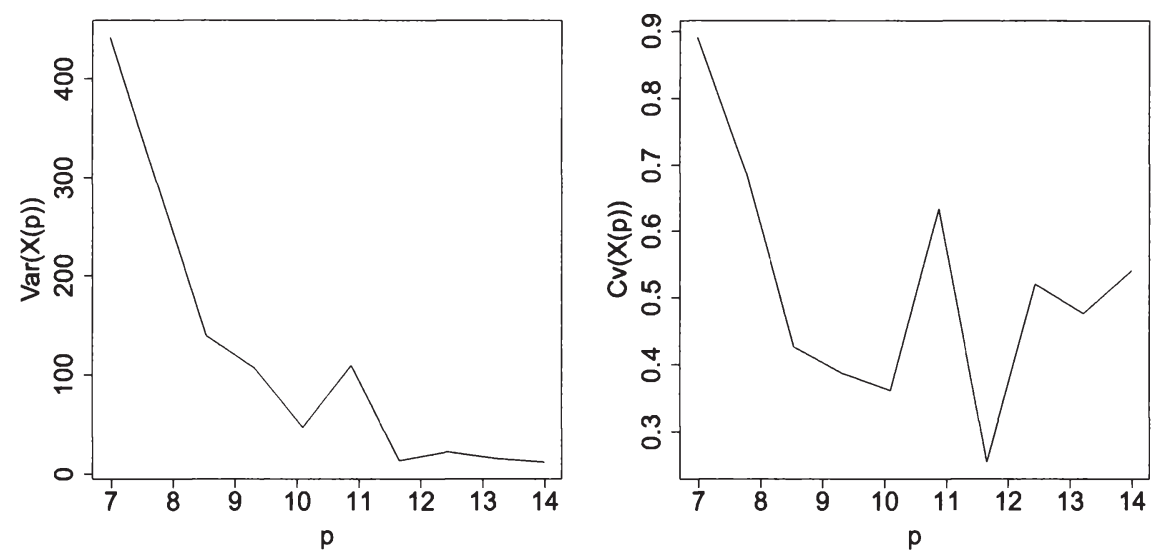

Figure 3.5: Sample variance and coefficient of variation of an example product

while the second one seems to imply a non-monotone price-variance relation. Therefore it is no surprise that they are identified as heteroskedastic under both the linear and log-linear forms.

Another problematic class of products is that one, where the log-linear model is identified as homoskedastic (i.e. type 2 in Table 3.3) because the variance is decreasing in price, while the coefficient of variation in fact does not fit with the multiplicative model. Figure 3.5 shows the Var and $\mathrm{Cv}$ of one of these products. Since $\operatorname{Var}(X(p))$ is decreasing in $p$, both the B-P test and the White test identified it as homoskedastic under the log-linear model. Hence, one can assume that the findings from the multiplicative model would apply for this product. However, this might be a misleading conclusion because $C v(X(p))$ is not really constant in $p$ as implied by the multiplicative model.

\subsection{Fitting a general model}

As the previous discussion and analysis remained unsatisfactory with respect to several aspects, we see the need for a more general and flexible demand definition. There are numerous products where the adequacy of the linear and the log-linear forms are questionable, so we try to model demand in a way that we can cover the properties of demand for products as in Figure 3.4 and 3.5. Since our aim is to find the optimal price and inventory level with the newsvendor model we do not need a point estimate but a whole distribution. 
Therefore, already at the estimation step focusing on the distribution seems to be reasonable. For now, we leave the discussion of which distribution to choose and focus on the specification of the two parameters the mean and the variance of the distribution.

We suggest estimating the variance and the mean of demand for each price separately, and using directly the distribution function with these parameters. Since we want to be able to capture a more general price-variance relation we model $\operatorname{Var}(X(p))$ as:

$$
\operatorname{Var}(X(p))=\beta^{0}+\beta^{1} p+\beta^{2} p^{2} .
$$

With this variance function, we can capture any monotone price-variance relation as well as non-monotone relations with one maximum or minimum point. In order to estimate the coefficients in equation (3.6) we use the sample variance $\operatorname{Var}\left(X\left(\rho_{i}\right)\right)$. First we need to calculate the variance of sample demand for each $\rho_{i} \in \mathbf{p}$, but for some $\rho_{i}$ we do not have enough number of observations to come up with the variance. Hence, we calculate the sample variance for $\rho_{i}$ if there are at least 5 observations with that price, and if there are less than 5 observations the variance is found by a linear interpolation of the available variances. Remember from Section 3.1 that we just consider products with at least 5 prices where we can calculate the variance. In this way we come up with $\operatorname{Var}\left(X\left(\rho_{i}\right)\right)$ for all $i=1, \cdots, 10$. Then, we write $\operatorname{Var}\left(X\left(\rho_{i}\right)\right)$ as a price dependent function:

$$
\operatorname{Var}\left(X\left(\rho_{i}\right)\right)=\beta^{0}+\beta^{1} \rho_{i}+\beta^{2} \rho_{i}^{2}+\omega_{i} .
$$

Again with least squares method we estimate the coefficients of the regression equation (3.7). When we apply this procedure the resulting variance functions for products in Figure 3.4 are illustrated in Figure 3.6.

We do not estimate a specific function in order to find out the mean demand, and we directly use the average sample demand for each $\rho_{i}$ where there are at least 5 observations and the others are linearly interpolated. At the end, we come up with a variance, $\operatorname{Var}\left(X\left(\rho_{i}\right)\right)$ and mean $E\left(X\left(\rho_{i}\right)\right)$ for each price $\rho_{i}$. We will use this model as a benchmark for evaluating the potential profit improvements of using a model which is more general than the additive and the multiplicative models.

Up to this point we modelled demand in three different ways and estimated the parameters of these models for each product. We evaluated the additive and the multiplicative models using statistical tests focusing on the correct representation of the variance. In the rest of this chapter we apply the 

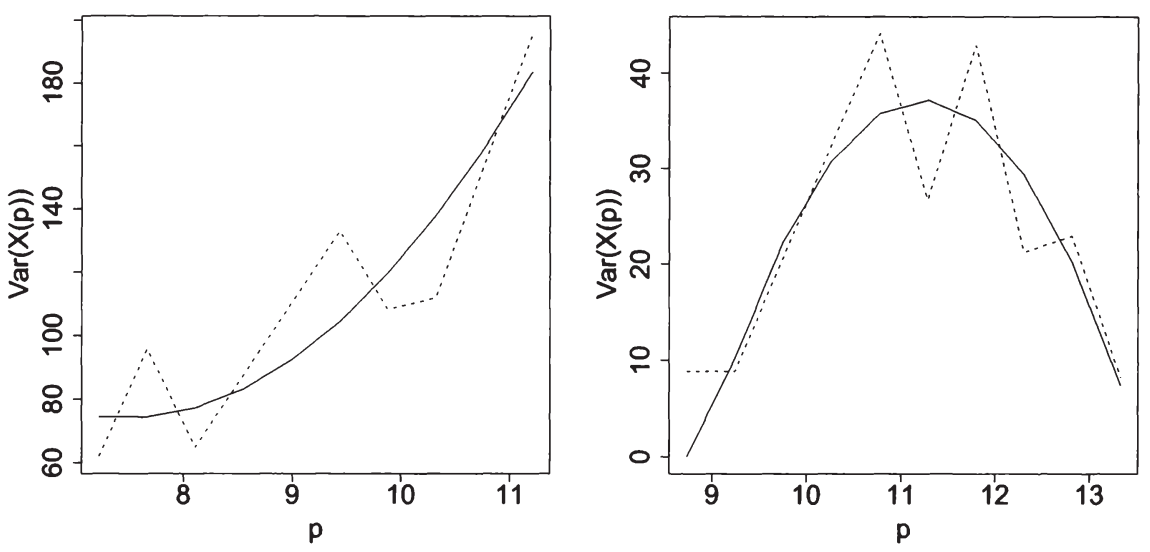

Figure 3.6: Sample variance (dashed) and fitted variance (solid) of two example products

price-setting newsvendor model for each product using the three demand models. We evaluate and compare the optimal policies resulting from each model, and using the empirical observations we simulate the policies to calculate and compare what we call the real average profits.

\subsection{Simulation of profits}

In this section we find the optimal price and inventory levels under different demand models using the newsvendor formula. Thus we need to calculate the expected profits which require specification of the cost parameters. Since we do not have access to the information about the real cost structure, we made some assumptions: 1) the unit purchasing cost is set as $80 \%$ of the smallest observed price, i.e. $\left.c=0.80 \rho_{1}, 2\right)$ there is no salvage value for leftover inventory and no penalty cost for unsatisfied demand.

In order to calculate expected profits we need the distribution function of demand. Hence, we should specify a price dependent distribution function $F(p, x)$ corresponding to each of the three demand models.

For the additive and the multiplicative models we need the distribution of the error terms $U^{A}$ and $U^{M}$ (see equations 2.3 and 2.4) to derive the demand distribution. The first step is to estimate the distribution of the residuals $\hat{u}$ and $\hat{\nu}$ from the linear and the log-linear models. While it is theoretically possible to find the best fitting distribution separately for each 
Table 3.5: Summary of the demand distributions under the three models

\begin{tabular}{llll}
\hline Model & Distribution & Mean & Variance \\
\hline Additive & Normal & $\hat{a}+\hat{b} p$ & $\operatorname{Var}\left(U^{A}\right)$ \\
Multiplicative & Log-normal & $\hat{m} p^{\hat{n}} E\left(U^{M}\right)$ & $\hat{m} p^{\hat{n}} \operatorname{Var}\left(U^{M}\right)$ \\
General & Normal & Sample mean, $E(X(\rho))$ & $\beta^{0}+\beta^{1} p+\beta^{2} p^{2}$ \\
\hline
\end{tabular}

product and for each functional form, we assume a normal distribution both for $\hat{u}$ and $\hat{\nu}$ for all the products.

The distribution of $U^{A}$ is directly estimated as a normal distribution with the mean and the variance of $\hat{u}$ :

$$
E\left(U^{A}\right)=0 \quad \operatorname{Var}\left(U^{A}\right)=\operatorname{Var}(\hat{u})
$$

The distribution of $U^{M}$ is estimated as a log-normal distribution with the mean and variance of $\hat{\nu}$ :

$$
\begin{gathered}
E\left(U^{M}\right)=e^{\operatorname{Var}(\hat{\nu}) / 2} \\
\operatorname{Var}\left(U^{M}\right)=e^{\operatorname{Var}(\hat{\nu})}\left(e^{\operatorname{Var}(\hat{\nu})}-1\right) .
\end{gathered}
$$

Using these parameters the demand distribution under the additive and the multiplicative models are derived. The corresponding distribution functions are denoted as $F^{A}(p, x)$ and $F^{M}(p, x)$ respectively. For the general model we assume a normal distribution with the sample mean and the variance as in Equation (3.6) and the distribution function $F^{G}(p, x)$. Table 3.5 summarizes this part showing which distribution is used under each model and lists the corresponding mean and variance.

After specifying the distribution functions and the parameters, first we find the optimal inventory level for each price in the observed range, i.e. for all $\rho_{i}$, using the explicit formula in equation (2.1). For example, for the additive model optimal inventory level satisfies

$$
F^{A}\left(\rho_{i}, y^{A *}\left(\rho_{i}\right)\right)=\frac{\rho_{i}-c}{\rho_{i}}
$$

and the expected profit using the $y^{A *}\left(\rho_{i}\right)$ is

$$
\Pi^{A}\left(\rho_{i}\right)=\left(\rho_{i}-c\right)-\rho_{i} \int_{0 \text { Emel Arikan - 978-3-631 }}^{y^{A *}\left(\rho_{i}\right)} F^{A}\left(\rho_{i}, x\right) d x .
$$


Then we calculate the expected profit for each $\rho_{i}$ and find the optimal price $\bar{\rho}$ by enumerating over all $\rho_{i}$. Then, for the additive model, the optimal price and the optimal inventory level are:

$$
\begin{gathered}
\bar{\rho}^{A}=\underset{\rho_{i}}{\operatorname{argmax}}\left\{\Pi^{A}\left(\rho_{i}\right)\right\} \\
\bar{y}^{A}=y^{A *}\left(\bar{\rho}^{A}\right) .
\end{gathered}
$$

We do not exceed the observed range of prices for the search because for the unobserved prices it will not be possible to simulate the profits and make a comparison among the different policies. The drawback of limiting the prices to such a compact range is that for a number of products the optimal price of two or three of the models result in the same boundary price. For those products the simulated real profits differ only because of the different inventory policies but not the pricing policy.

By simulating the policies using the observed data we calculate the real profits which is the average of the simulated profits. Let $\bar{x}^{A}=\left\{\bar{x}_{1}^{A}, \ldots, \bar{x}_{J}^{A}\right\}$ be the vector of $J$ demand observations where the corresponding price is equal to $\rho_{A}$. For the additive model $\pi^{A}$ refers to the real profit which is calculated by:

$$
\pi^{A}=\frac{1}{J}\left(\bar{\rho}^{A}-c\right) \sum_{j} \min \left(\bar{x}_{j}^{A}, \bar{y}^{A}\right) .
$$

The corresponding real profits for the multiplicative, $\pi^{M}$, and the general model, $\pi^{G}$, are calculated accordingly. In sections 3.5.1 and 3.5.2 we compare the three models based on the real profits.

The traditional way of price and inventory optimization follows a sequential approach. First, the optimal price is set, probably by the marketing department, and then the inventory decision is made given the preset price. In order to evaluate the effectiveness of this approach, we apply a sequential optimization procedure where the optimal price is calculated based on the mean demand and the inventory level is found given the optimal price, and compare this procedure with the joint optimization. Using the sequential approach the optimal price for the additive model is denoted as $\bar{\rho}^{d A}$ where $d$ stands for deterministic and the resulting real profit is $\pi^{d A}$. We compare the joint and the sequential procedures for the additive and the multiplicative models in section 3.5.3. 

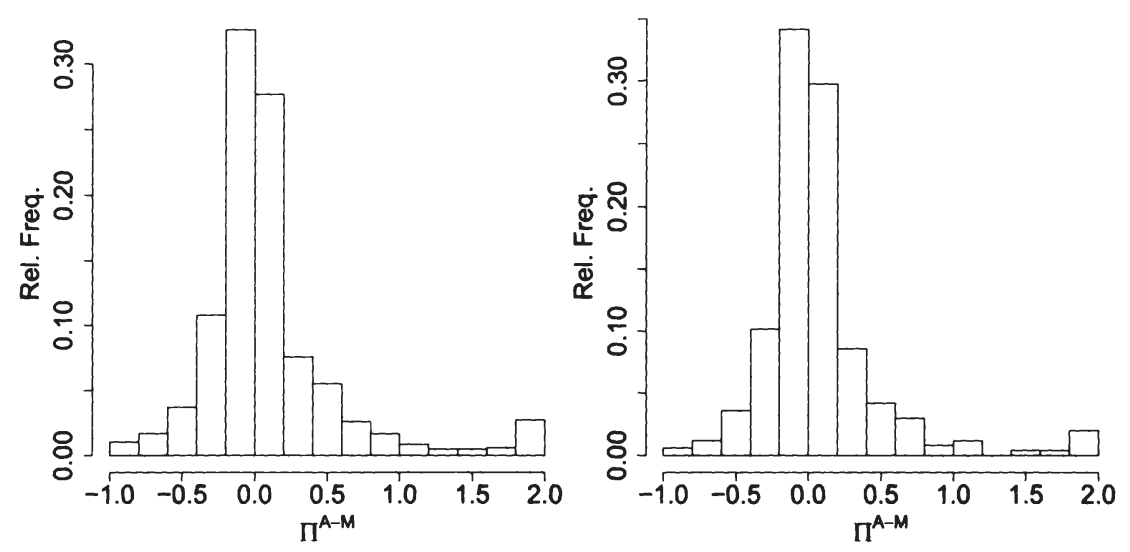

Figure 3.7: Profit improvement by using the multiplicative model instead of the additive, left for all products, right for the nac. products. The last category includes all ratios $\geq 2$.

3.5.1 Comparison of the additive and the multiplicative models based on simulated profits

In this section we compare the additive and the multiplicative models based on the real profits. Figure 3.7 shows the histogram of profit improvement by using the multiplicative model instead of the additive where

$$
\pi^{A-M}=\frac{\pi^{M}-\pi^{A}}{\pi^{A}} .
$$

Note that, in the figure, we cut off the ratio at 2 and put a mass at 2 to count for the larger values. The multiplicative model seems to perform a little bit better than the additive one since $\pi^{A-M}$ has a tendency to the positive values. When we consider all the products which satisfy the face validity conditions of both models, average $\pi^{A-M}$ is $9.5 \%$, and for nac. products it is $7.5 \%$. Thus the multiplicative model results in higher profits, but only slightly and there is a considerable part $(45.8 \%)$ of all products where the additive model gives higher profits.

Table 3.6 shows for each test the percentage of products in each type where the additive model results in a larger profit than the multiplicative one. Comparing the results with the $\mathrm{P}_{\mathrm{E}}$-test for model comparison we see a slightly better performance of the linear (log-linear) model for those products, 
Table 3.6: Percentage of products where the linear model performs better than the log-linear

\begin{tabular}{cc|ccc}
\hline Type & Conclusion & P $_{\text {E-test }}$ & $\begin{array}{c}\text { B-P test } \\
\text { all products }\end{array}$ & White test \\
\hline 1 & Linear & 54.1 & 51.7 & 50.8 \\
2 & Log-linear & 41.5 & 43.7 & 44.1 \\
3 & None & 43.4 & 44.0 & 44.9 \\
4 & Both & 46.1 & 47.1 & 46.3 \\
\hline \multicolumn{2}{r|}{} & \multicolumn{3}{|c}{ nac. products } \\
\hline 1 & Linear & 52.7 & 47.8 & 51.3 \\
2 & Log-linear & 49.0 & 43.2 & 45.6 \\
3 & None & 43.5 & 40.0 & 39.5 \\
4 & Both & 45.6 & 49.3 & 47.1 \\
\hline
\end{tabular}

where also the $\mathrm{P}_{\mathrm{E}}$-test concludes that the linear (log-linear) model is the better one. This can be observed also by comparing the simulated profits with the conclusion drawn from the two tests for homoskedasticity. While there is some improvement in real profits when we use one of the methods for model selection, the value of them is marginal. The power of using any of the methods in order to select the model which results in higher profit is not satisfactory. The probability of choosing the better model is close to $50 \%$.

The differences between the policy parameters are as expected: $60 \%$ of the time multiplicative model results in higher profits than the additive one. $30 \%$ of the time the optimal prices are the same, which might be a result of discretizing and limiting the admissible prices. Among those cases where the optimal price is the same $83 \%$ of the time optimal quantity from the additive model is larger.

\subsubsection{Comparison with the general model based on simulated profits}

When we compare the general model with the two classical models, we see a considerable increase in real profits. Figure 3.8 shows for all products, the profit improvement by using a general model instead of the additive and the multiplicative, $\pi^{A-G}$ and $\pi^{M-G}$, respectively, For the non-autocorrelated 

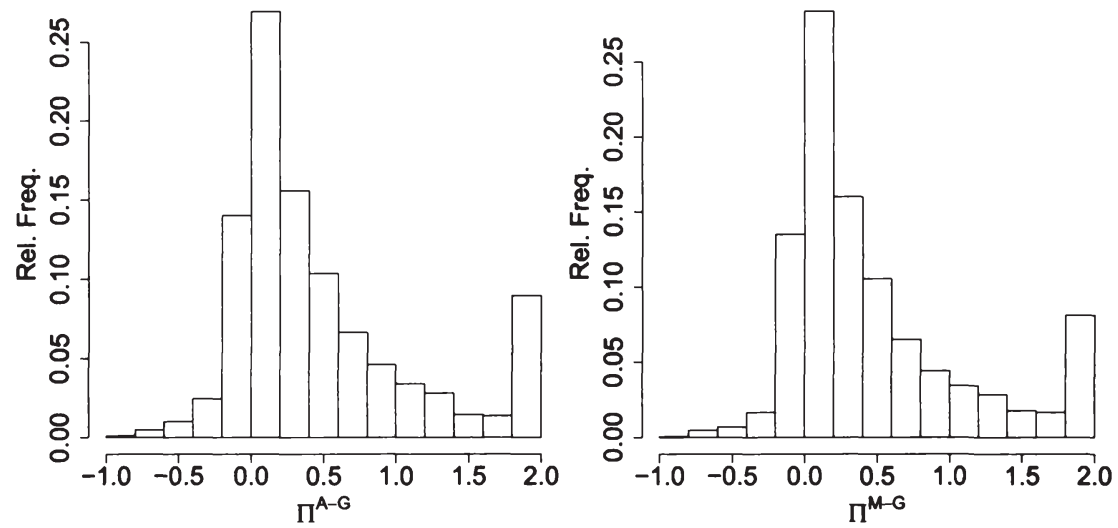

Figure 3.8: Profit improvement by using the general model instead of the additive model (left) and instead of the multiplicative model (right). Last category includes ratios $\geq 2$.

products, the picture is almost the same. $82 \%$ of $\pi^{A-G}$ and $84 \%$ of $\pi^{M-G}$ are positive which means that the general model increases the profits.

The estimated mean values of $\pi^{A-G}$ and $\pi^{M-G}$ can be found in Table 3.7 with the corresponding $95 \%$ confidence interval. There is a considerable increase in profit when the general model is used. However, it should be noted that the general model that we describe and the comparison method can have some bias. Since we do not specify a model for the mean we can exactly capture the averages in the sample, but it would not be possible to estimate demand for an unobserved price. For such a purpose we need to specify a mean function which would decrease the performance of the general model. Hence not doing comparisons based on out-of sample prices creates an advantage for the general model. However, such an out-of sample comparison would be impossible because of data requirements. We have a limited number of price observations and keeping some of them out of the estimation process would not be practical. In reality even if the numbers do not turn out to be that large as reported in Table 3.7, the results show that there is potential for improvement compared to both classical models.

An interesting issue is the differences in the optimal prices and inventory levels suggested by different models. $48 \%$ of the time optimal price under the additive model is smaller than the optimal price under the general model i.e. $\bar{\rho}^{A}<\bar{\rho}^{G}$. On the other hand, $\bar{\rho}^{M}>\bar{\rho}_{\text {Emel Arikan - } 978-3-631-75394-1}^{G}$ of the time. These numbers 
Table 3.7: Average profit improvement by using the general model (in \%)

\begin{tabular}{l|cc|cc}
\hline & \multicolumn{2}{|c|}{ All products } & \multicolumn{2}{c}{ Nac. products } \\
& Avg. & Conf. Int. & Avg. & Conf. Int. \\
\hline$\pi^{A-G}$ & 47.7 & $(45.2,50.4)$ & 46.4 & $(41.2,52.0)$ \\
$\pi^{M-G}$ & 47.8 & $(45.2,50.3)$ & 47.3 & $(42.1,52.6)$ \\
\hline
\end{tabular}

are in line with the theory which says that the multiplicative model results in higher prices. When we look at the cases where $\bar{\rho}^{A}=\bar{\rho}^{G}$, we see that $94 \%$ of the time $\bar{y}^{A}<\bar{y}^{G}$. The difference is quite considerable since on average $\bar{y}^{A}=0.74 \bar{y}^{G}$. Similarly, $92 \%$ of the cases where $\bar{\rho}^{M}=\bar{\rho}^{G}$ result in $\bar{y}^{M}<\bar{y}^{G}$, and on average $\bar{y}^{M}=0.67 \bar{y}^{G}$. Therefore, both for the additive and the multiplicative models when the price is the same as the general one, the optimal quantity is larger for the general one.

\subsubsection{Comparison of the joint and the sequential optimization based on simulated profits}

The comparison of the sequential optimization with the joint optimization, shows that there is a statistically significant improvement but the numbers are not very high. Figure 3.9 depicts the histogram of the improvement under the additive and the multiplicative demand models. For the general model it is not reasonable to calculate the optimal price in a sequential approach, since we need a function for the price dependent mean demand.

In Table 3.8 we see the confidence intervals and the average improvement. When we consider all products $4-6 \%$ profit increase can be expected by considering the stochasticity and inventory availability already for the pricing decision. However, if we combine the results from the previous section, we can say that a larger amount of improvement comes from correctly specifying

Table 3.8: Average profit improvement of using joint optimization instead of sequential optimization, under the additive and the multiplicative models (in \%)

\begin{tabular}{l|cc|cc}
\hline & \multicolumn{2}{|c|}{ All products } & \multicolumn{2}{c}{ Nac. products } \\
& Avg. & Conf. Int. & Avg. & Conf. Int. \\
\hline$\pi^{d A-A}$ & 6.2 & $(4.2,8.2)$ & 6.5 & $(2.9,10.4)$ \\
$\pi^{d M-M}$ & 4.1 & $(2.7,5.5)$ & 2.8 & $(0.3,5.6)$ \\
\hline
\end{tabular}



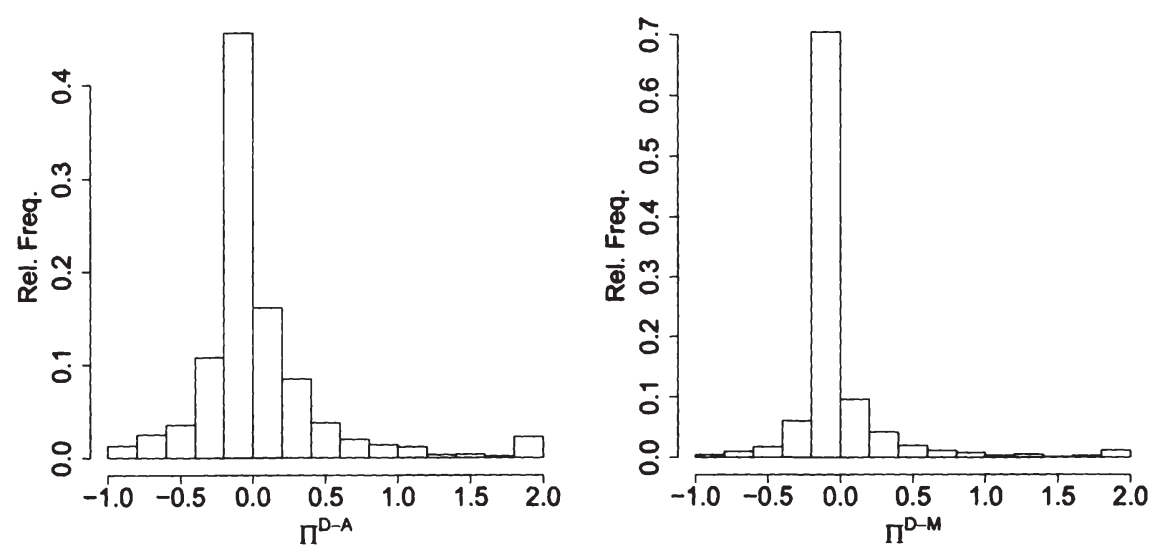

Figure 3.9: Profit improvement from joint optimization under the additive model (left) and the multiplicative model (right). The last category includes ratios $\geq 2$.

the stochastic demand process. For the additive model moving from the sequential to the joint optimization brings in $6 \%$ increase, and moving from the classical to a general demand model brings in $48 \%$ increase (see table 3.7). Overall, this would mean an increase of $59 \%$ from the sequential to the joint approach with a correctly defined demand process.

The difference in the structure of the optimal policy is exactly in line with the theory: for the multiplicative model, for all cases $\bar{\rho}^{M} \leq \bar{\rho}^{d M}$ and for the additive one just $0.6 \%$ of the time $\bar{\rho}^{A}>\bar{\rho}^{d A}$ which is probably a discretization issue.

\subsubsection{Optimal policy with a limited inventory level}

In some cases there might be a fixed amount of inventory or capacity for which the price should be optimized. The revenue management literature deals with this topic considering several aspects, and there is a considerable lot of research from the classical airline to retail revenue management. Being aware of the more complex nature of this problem and more sophisticated policies, in this section we model the problem within the newsvendor framework as a single period problem. Our aim is to compare the deterministic optimal prices with the stochastic ones and gain insights about factors that effect the optimal price. In this section we do not consider the multiplicative model. 
Since the inventory level is fixed, purchasing cost is not relevant and the profit is considered to be the same as revenue. When the inventory level is $y$ we calculate the deterministic optimal price as $\rho^{d}=\max \left(\rho_{\text {unlim }}, \rho_{\text {lim }}\right)$ where $\rho_{\text {unlim }}=\operatorname{argmax}_{\rho_{i}}\left(\rho_{i}\left(\hat{a}+\hat{b} \rho_{i}\right)\right)$ is the price which maximizes expected revenues under unlimited inventory based on mean demand, and $\rho_{\text {lim }}=$ $\min \left\{\rho_{i} \mid \hat{a}+\hat{b} \rho_{i} \leq y\right\}$ is the price which makes the expected demand equal to $y$. For each product we consider seven inventory levels such that $y=$ factor $* \rho_{\text {unlim }}$ where factor $=\{0.2,0.4,0.6,0.8,1,1.2,1.4\}$.

Our main interest in this section is to gain insights into how the optimal prices are determined. Intuitively the optimal price should be where there is no gain from increasing or decreasing price for one more unit. Let us remember how the revenues are affected by price. When the price is increased from $p_{1}$ to a larger price $p_{2}$, per unit revenue would increase, and if we can still sell the same amount as we sold under $p_{2}$ revenues would increase. However, the condition of selling the same amount is not very realistic. Obviously increasing price will decrease expected demand and consequently expected sales. Hence, the increasing per unit revenue and decreasing sales should satisfy some balance at the optimal price. In order to investigate this effect further, we suggest measuring the change in expected sales by increasing price for each inventory level as

$$
\varepsilon\left(\rho_{i}, y\right)=-\frac{S\left(\rho_{i}, y\right)-S\left(\rho_{i+1}, y\right)}{S\left(\rho_{i}, y\right)} \frac{\rho_{i}}{\rho_{i}-\rho_{i+1}}
$$

where $S\left(\rho_{i}, y\right)$ is the expected sales given $\rho_{i}$ and $y . S\left(\rho_{i}, y\right)$ is calculated using the normal distribution resulting from the additive model. $\varepsilon\left(\rho_{i}, y\right)$ measures the percentage change in expected sales by the percentage change in price, so it is the price elasticity of expected sales. When we start with a price $\rho_{i}$ and increase it to $\rho_{i+1}$ if the percentage decrease in expected sales is larger than the percentage increase in price, the loss is larger than the gain and $\rho_{i+1}$ can not be the optimal price. On the other hand, if we decrease the price to $\rho_{i-1}$ and the percentage increase in sales is smaller than the decrease in price $\rho_{i-1}$ can not be optimal. Therefore $\rho_{i}$ should give the local maximum and the change in expected sales and the change in price should be equal at $\rho_{i}$, i.e. $\varepsilon\left(\rho_{i}, y\right)=1$ at the optimal price. We know that for the additive model the optimal price is unique, so $\varepsilon\left(\rho_{i}, y\right)=1$ should be satisfied at only one price. That would mean $\varepsilon\left(\rho_{i}, y\right)$ is probably monotone increasing or decreasing in price. Let us take this discussion as our hypothesis, and try to investigate it through the observations. 
Table 3.9: Price difference between the additive stochastic and deterministic models with limited inventory

\begin{tabular}{l|rrrrrrr}
\hline Factor & 0.2 & 0.4 & 0.6 & 0.8 & 1 & 1.2 & 1.4 \\
\hline$\rho^{d}>\rho^{A}$ & 37.0 & 47.9 & 41.8 & 0 & 0 & 0 & 0 \\
$\rho^{d}<\rho^{A}$ & 0 & 0 & 0.2 & 29.3 & 41.5 & 33.0 & 21.1 \\
\hline
\end{tabular}

We want to check if $\varepsilon\left(\rho^{A}, y\right)=1$ for all quantities and products. However, since we use a discrete number of prices it is not realistic to expect that $\varepsilon\left(\rho^{A}, y\right)=1$ exactly. Hence, we compare it with the elasticity of the neighbor prices in the grid. Let $\rho^{A}=\rho_{i}$, then we have two important observations:

$$
\begin{gathered}
\varepsilon\left(\rho_{i-1}, y\right) \leq \varepsilon\left(\rho_{i}, y\right) \leq \varepsilon\left(\rho_{i+1}, y\right), \\
\varepsilon\left(\rho_{i-1}, y\right)<1<\varepsilon\left(\rho_{i+1}, y\right)
\end{gathered}
$$

for all the products and all the inventory levels. (3.8) means that the elasticity of sales is increasing in price at least around the optimal price, and when we look at the whole price range, we see that this continues to holds for each price, so for the additive model $\varepsilon(\rho, y)$ is monotone increasing in price for all inventory levels $y$ that we considered. Moreover, from (3.9) we conclude that $\varepsilon(\rho, y)$ is close to 1 at the optimal price.

When we consider the general model, the observations are not that exact as for the additive one. The first inequality in (3.8) is satisfied in all cases, but the second one is satisfied in only $70 \%$ of the time. Similarly, (3.9) is also not satisfied for all the cases. Hence, we can conclude that the sales elasticity is not necessarily increasing in price for the general model. However, since we do build the general model with quite loose assumptions, e.g. we do not have any assumptions on the mean. The structure of the results under this model might not be very smooth. Therefore we try to investigate the monotonicity property by fitting a linear function on the elasticities with respect to price and evaluate the slope of this function statistically. We see that $92-98 \%$ of the cases where price is significant end up with a positive price coefficient, i.e. $\varepsilon(p, y)$ increases in price.

Now we can compare the optimal prices in the light of the above discussion. Table 3.9 shows the percentage of products where the deterministic price is larger than the stochastic one and vice versa among 3223 products. For a large number of products the two prices turn out to be the same, for which we should consider the effect of limited number of feasible prices. The sales 


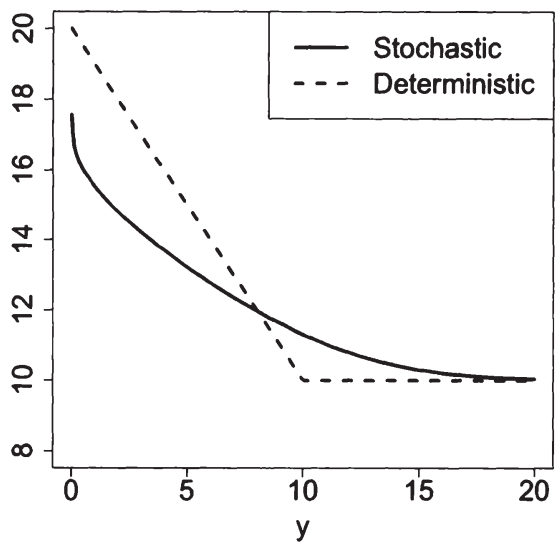

Figure 3.10: Optimal price for the stochastic and deterministic models with limited inventory

elasticity evaluated at the deterministic price explains the differences in prices. Whenever $\rho^{d}>\rho^{A}, \varepsilon\left(\rho^{d}, y\right)>1$ so at the deterministic price $\varepsilon$ is greater than 1 and in order to decrease it to 1 price should be decreased since $\varepsilon$ is monotone in price. The similar argument on the other direction holds for the cases $\rho^{d}<\rho^{A}$.

When the price and inventory are jointly optimized it is well known that the stochastic optimal price is smaller than the deterministic one (see Section 2.2.3). However, we see that when the inventory level is set in advance, the relation depends on the inventory level. We can observe a structure on the number of products in each category with respect to $y$. For small inventory deterministic price is larger than the stochastic one, and for large inventory levels it is the other way around. For most of the products the prices look like as depicted in Figure 3.10.

Such a structure is not observed when we look at the difference in optimal price between the additive and the general model in Table 3.10. As inventory increases the percentage of both relations increases, which means the number of cases where the two prices are equal is decreasing in $y$. However, there is no structure with respect to the direction of the change.

Lastly, Table 3.11 shows the profit improvements by using the additive stochastic model instead of the deterministic one $\pi^{d-A}$ and using the general model instead of the additive $\pi^{A-G}$. The values are not very different than the ones in Tables 3.7 and 3.8 without the inventory limitation, While the 
Table 3.10: Price difference between the additive and the general models with limited inventory

\begin{tabular}{l|rrrrrrr}
\hline Factor & 0.2 & 0.4 & 0.6 & 0.8 & 1 & 1.2 & 1.4 \\
\hline$\rho^{A}>\rho^{G}$ & 9.5 & 12.9 & 15.0 & 16.7 & 18.4 & 19.5 & 20.4 \\
$\rho^{G}<\rho^{A}$ & 29.5 & 35.8 & 41.3 & 43.7 & 46.9 & 49.1 & 50.3 \\
\hline
\end{tabular}

Table 3.11: Average profit improvement of using joint optimization instead of sequential optimization under the additive model with limited inventory (in \%)

\begin{tabular}{l|rrrrrrr}
\hline \multicolumn{7}{c}{ All products } \\
\hline Factor & 0.2 & 0.4 & 0.6 & 0.8 & 1 & 1.2 & 1.4 \\
\hline$\pi^{d-A}$ & 2.6 & 2.9 & 1.8 & 1.4 & 8.0 & 7.5 & 6.7 \\
$\pi^{A-G}$ & 4.6 & 9.3 & 15.5 & 28.2 & 40.1 & 47.7 & 52.5 \\
\hline \hline \multicolumn{7}{c}{ Nac products } \\
\hline Factor & 0.2 & 0.4 & 0.6 & 0.8 & 1 & 1.2 & 1.4 \\
\hline$\pi^{d-A}$ & 0.4 & 0.6 & 0.5 & 1.2 & 4.1 & 2.0 & 1.2 \\
$\pi^{A-G}$ & 1.8 & 6.3 & 11.6 & 18.5 & 24.1 & 29.3 & 33.2 \\
\hline
\end{tabular}

improvement from the deterministic to the stochastic policy is not very high, moving from the additive to the general model has a big impact. As inventory level increases, i.e. factor increases, the improvement seems to increase too. Increasing inventory might mean less restriction and more opportunity for profit improvement by changing prices.

We can summarize the findings in this chapter as follows:

1. Selection between the additive and the multiplicative models is not trivial and the selection based on some statistical criteria does not guarantee higher profits. (Table 3.6)

2. Using a more flexible model removes the selection problem and increases profit. (Table 3.7)

3. Optimal prices can be found using sales elasticity, and the sales elasticity is increasing in price for most of the products. (Section 3.5.4) 
The first two observations motivate the analytical study of the price-setting newsvendor problem with a general demand definition, and the last one supports the main assumption made for the analysis of the model in the following Chapter 4. 


\section{Chapter 4}

\section{Analysis of the Generalized Model}

As we discussed in Chapter 2 using the additive and/or the multiplicative models imply restrictions on the demand-price relation, especially with respect to the effects of price on demand variability. Moreover, the empirical study in Chapter 3 shows that there is a big potential for improvement by using a more general model. In this chapter we consider the analytical treatment of the price-setting newsvendor problem under such a general demand definition.

While the literature is dominated by the additive and the multiplicative uncertainty models, there is a small number of papers in which different demand models are analyzed under different approaches. First in Section 4.1 we give a summary of these papers and then in Section 4.2, we present our approach for modelling demand-price relation. Here, we introduce the formal definition of sales elasticity that we intuitively describe in Section 3.5.4. Next, we present analytical results about the optimality conditions and structural properties, and the assumptions leading to these results. A numerical study follows where the results are illustrated and some of the assumptions are relaxed.

\subsection{Literature review}

The closest model formulation to ours is used by Polatoglu (1991), who studies a model without any assumptions on the structure of the demandprice relation and the inclusion of uncertainty. The distribution function of random demand $X(p)$ is defined as a general price dependent function $F(p, x)$. Existence and uniqueness of the optimal policy constitute the focus of the study. However, especially for the uniqueness problem, the conclusions are not very informative and there are no qualitative discussions about the assumptions and the results. Moreover, the effects of changing parameters and sensitivity analysis are not provided. Their main interest is the analysis of the single period problem in order to build the basis for multi period problems (which is studied in Polatoglu and Sahin (2000)), and that's why the single period problem is not studied in more detail. 
Kocabiyıkoğlu and Popescu (2005) present a deeper analysis of the problem with a general demand model. As in the classical setting they describe the random demand as a combination of a deterministic and a random part. In addition to the additive and multiplicative forms, the model is also applicable for more general structures such as $X(p)=\log (U-b p)$. The main assumption is the strict concavity of the revenue function in price i.e. $2 X_{p}(p)+p X_{p p}(p)<0$ for any risk realization $u$. This assumption allows them to easily show the uniqueness of an optimal price but at the same time restricts the analysis to a smaller group of demand definitions. Another shortcoming of their model is that it should be possible to separate the deterministic and the random part of demand. Even if there is no restriction with respect to the form of the demand function, the random variable $U$ and its distribution should not be affected by price. Functions like $X(p)=\log (U-b p)$ still imply the separability of price and the error term. Nevertheless, the model allows combinations other than the additive and the multiplicative ones.

The key to their analysis is the concept of lost sales elasticity:

$$
\kappa(p, y)=\frac{p F_{p}(p, y)}{1-F(p, y)} .
$$

When the inventory level is $y$, probability of lost sales is $1-F(p, y)$, and $\kappa(p, y)$ measures the sensitivity of the probability of lost sales with respect to price. The monotonicity of price and order quantity depend on the monotonicity of $\kappa(p, y)$. If $\kappa(p, y)$ is increasing in $p$ and $y$ optimal order quantity $y^{*}(p)$ is decreasing in price and $p^{*}(y)$ is decreasing in $y$. The uniqueness of a joint optimal solution is also guaranteed by the monotonicity of $\kappa(p, y)$.

Raz and Porteus (2006) approach the problem from a different perspective. Instead of working directly with the distribution functions, they formulate a function for some fractiles of the distribution. Figure 4.1 shows the demand distribution for different prices when $F(p, x)=1-e^{\left(-0.01 p^{2} x\right)}$. Figure 4.2, on the other hand, is the inverse distribution as a function of price for different fractiles $\alpha$.

Raz and Porteus (2006) estimate the inverse distribution function (e.g. the curves in 4.2) for a number of fractiles, which means the discretization of the distribution. In the main part of their paper, they estimate these functions for only a number of prices and use a linear approximation in between the prices. The focus is mainly on the solution procedure. For each of the linear pieces the optimal price and quantity combination is 


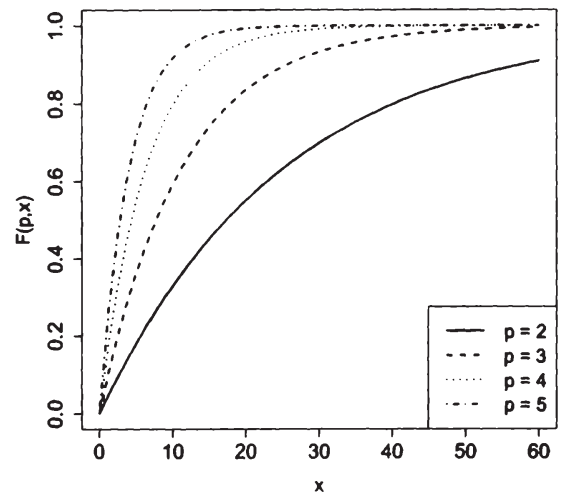

Figure 4.1: Exponential distribution function, $F(p, x)=1-e^{\left(-0.01 p^{2} x\right)}$

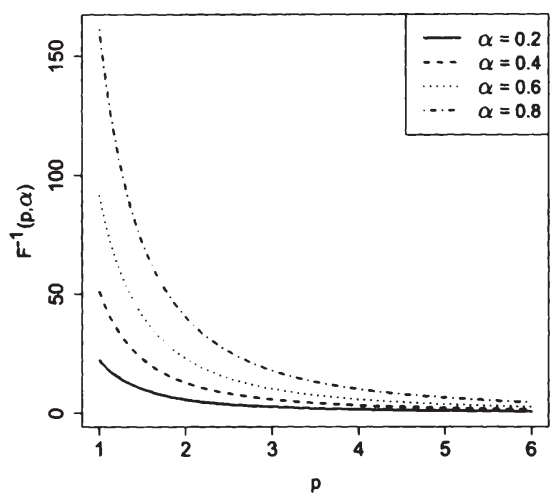

Figure 4.2: Exponential inverse distr. fun., $F^{-1}(p, \alpha)=-\ln (1-\alpha) / p^{2}$

uniquely found and, for the overall problem, the solution from each piece is enumerated. Complete enumeration turns out to be necessary because of multiple local maximums. Their model allows any demand-price relation with different variability patterns, but the results depend mainly on their specific estimation and approximation procedure.

\subsection{Model description}

Instead of using the classical approach we integrate price as a parameter of the demand distribution. This is more similar to the traditional inventory models without pricing decision. We are now dealing directly with the distribution of demand and not the distribution of the error term, $U$.

In order to characterize $X(p)$ under the classical methods, an appropriate deterministic function of price and an error term distribution $\Phi(u)$ must be specified. Specification of $\Phi(u)$ depends on the deterministic part of demand and in order to avoid negative demand it has to be bounded. On the other hand, in our case a direct approach is used and the question is to specify the distribution of demand which is observable to the decision maker.

We assume that the expected demand $E[X(p)]$ is decreasing in price. Note that $E[X(p)]$ can be interpreted as the deterministic part $d(p)$ of the classical demand definition. However, we do not require that the demand can be separated into a price dependent deterministic term and a price independent 
random term. In this sense our definition is compareable to that of Polatoglu (1991) and Polatoglu and Sahin (2000).

We assume that, as price increases probability of low demand increases and consequently expected demand decreases.

Assumption 1. $F(p, x)$ is increasing in $p$, which implies $F\left(p_{1}, x\right) \geq F\left(p_{2}, x\right)$ and $E\left[X\left(p_{1}\right)\right] \leq E\left[X\left(p_{2}\right)\right]$ for $p_{1} \geq p_{2}$, i. e. $X(p)$ are ordered with respect to $p$ in the sense of first order stochastic dominance.

When the prices are in a continuous range, Assumption 1 implies $F_{p}(p, x) \geq$ 0 , for all $x \geq 0$. Both the additive and the multiplicative models satisfy this assumption.

The important step to the analysis of the newsvendor profit is the analysis of the components that create the profit. Even if the demand model attracts the most attention in the analysis, what leads to profits is the expected sales. Hence, it is essential to understand the interaction of the decision variables, i.e. price and inventory, not only with demand but also with sales.

When demand is considered as a pure deterministic function of price, one of the most important aspects of demand is its sensitivity to price changes. Price elasticity of demand is the measure for the responsiveness of demand to price changes. While studying the profit function, price elasticity of demand is the determinant factor since sales and demand are the same.

When demand is assumed to be stochastic, there appear two complications. First, the elasticity of demand is not easy to define since we do not directly have a function of price which returns the demand, but we have a function that gives probability of observing different levels of demand. Second, the responsiveness of sales comes into question in addition to demand, which might not have one-to-one correspondence, especially because sales is affected also by the inventory level.

Before moving on to the specific optimization problem we present the two basic concepts that are helpful for the understanding of these interactions. First we describe what the failure rate of random demand means and how it is related to price and inventory levels. Then we analyze the elasticity of sales with respect to price and inventory, where we need some properties of the failure rate. 


\subsubsection{Failure rate and failure rate ordering}

One of the main properties of a distribution function that we use in our analysis is the failure rate. Failure rate (hazard rate) of the random demand $X(p)$ can be defined as:

$$
h(p, x)=\frac{f(p, x)}{1-F(p, x)} .
$$

The terminology comes from reliability theory (Barlow and Proschan, 1996). If we modify the explanation given by Porteus (2002) (pg.134) in order to fit it to our context, the failure rate of a demand distribution can be interpreted as the conditional probability that the demand will be at most $x+d x$ units given that it is at least $x$ units. The demand distribution is said to be IFR (increasing failure rate) if $\frac{\partial}{\partial x} h(p, x)>0$ for all $x$. Uniform, Normal, Exponential distributions are IFR while Gamma, Beta, and Weibull distributions are IFR with some restrictions on their parameters.

The generalized failure rate is:

$$
g(p, x)=\frac{x f(p, x)}{1-F(p, x)}
$$

If $\frac{\partial}{\partial x} g(p, x)>0$ for all $x$, the distribution is IGFR (increasing generalized failure rate). An IFR distribution is clearly also IGFR, but the opposite does not always hold. Especially in the pricing and revenue management literature, it is common to assume IGFR distributions (see Ziya et al. (2004) and Lariviere (2006)).

If the stocking quantity is $y$, the failure rate $h(p, y)$ and the generalized failure rate $g(p, y)$ at stocking quantity is described by Lariviere and Porteus (2001) as follows:

The failure rate gives (roughly) the percentage decrease in the probability of a stock out from increasing the stocking quantity by one unit, the generalized failure rate gives (roughly) the percentage decrease in the probability of a stock out from increasing the stocking quantity by $1 \%$.

Thus, the two functions can be interpreted as a measure for the sensitivity of the probability of lost sales with respect to the inventory level. Specifically, the generalized failure rate, $g(p, y)$, is the elasticity of $(1-F(p, y))$, i.e. the probability of lost sales, with respect to $y$. 
IFR and IGFR properties are related to the effect of changes in demand $x$ for a constant price $p$. On the other hand, the effect of price changes leads to the failure rate ordering of the random demand. $X\left(p_{1}\right)$ is said to be larger than $X\left(p_{2}\right)$ with respect to failure rate order if $h\left(p_{1}, x\right) \geq h\left(p_{2}, x\right)$ for $p_{1} \geq p_{2}$ (see e.g. Müller and Stoyan (2002), Shaked and Shanthikumar (2007)). If the failure rate of $X(p)$ increases as $p$ increases, we call that $X(p)$ has failure rate ordering with respect to $p$, i.e. $h\left(p_{1}, x\right) \geq h\left(p_{2}, x\right)$ for all $p_{1} \geq p_{2}$. For a continuous range of prices this means, $\frac{\partial}{\partial p} h(p, x)>0$ i.e. $h(p, x)$ increases in $p$. If $h(p, x)$ is increasing in $p, g(p, x)$ is also increasing in $p$.

Failure rate ordering is a stronger concept than the first order stochastic dominance described in Assumption 1 since the former implies the latter one. While we use the first order stochastic dominance (Ass. 1) as a general assumption throughout the analyses, some of the results depend on failure rate ordering. Whenever this is the case we mention it explicitly.

\subsubsection{Elasticity of expected sales}

The key to the analysis of the profit function is the analysis of the expected sales with respect to price and the inventory level. Expected sales for price $p$ and inventory level $y$ can be defined as:

$$
S(p, y)=\int_{0}^{y}[1-F(p, x)] d x
$$

The sensitivity of $S(p, y)$ with respect to $p$ and $y$ determines the structure of the optimal policy. Hence we define two measures representing this sensitivity:

1. $\varepsilon^{p}(p, y)$ is the price elasticity of expected sales:

$$
\varepsilon^{p}(p, y)=\frac{-p S_{p}(p, y)}{S(p, y)}
$$

where

$$
S_{p}(p, y)=\frac{\partial S(p, y)}{\partial p}=-\int_{0}^{y} F_{p}(p, x) d x
$$

2. $\varepsilon^{y}(p, y)$ is the inventory elasticity of expected sales:

$$
\varepsilon^{y}(p, y)=\frac{y S_{y}(p, y)}{S_{(}\left(p_{i} y_{\text {mikan }}\right. \text { - 978-3-631-75394-1 }}
$$


where

$$
S_{y}(p, y)=\frac{\partial S(p, y)}{\partial y}=1-F(p, y)
$$

Expected sales $S(p, y)$ increases as the inventory level $y$ increases, so the inventory elasticity of sales, $\varepsilon^{y}(p, y)$, is a measure for the degree of this increase in expected sales. On the other hand increasing price results in a smaller number of expected sales, and the price elasticity of sales, $\varepsilon^{p}(p, y)$, measures the sensitivity of the decrease in expected sales with respect to an increase in price. Both values are the absolute level of sensitivity hence they are both positive.

The definition of price elasticity $\varepsilon^{p}(p, y)$ does not require the ordering of demand with respect to price in the sense of first order stochastic dominance but the second order is necessary. First order stochastic dominance results in $F_{p}(p, x) \geq 0$ in our context. On the other hand, the second order stochastic dominance implies

$$
\int_{0}^{y} F_{p}(p, x) d x \geq 0
$$

The lost-sales elasticity, as defined by Kocabıyıkoğlu and Popescu (2005), requires first order stochastic dominance, because otherwise $F_{p}(p, x)$ and consequently the lost-sales elasticity might become negative for some $p$ and/or $x$.

The expected profit can be written in terms of expected sales as,

$$
\Pi(p, y)=p S(p, y)-c y .
$$

For the analysis of the expected profit with respect to price, the key is the structure of $\varepsilon^{p}(p, y)$. The following assumption, which can be supported by empirical evidence, makes it possible to come up with satisfactory results.

Assumption 2. Price elasticity of sales, $\varepsilon^{p}(p, y)$, increases in price.

Remember that for the additive model Assumption 2 is satisfied for all the products and inventory levels that we considered in the relevant part of the empirical study in Section 3.5.4, and for the general model there is statistical evidence that it is satisfied for more than $90 \%$ of the products and inventory levels.

On the other hand, inventory elasticity can be directly related to the properties of the demand distribution. Proposition 1 describes the relation between the quantity elasticity and the generalized failure rate of demand. 
Proposition 1. If demand has increasing generalized failure rate, inventory elasticity of sales, $\varepsilon^{y}(p, y)$, decreases in the inventory level $y$.

Proof. Let us write $\varepsilon^{y}(p, y)$ in terms of the demand distribution as,

$$
\varepsilon^{y}(p, y)=\frac{y[1-F(p, y)]}{\int_{0}^{y}[1-F(p, x)] d x} .
$$

The nominator can be decomposed into two parts as follows,

$$
\begin{aligned}
y[1-F(p, y)] & =\int_{y}^{\infty} y f(p, x) d x \\
& =\int_{0}^{y}[1-F(p, x)] d x-\int_{0}^{y} x f(p, x) d x
\end{aligned}
$$

so that we can write,

$$
\begin{aligned}
\varepsilon^{y}(p, y) & =1-\frac{\int_{0}^{y} x f(p, x) d x}{\int_{0}^{y}[1-F(p, x)] d x} \\
& =1-\frac{\int_{0}^{y} g(p, x)[1-F(p, x)] d x}{\int_{0}^{y}[1-F(p, x)] d x} .
\end{aligned}
$$

When we differentiate $\varepsilon^{y}(p, y)$ with respect to $y$ by using Leibniz rule, we conclude that it is decreasing in $y$.

Some of the results that we will present in the following sections depend on the failure rate ordering of demand with respect to price. Proposition 2 shows the relation of failure rate ordering to the two elasticity concepts that we described.

Proposition 2. If the failure rate of demand increases in price, i.e. failure rate ordering with respect to price,

1. the price elasticity of sales, $\varepsilon^{p}(p, y)$, increases in inventory level $y$,

2. the quantity elasticity of sales, $\varepsilon^{y}(p, y)$, decreases in price $p$.

Proof. If the failure rate increases in price,

$$
\frac{\partial h(p, x)}{\partial p}=\frac{F_{x p}(p, x)[1-F(p, x)]+F_{p}(p, x) F_{x}(p, x)}{[1-F(p, x)]_{\text {Arikan - 978-3-631-75394-1 }}^{2}} \geq 0
$$


implying

$$
F_{x p}(p, x)[1-F(p, x)]+F_{p}(p, x) F_{x}(p, x) \geq 0 .
$$

Let us define $\kappa(p, x)$ as,

$$
\kappa(p, x)=\frac{p F_{p}(p, x)}{1-F(p, x)} .
$$

The first derivative of $\kappa(p, x)$ with respect to $x$ is,

$$
\frac{\partial \kappa(p, x)}{\partial x}=p \frac{F_{x p}(p, x)[1-F(p, x)]+F_{p}(p, x) F_{x}(p, x)}{[1-F(p, x)]^{2}} \geq 0
$$

so $\kappa(p, x)$ increases in $x$.

Now, we can reformulate $\varepsilon^{p}(p, y)$ in terms of $\kappa(p, x)$ :

$$
\begin{aligned}
\varepsilon^{p}(p, y) & =\frac{\int_{0}^{y} p F_{p}(p, x) d x}{\int_{0}^{y}[1-F(p, x)] d x} \\
& =\frac{\int_{0}^{y} \kappa(p, x)[1-F(p, x)] d x}{\int_{0}^{y}[1-F(p, x)] d x}
\end{aligned}
$$

When we differentiate $\varepsilon^{p}(p, y)$ with respect to $y$ by using Leibniz rule, we conclude that it is increasing in $y$.

For the second part, we use the relation between the first derivative of the quantity elasticity and the price elasticity. From the first part of the proposition the following equation is positive:

$$
\frac{\partial \varepsilon^{p}(p, y)}{\partial y}=-p \frac{S_{p y}(p, y) S(p, y)-S_{y}(p, y) S_{p}(p, y)}{S^{2}}
$$

and this implies,

$$
\frac{\partial \varepsilon^{y}(p, y)}{\partial p}=y \frac{S_{p y}(p, y) S(p, y)-S_{y}(p, y) S_{p}(p, y)}{S^{2}} \leq 0 .
$$

Hence, $\varepsilon^{y}(p, y)$ decreases in price.

In Figure 4.3 and Figure 4.4 we illustrate the concepts and relations described in this section. We see that $S(p, y)$ is convex decreasing in $p$ (Fig. 4.3) and concave increasing in $y$ (Fig. 4.4). This fact is reflected as an increasing $\varepsilon^{p}(p, y)$ in $p$ and decreasing $\varepsilon^{y}(p, y)$ in $y$ satisfying the conditions described in Assumption 2 and Proposition 1. Note that the implications on 

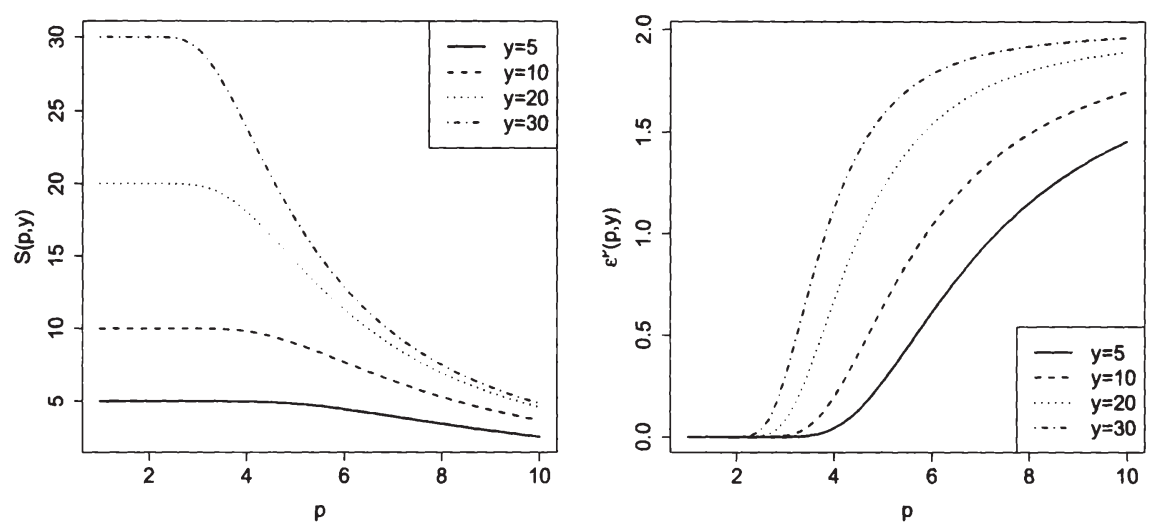

Figure 4.3: $S(p, y)$ and $\varepsilon^{p}(p, y)$ under Gamma distribution with shape $=50 p^{-2}$, scale $=10$

the absolute change in $S(p, y)$ are the same: the absolute change in $S(p, y)$ is decreasing in both $p$ and $y$. As $p$ increases $S(p, y)$ might possibly converge to zero and as $y$ increases it converges to the mean demand. The relations defined in Proposition 2 is observed on the ordering of $\varepsilon^{p}(p, y)$ with respect to $y$ and $\varepsilon^{y}(p, y)$ with respect to $p$.

In some of the further analyses we use the failure rate ordering as condition, but the effect of failure rate ordering is through its effect on the elasticities. Hence, the same results can be achieved by the corresponding condition on $\varepsilon^{p}(p, y)$ and/or $\varepsilon^{y}(p, y)$.

\subsection{Non-integrated approach}

In this section we consider the two decisions - pricing and inventory control - separately. We call this setting the non-integrated approach since one of the decisions is assumed to be made in advance, independent of the second decision, or because of some exogenous restrictions the decision maker is not able to control both decisions. Other than the relevance to some problem settings, the analyses in this section are necessary for the analysis of the joint optimization under the integrated approach.

First, in Section 4.3.1, we study the setting where the newsvendor does not decide on price e.g. price is set by the market or set by the marketing department before ordering decision. The difference to the classical price- 

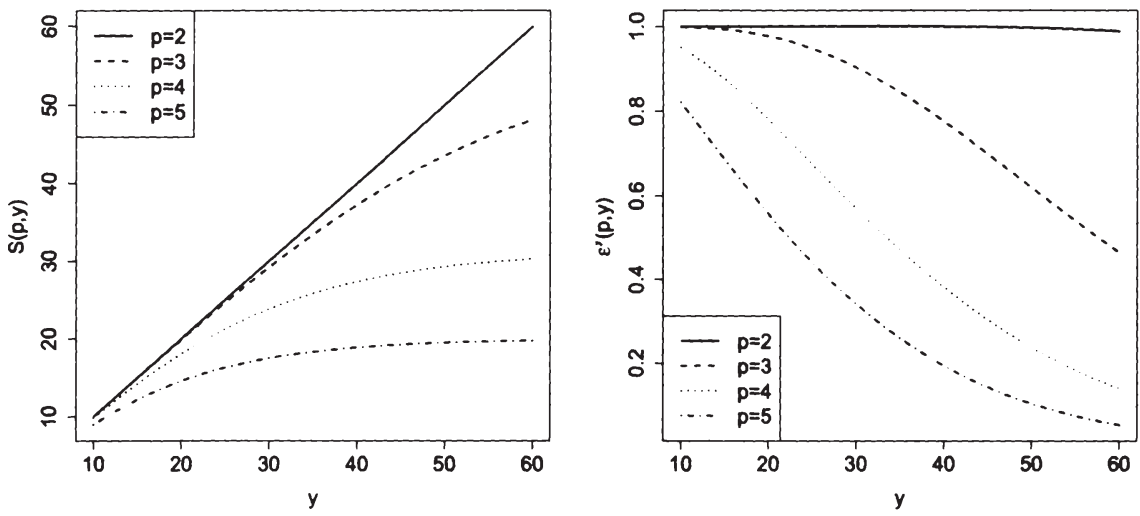

Figure 4.4: $S(p, y)$ and $\varepsilon^{y}(p, y)$ under Gamma distribution with shape $=50 p^{-2}$, scale $=10$

taking newsvendor problem described in Section 2.1 is the explicit treatment of the effect of price on demand. On the other hand, in Section 4.3.2 we analyze solely the pricing problem assuming the inventory level is fixed. A typical situation where the problem is relevant can be the sales periods in the retailing industry. At the end of the season, a discounted price is charged for the remaining inventory. Hence, the price depends on the inventory level and no further replenishment is possible.

\subsubsection{Optimizing order quantity}

When the price is not a decision variable but an exogenous variable the optimality results of the price-taking newsvendor model continue to hold, but the effect of price changes on the optimal ordering quantity becomes more complicated.

Theorem 1. For any given price $p$ expected profit $\Pi(p, y)$ is concave in $y$ and the optimum ordering quantity $y^{*}(p)$ is:

$$
y^{*}(p)=F^{-1}(p, C r(p)) .
$$

where $C r(p)=(p-c) / p$ is the critical fractile.

Proof. The second derivative of $\Pi(p, y)$ with respect to $y$ is negative, i.e.

$$
\Pi_{y y}(p, y)=-p f(p, y)<0 .
$$


and the first order condition gives (4.6).

If the leftover inventory can be sold for a salvage value of $v(v<c)$ and/or if the lost sales cost a shortage penalty of $b(b \geq 0)$, Theorem 1 continues to hold but the critical fractile should be changed as $\operatorname{Cr}(p)=(p-c+b) /(p-v+b)$.

Recall that in the price-taking newsvendor model, the optimal order quantity increases as $\mathrm{Cr}$ increases, since the profitability of the product increases. However, if the profitability of the product increases because of an increase in price, it is not clear if the ordering quantity should really increase. Since the price-taking newsvendor model assumes a price-independent demand distribution, the increase in price does not affect the amount demanded but affects only the fractile, and this leads to an increase in the optimum ordering quantity. However, the economic intuition tells that an increase in price might lead to a decrease in the overall demand, which is captured by the stochastic ordering described in Assumption 1 in our model. In that case, the increasing profitability and the decreasing demand counteract with each other and the resulting change in optimum order quantity depends on the interplay of the two opposite affects.

Contrary to the findings from classical economic theory and also from recent price-setting newsvendor models it can be shown that for important cases the optimal ordering quantity is not a decreasing function over the whole range of admissible prices, but is increasing for small selling prices and decreasing for higher prices.

In Theorem 2 we give a general result concerning the monotonicity of the optimal ordering quantity.

Theorem 2. The optimal ordering quantity $y^{*}(p)$ is increasing in $p$ if

$$
\frac{d}{d p} C r(p) \geq F_{p}\left(p, y^{*}(p)\right)
$$

and is decreasing otherwise.

Proof. Optimality of $y^{*}(p)$ implies $C r(p)=F\left(p, y^{*}(p)\right)$ (see (4.6)). By the implicit function theorem

$$
\begin{aligned}
& \frac{d}{d p} C r(p)=\frac{c}{p^{2}}=F_{p}\left(p, y^{*}(p)\right)+F_{x}\left(p, y^{*}(p)\right) \frac{d y^{*}(p)}{d p}, \\
& \frac{d y^{*}(p)}{d p}=\frac{\frac{c}{p^{2}}-F_{p}\left(p, y^{*}(p)\right)}{F_{x}\left(p, y_{\text {m }}^{*}(p)_{\text {Arikan }}\right.} \text {. }
\end{aligned}
$$




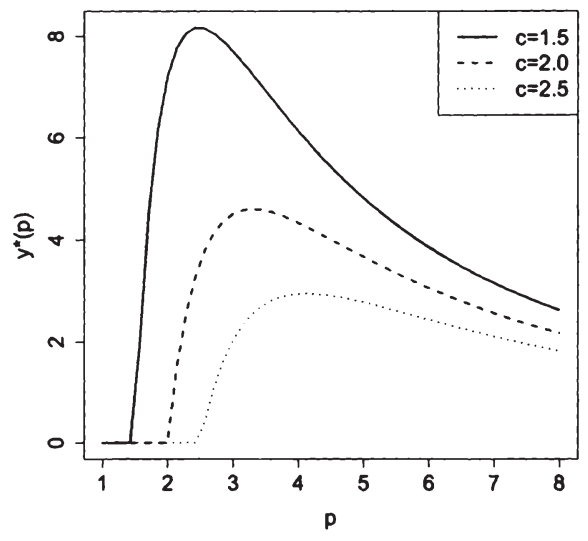

Figure 4.5: Optimal order quantity $y^{*}(p)$ for $F(p, x)=1-e^{-\left(0.01 p^{2} x\right)}$

Since $F_{x}\left(p, y^{*}(p)\right)=f\left(p, y^{*}(p)\right) \geq 0$,

$$
\frac{d y^{*}(p)}{d p} \geq 0 \Leftrightarrow \frac{c}{p^{2}}=\frac{d}{d p} C r(p) \geq F_{p}\left(p, y^{*}(p)\right) .
$$

$d C r(p) / d p \leq F_{p}\left(p, y^{*}(p)\right)$ is equivalent to the condition $\kappa\left(p, y^{*}(p)\right) \geq 1$ used by Kocabiyıkoğlu and Popescu (2005).

Figure 4.5 shows $y^{*}(p)$ as a function of $p$ for different costs. As price increases, the profitability $\operatorname{Cr}(p)=(p-c) / p$ and consequently the cycle service level of the product is increasing which implies higher order quantities. However, at the same time, there is a decrease in demand so a lower inventory level should be considered. The relative strength of these two effects defines the behavior of the optimal order quantity. As can be seen in Figure 4.5 the price range where optimal order quantity increases is not necessarily a small, negligible range. When $c=2$ the increase continues up to $p=p^{o}=3.3$ which is $65 \%$ larger than cost. For $c<p<p^{\circ}$, the increase in profit because of increasing profitability dominates the influence of decreasing demand. For $p^{\circ}<p$, the influence of the decreasing demand dominates increasing profitability, which implies decreasing $y^{*}(p)$.

With respect to the effect of cost parameters, the results of the price-taking newsvendor model continues to hold, but for the sake of completeness we repeat them in Theorem 3 . 
Theorem 3. The optimal order quantity is decreasing in cost $c$, increasing in salvage value $v$ and shortage penalty $\operatorname{cost} b$.

Proof. Since $F(p, x)$ is a strictly increasing distribution function, $y^{*}(p)=$ $F^{-1}(p, C r(p))$ increases as $C r(p)$ increases where

$$
C r(p)=\frac{p-c+b}{p-v+b}
$$

The conclusion follows since $\mathrm{Cr}(p)$ decreases in $c$ and increases in $v$ and $b$.

\subsubsection{Optimizing price}

In this section we analyze the pricing problem for a given inventory level. The purchasing cost of the inventory can be considered as sunk cost and the objective is to maximize revenues. Hence the analysis is the same as analyzing the expected revenue function, $R(p, y)$ where

$$
R(p, y)=p \int_{0}^{y}[1-F(p, x)] d x .
$$

When the expected revenue function is shifted down by cost, we get the expected profit function. The first derivative of expected revenue with respect to price $R_{p}(p, y)$ is the same as the first derivative of the expected profit $\Pi_{p}(p, y)$

$$
R_{p}(p, y)=\Pi_{p}(p, y)=\int_{0}^{y}\left[1-F(p, x)-p F_{p}(p, x)\right] d x .
$$

Let $p^{*}(y)$ be the price where the first derivative is equal to zero, i.e.

$$
\Pi_{p}\left(p^{*}(y), y\right)=\int_{0}^{y}\left[1-F\left(p^{*}(y), x\right)-p^{*}(y) F_{p}\left(p^{*}(y), y\right)\right]=0 .
$$

In order to guarantee that $p^{*}(y)$ is unique and that it is a maximum, the second derivative should be smaller than zero

$$
\Pi_{p p}\left(p^{*}(y), y\right)=-\int_{0}^{y}\left[2 F_{p}\left(p^{*}(y), x\right)+\underset{\text { Emel Arikan - 978-3-631-753 }}{\left.p^{*}(y) F_{p p}\left(p^{*}(y), y\right)\right] \leq 0 .}\right.
$$


The question is which properties of the demand distribution are necessary in order to fulfill the conditions in (4.9) and (4.10).

The trivial answer is: if $F(p, x)$ is convex increasing in $p$ both conditions are fullfilled. If $F(p, x)$ is convex increasing in $p$, both $F_{p}(p, x)$ and $F_{p p}(p, x)$ are positive, and $\Pi_{p p}(p, y)$ is smaller than zero for all $p$. This implies that $F(p, x)$ is increasing with increasing increments. However, if random demand is not bounded from above or if it is bounded but the upper bound does not depend on price, $F(p, x)$ can not be convex for all prices at least for large $x$. Hence, the price and/or demand should be limited.

Theorem 4 describes a weaker condition for the uniqueness of the optimal solution and provides the condition for the monotonicity of $p^{*}(y)$ in $y$.

Theorem 4. If the price elasticity of sales, $\varepsilon^{p}(p, y)$, increases in price,

1. $\Pi(p, y)$ is unimodal in $p$ so the solution to $\Pi_{p}(p, y)$ is the unique optimal price and $p^{*}(y)$ satisfies $\varepsilon^{p}\left(p^{*}(y), y\right)=1$.

2. If, additionally, $\varepsilon^{p}(p, y)$, increases in inventory level, then $p^{*}(y)$ decreases in $y$.

Proof. We can write $\Pi_{p}(p, y)$ as,

$$
\Pi_{p}(p, y)=\left(1-\varepsilon^{p}(p, y)\right) \int_{0}^{y}[1-F(p, x)] d x .
$$

Since $1-F(p, x)$ is always positive, if $\varepsilon^{p}(p, y)$ is increasing in $p, \Pi_{p}(p, y)$ can become zero at most at one point and that point corresponds to $p^{*}(y)$ such that $\varepsilon^{p}\left(p^{*}(y), y\right)=1$. This completes the first part.

For the second part, first we write,

$$
\begin{aligned}
& \frac{\partial}{\partial y} \Pi_{p}\left(p^{*}(y), y\right)= \\
& {\left[-\varepsilon_{p}^{p}\left(p^{*}(y), y\right) \frac{d p^{*}(y)}{d y}-\varepsilon_{y}^{p}\left(p^{*}(y), y\right)\right] \int_{0}^{y}\left[1-F\left(p^{*}(y), x\right)\right] d x=0 .}
\end{aligned}
$$

If we arrange the terms,

$$
\frac{d p^{*}(y)}{d y}=-\frac{\varepsilon_{y}^{p}\left(p^{*}(y), y\right)}{\varepsilon_{p}^{p}\left(\underline{p}_{n}^{*}(y)_{\mathcal{A}} y\right)} .
$$


Since $\varepsilon^{p}(p, y)$ increases in both $p$ and $y$,

$$
\frac{d p^{*}(y)}{d y} \leq 0
$$

A sufficient condition for $\varepsilon^{p}(p, y)$ being increasing in inventory level $y$ is the failure rate ordering with respect to price (see Proposition 2). Hence failure rate ordering guarantees the monotonicity of $p^{*}(y)$. In the following theorem, Theorem 5 , we provide a different analysis of the monotonicity of $p^{*}(y)$ under failure rate ordering.

Lemma 1. Let $\xi(p, x)=1-F(p, x)-p F_{p}(p, x)$. If the failure rate of demand $h(p, x)$ increases in price,

1. $\xi(p, x)$ has at most one root with respect to $x$ for any given $p$ such that $\xi\left(p, x^{0}(p)\right)=0$, and $\xi_{x}\left(p, x^{0}(p)\right)<0$, implying crossing zero from above,

2. if there exists a finite optimal price $p^{*}(y)$ then $\xi\left(p^{*}(y), y\right)<0$.

Proof. We can write $\xi(p, x)$ as,

$$
\xi(p, x)=(1-F(p, x))(1-\kappa(p, x)) .
$$

From Proposition 2 we know that $\kappa(p, x)$ increases in $x$ if $h(p, x)$ increases in price. If for a specific price $p, \xi\left(p, x^{0}(p)\right)=0$ for some finite $x^{0}(p)$ then $\kappa\left(p, x^{0}(p)\right)=1$ and since $\kappa(p, x)$ increases there can not be any other $x$ that makes $\xi(p, x)$ equal to zero. Hence, for all $x \geq x^{0}(p), \kappa(p, x) \geq 1$ and $\xi(p, x) \leq 0$. This completes the first part.

For the second part, consider the following,

$$
\Pi_{p}\left(p^{*}(y), y\right)=\int_{0}^{y} \xi\left(p^{*}(y), x\right) d x=0 .
$$

From the first part we conclude that $\xi\left(p^{*}(y), x\right)$ is either positive for all $x \leq y$ or it is first positive and then negative. Since $\Pi_{p}\left(p^{*}(y), y\right)=0$ it can not be always positive and we can write it as,

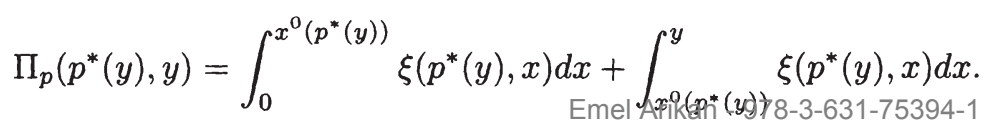


Within the borders of the first integral $\xi\left(p^{*}(y), x\right)$ is always positive and in the second integral it is always negative, which means $\xi\left(p^{*}(y), y\right)<0$

Theorem 5. If $h(p, x)$ increases in $p$, the optimal price $p^{*}(y)$ decreases in the inventory level $y$.

Proof.

$$
\frac{\partial}{\partial y} \Pi_{p}\left(p^{*}(y), y\right)=\xi\left(p^{*}(y), y\right)+\frac{d}{d y} p^{*}(y) \Pi_{p p}\left(p^{*}(y), y\right)=0
$$

and

$$
\frac{d}{d y} p^{*}(y)=-\frac{\xi\left(p^{*}(y), y\right)}{\Pi_{p p}\left(p^{*}(y), y\right)} .
$$

$\Pi_{p p}\left(p^{*}(y), y\right) \leq 0$ since, by definition, $p^{*}(y)$ is the unique maximizer. If $h(p, x)$ increases in $p$ then $\xi\left(p^{*}(y), y\right)<0$ from Lemma 1 , and hence,

$$
\frac{d}{d y} p^{*}(y) \leq 0 .
$$

Remark 1. Theorem 4 depends on $\varepsilon^{p}(p, y)$ being increasing in $y$ for all $p$. Similarly, Theorem 5 employs the failure rate ordering over the whole range of $p$ and $y$, i.e. $h_{p}(p, x) \geq 0$ for all $p$ and $x$. However, as long as a unique maximizer exists, for the monotonicity of $p^{*}(y)$ it is enough if $\varepsilon^{p}(p, y)$ is increasing in $y$ when $p=p^{*}(y)$ and/or $h_{p}(p, x) \geq 0$ for all $x \leq y$ evaluated at $p=p^{*}(y)$.

As described in Theorem 5 and Remark 1 failure rate ordering over the whole or a specific range of $p$ and $x$ is a sufficient condition for the monotonicity of $p^{*}(y)$.

On the other hand, if failure rate does not depend on price the optimal price does not change with inventory level. For example if $F(p, x)=1-$ $e^{\left(-\lambda\left(\ln (x)+p^{b}\right)\right)}$ which can also be represented in a separated way as $X(p)=$ $e^{\left(U-p^{b}\right)}$ where $U$ has an exponential distribution with a mean of $1 / \lambda$, failure rate of $F(p, x)$ is $\lambda / x$. This means, $\kappa(p, x)$ is constant in $x$, and the optimal price for any inventory level $y$ is equal to $p^{*}$ such that $\kappa\left(p^{*}, x\right)=1$. Hence the optimal price for the joint optimization is the same as the optimal price calculated for any inventory level and hence can be computed easily.

For the price optimization sometimes the problem is how to ensure a finite optimal price, i.e. a finite price which makes (4.9) hold. For example if the 
mean demand is represented with the function $a p^{-1}$ it is optimal to increase price as much as possible so $p^{*}(y)=\infty$. Checking $p^{*}(y)$ where $y=\infty$, can help find out if such a problem is existing for the specific models. Since $p^{*}(y)$ decreases in $y, p^{*}(\infty)$ is a lower bound for $p^{*}(y)$, and if $p^{*}(\infty)=\infty$ optimal price for any inventory level is also infinite. When the inventory level is infinite, sales is equal to demand and the resulting expected revenue is not constrained by inventory, i.e. $R(p, \infty)=p E[X(p)]$ and if this unconstrained revenue is increasing in price then the expected revenue for any inventory level is also increasing in price. Note that we use increasing in the weak sense. If $F(p, x)=1-e^{-(a p) x}$, the unconstrained revenue is independent of price: if there is infinite inventory, whatever $p$ is, the expected revenue is $a$, so weakly increasing in price. Since the unconstrained revenue is increasing, for any finite inventory level expected revenue strictly increases in price and converges to $a$.

Since the optimal price for a given inventory level is determined just by the revenue function, it is not affected by changes in the ordering or production cost. However, if there is any salvage value or penalty cost, optimal price is affected by them.

Theorem 6. The optimal price increases if there is positive salvage value and/or penalty cost.

Proof. Let salvage value be $v \geq 0$ and penalty cost be $b \geq 0$, then the expected profit function is:

$$
\Pi(p, y)=(p-c+b) y-b E[X(p)]-(p-v+b) \int_{0}^{y} F(p, x) d x
$$

and the first derivative with respect to price is

$$
\Pi_{p}(p, y)=\int_{0}^{y} \xi(p, x) d x+\int_{0}^{y} v F_{p}(p, x) d x+\int_{y}^{\infty} b F_{p}(p, x) d x
$$

If $p^{*}(y)$ is the optimal price without salvage value or penalty cost the first integral in (4.13) is zero. The following two integrals are positive, and $\Pi_{p}(p, y)$ is positive which means the profit is still in an increasing phase, so the price should be further increased to reach the optimum.

When price increases, the amount of expected leftovers increases but if the leftover inventory can be sold out for a salvage value, price increase becomes less harmful in terms of its effect on leftovers. Likewise, penalty cost makes the lost sales more important. Without the penalty cost, i.e. $b=\overline{\bar{A}}$ 0 0 , the only 
cost of lost sales is the opportunity cost. When $b>0$, in order to decrease the amount of lost sales, price should be increased so that demand decreases.

Note that if there is salvage cost i.e. $v \leq 0$, and $b=0$ optimal price should decrease since leftovers are more costly. If $b \geq 0$ and $v \leq 0$ the effect depends on the relative size of expected lost sales and expected leftovers as well as the difference between $b$ and $v$. Since the leftovers and the lost sales depend on the inventory level, the effect might be different for different inventory levels. For small $y$ lost sales are more probable and for larger $y$ leftovers become more likely. Hence, for small $y$ price should be increased while for larger $y$ it should be decreased.

\subsection{Integrated approach}

In this section we analyze the problem where both the price and the order quantity are decision variables. The objective is again maximizing expected profit, but now the price and the order quantity should be set simultaneously. In Section 4.4.1 we deal with the uniqueness of optimal policy and its relation to the sales elasticity. We employ the common way of analysis on the optimal price or quantity path. In Section 4.4.2 we discuss some structural properties of the optimal policy with respect to the monotonicity of optimal quantity in price and the effect of cost on the policy parameters.

\subsubsection{Optimality conditions}

In order to check if the joint optimization results in a unique solution, we can follow analysis on either of the optimal paths, i.e. $\Pi\left(p^{*}(y), y\right)$ or $\Pi\left(p, y^{*}(p)\right)$. The first of these two functions take the optimal price function, $p^{*}(y)$, as an input and the resulting problem is finding the optimal order quantity $\bar{y}$, through this optimal price path. $\bar{y}$ is then the optimal quantity for the joint optimization problem and the corresponding optimal price is $\bar{p}=p^{*}(\bar{y})$. Similarly, the second function is a function on the optimal quantity path where the problem is finding the optimal price, $\bar{p}$, and the corresponding optimal order quantity $\bar{y}=y^{*}(\bar{p})$.

The uniqueness of the solution on one of the optimal paths implies the uniqueness of the optimal price-quantity combination for the joint optimization. The following two theorems show that as long as the properties of the sales elasticity continues to hold at least on one of the optimal paths, uniqueness can be ensured. 
Theorem 7. If on the optimal price path the inventory elasticity of sales, $\varepsilon^{y}\left(p^{*}(y), y\right)$, decreases in the inventory level $y$, the expected profit $\Pi\left(p^{*}(y), y\right)$ is concave in $y$.

Proof. In order to prove concavity, we will show that the second derivative of profit on the optimal price path is negative, i.e.

$$
\frac{\partial^{2}}{\partial y^{2}} \Pi\left(p^{*}(y), y\right) \leq 0
$$

where

$$
\begin{aligned}
\frac{\partial^{2}}{\partial y^{2}} \Pi\left(p^{*}(y), y\right)= & p^{\prime}(y) S_{y}\left(p^{*}(y), y\right)+p^{*}(y) p^{\prime}(y) S_{p y}\left(p^{*}(y), y\right) \\
& +p^{*}(y) S_{y y}\left(p^{*}(y), y\right)
\end{aligned}
$$

and

$$
p^{\prime}(y)=\frac{d}{d y} p^{*}(y)
$$

In the next equations of this proof we will drop the arguments for tractability. $S$ and all its derivatives have the argument $\left(p^{*}(y), y\right)$, e.g. $S=S\left(p^{*}(y), y\right)$ and $S_{p y}=S_{p y}\left(p^{*}(y), y\right)$. Moreover, $p^{*}=p^{*}(y)$ and $p^{\prime}=p^{\prime}(y)$. Now we can write (4.14) as

$$
\frac{\partial^{2}}{\partial y^{2}} \Pi\left(p^{*}(y), y\right)=p^{\prime} S_{y}+p^{*} p^{\prime} S_{p y}+p^{*} S_{y y}
$$

We can write the first derivative of $\varepsilon^{y}\left(p^{*}(y), y\right)$ with respect to $y$ as follows:

$$
\frac{\partial}{\partial y} \varepsilon^{y}\left(p^{*}(y), y\right)=\frac{1}{S^{2}} y S\left(p^{*} p^{\prime} S_{p y}+p^{\prime} S_{y}+p^{*} S_{y y}\right)+p^{*} S_{y}\left(S-y S_{y}\right)
$$

If $\varepsilon^{y}\left(p^{*}(y), y\right)$ is decreasing in $y, \frac{\partial}{\partial y} \varepsilon^{y}\left(p^{*}(y), y\right) \leq 0$ and, hence,

$$
y S\left(p^{*} p^{\prime} S_{p y}+p^{\prime} S_{y}+p^{*} S_{y y}\right)+p^{*} S_{y}\left(S-y S_{y}\right) \leq 0 .
$$

$S-y S_{y}$ is always positive since,

$$
\int_{0}^{y}(1-F(p, x)) d x \geq y[1-F(p, y)] \text { for all } y \text { and } p .
$$


Since $p^{*}$ and $S_{y}$ are also positive, in order to satisfy (4.17), the following should hold:

$$
y S\left(p^{*} p^{\prime} S_{p y}+p^{\prime} S_{y}+p^{*} S_{y y}\right) \leq 0
$$

and

$$
\begin{aligned}
\frac{\partial^{2}}{\partial y^{2}} \Pi\left(p^{*}(y), y\right)= & p^{\prime} S_{y}+p^{*} p^{\prime} S_{p y}+p^{*} S_{y y} \\
& \leq \frac{-p^{*} S_{y}\left(S-y S_{y}\right)}{y S} \leq 0
\end{aligned}
$$

As shown in Proposition 1 if demand is IGFR $\varepsilon^{y}(p, y)$ is increasing in $y$ for any $p$. Hence the condition in Theorem 7 requires that this property continues to hold on the optimal price path. The next Theorem 8 looks at the problem on the optimal quantity path.

Theorem 8. If on the optimal quantity path the price elasticity of sales, $\varepsilon^{p}\left(p, y^{*}(p)\right)$, increases in price $p$, the expected profit $\Pi\left(p, y^{*}(p)\right)$ is unimodal in price.

Proof. For unimodality, the second derivative of $\Pi\left(p, y^{*}(p)\right)$ with respect to $p$ should be negative for prices where the first derivative is equal to zero. Let us define $\bar{p}$ as the price(s) at which the following equality holds:

$$
\Pi_{p}\left(\bar{p}, y^{*}(\bar{p})\right)=S\left(\bar{p}, y^{*}(\bar{p})\right)+\bar{p} S_{p}\left(\bar{p}, y^{*}(\bar{p})\right)=0 .
$$

We want to ensure that $\bar{p}$ is unique by showing,

$$
\left.\frac{\partial^{2}}{\partial p^{2}} \Pi\left(p, y^{*}(p)\right)\right|_{p=\bar{p}} \leq 0 .
$$

We will again drop the arguments of $S$ and its derivatives, such that in the next equations of this proof, the arguments of $S$ and its derivatives are always $\left(\bar{p}, y^{*}(\bar{p})\right)$, and,

$$
y^{\prime}=\left.\frac{d}{d p} y^{*}(p)\right|_{p=\bar{p}} .
$$

Now we can write

$$
\left.\frac{\partial^{2}}{\partial p^{2}} \Pi\left(p, y^{*}(p)\right)\right|_{p=\bar{p}}=S_{p}+y^{\prime} S_{y}+S_{p}+\bar{p} S_{p p}+\bar{p} y^{\prime} S_{p y},
$$


and

$$
\left.\frac{\partial}{\partial p} \varepsilon^{p}\left(p, y^{*}(p)\right)\right|_{p=\bar{p}}=\frac{-1}{S^{2}}\left(\left(S_{p}+p S_{p p}+\bar{p} y^{\prime} S_{p y}\right) S-\left(S_{p}+y^{\prime} S_{y}\right) p S_{p}\right) .
$$

If $\varepsilon^{p}\left(p, y^{*}(p)\right)$ increases in $p$,

$$
\left(S_{p}+\bar{p} S_{p p}+\bar{p} y^{\prime} S_{p y}\right) S-\left(S_{p}+y^{\prime} S_{y}\right) \bar{p} S_{p} \leq 0 .
$$

Since $S\left(\bar{p}, y^{*}(\bar{p})\right)=-\bar{p} S_{p}\left(\bar{p}, y^{*}(\bar{p})\right)$ we can replace $S$ by $-\bar{p} S_{p}$ in (4.18) and,

$$
-\bar{p} S_{p}\left(S_{p}+\bar{p} S_{p p}+\bar{p} y^{\prime} S_{p y}+S_{p}+y^{\prime} S_{y}\right) \leq 0 .
$$

Since $S_{p}$ is always negative,

$$
\left.\frac{\partial^{2}}{\partial p^{2}} \Pi\left(p, y^{*}(p)\right)\right|_{p=\bar{p}}=S_{p}+\bar{p} S_{p p}+\bar{p} y^{\prime} S_{p y}+S_{p}+y^{\prime} S_{y} \leq 0 .
$$

\subsubsection{Structural properties}

From Theorem 2 we conclude that if the pricing and inventory decisions are not made simultaneously $y^{*}(p)$ is increasing for small prices and decreasing for larger ones. Next result shows that if $X(p)$ has failure rate ordering, the combination of $\bar{y}$ and $\bar{p}$ results in a point at the decreasing range of $y^{*}(p)$.

Theorem 9. If $h(p, x)$ increases in $p$, optimal order quantity for the joint optimization, $\bar{y}$, is in the decreasing part of $y^{*}(p)$, i.e.

$$
\left.\frac{d}{d p} y^{*}(p)\right|_{p=\bar{p}} \leq 0
$$

Proof. From Lemma $1 \xi\left(p^{*}(y), y\right)<0$, so $1-F\left(p, y^{*}(p)\right) \leq p F_{p}\left(p, y^{*}(p)\right)$. Since $F\left(p, y^{*}(p)\right)=C r(p)=(p-c) / p$,

$$
\frac{c}{p^{2}} \leq F_{p}\left(p, y^{*}(p)\right)
$$

and from Theorem 2 optimal order quantity is decreasing in price.

Figure 4.6 illustrates Theorem 9. The circles on the curves indicate the respective $\bar{p}$ and $\bar{y}$ combinations for three different cost parameters. Since the 


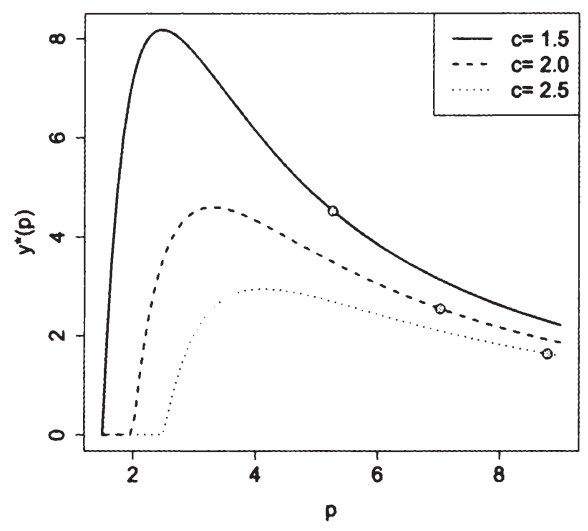

Figure 4.6: Optimal order quantity $y^{*}(p)$ for $F(p, x)=1-e^{-\left(0.01 p^{2} x\right)}$. Circles mark $(\bar{p}, \bar{y})$ combinations

demand distribution implies failure rate ordering, Theorem 9 is relevant and all these circles turn out to be on the decreasing parts of the corresponding curves.

Our last result on the joint optimization problem is the relation of the optimal parameters, $\bar{p}$ and $\bar{y}$, with the per unit ordering cost $c$.

Theorem 10. When per unit ordering/production cost $c$ increases,

1. optimal quantity $\bar{y}$ decreases,

2. and if $h(p, x)$ increases in $p$, optimal price $\bar{p}$ increases.

Proof. For the first part we need to show that

$$
\frac{\partial}{\partial c} \Pi_{y}\left(p^{*}(y), y\right) \leq 0
$$

where,

$$
\left.\Pi_{y}\left(p^{*}(y), y\right)=\left(p^{*}(y)-c\right)-p^{*}(y) F\left(p^{*}(y), y\right)\right)
$$

Since $p^{*}(y)$ does not depend on cost at all,

$$
\frac{\partial}{\partial c} \Pi_{y}\left(p^{*}(y), y\right) \underset{\text { Emel Arikan }}{=-c \leq 0}
$$


This completes the first part. For the second part, we need to show

$$
\frac{\partial}{\partial c} \Pi_{p}\left(p, y^{*}(p)\right) \geq 0
$$

where,

$$
\Pi_{p}\left(p, y^{*}(p)\right)=\int_{0}^{y^{*}(p)}\left(1-F(p, x)-p F_{p}(p, x)\right) d x .
$$

From Theorem 3 and Lemma 1, the result follows:

$$
\frac{\partial}{\partial c} \Pi_{p}\left(p, y^{*}(p)\right)=\frac{\partial y^{*}(p)}{\partial c}\left[1-F\left(p, y^{*}(p)\right)-p F_{p}\left(p, y^{*}(p)\right)\right] \geq 0 .
$$

\subsection{Sales elasticity for additive and multiplicative models}

Since some of our results depend on the assumption of increasing sales elasticity in price, $\varepsilon^{p}(p, y)$, in this section we discuss the generality of this assumption. Specifically we show its relation to the additive and multiplicative models.

The additive and the multiplicative models are often analyzed with respect to the optimal price and the optimal stocking factor ( e.g. Petruzzi and Dada (1999), Agrawal and Seshadri (2000), Dana and Petruzzi (2001), Monahan et al. (2004)). For the additive model stocking factor is $s=y-d(p)$ and for the multiplicative model $s=y / d(p)$. A common approach is to maximize profit in $p$ and $s$, and in return finding the corresponding $y$. Hence, the important analytical property is not the concavity (or unimodality) of $p$ for a fixed $y$, but the concavity (or unimodality) of $p$ for a fixed $s$. In Proposition 3 we show that, if $s$ is fixed, $\varepsilon^{p}(p, s)$ increases in $p$ as long as the deterministic part has increasing price elasticity.

Proposition 3. If $d(p)$ has increasing price elasticity, i.e. $\frac{-p d^{\prime}(p)}{d(p)}$ increases in $p, \varepsilon^{p}(p, s)$ increases in $p$ for

1. the additive model where $X(p)=X^{A}(p)=d(p)+U$

2. the multiplicative model where $X(p)=X^{M}(p)=d(p) U$ 
Proof. For the additive model expected sales for a given $p$ and $s$ is,

$$
S^{A}(p, s)=d(p)-\int_{s}^{\infty}(u-s) \phi(u) d u
$$

and the first derivative with respect to $p$ is

$$
S_{p}^{A}(p, s)=d^{\prime}(p)
$$

The sales elasticity and its derivative is then,

$$
\begin{gathered}
\varepsilon^{p}(p, s)=\frac{-p d^{\prime}(p)}{d(p)-\int_{s}^{\infty}(u-s) \phi(u) d u} \\
\frac{\partial}{\partial p} \varepsilon^{p}(p, s)=\frac{\left(-d^{\prime}(p)-p d^{\prime \prime}(p)\right) S^{A}(p, s)+p d^{\prime}(p)^{2}}{S^{A}(p, s)^{2}}
\end{gathered}
$$

The only part which can be negative in 4.19 is $\left(-d^{\prime}(p)-p d^{\prime \prime}(p)\right)$. If it is negative,

$$
\left(-d^{\prime}(p)-p d^{\prime \prime}(p)\right) S^{A}(p, s) \geq\left(-d^{\prime}(p)-p d^{\prime \prime}(p)\right) d(p)
$$

since $S^{A}(p, s) \leq d(p)$, and hence

$$
\left(-d^{\prime}(p)-p d^{\prime \prime}(p)\right) S^{A}(p, s)+p d^{\prime}(p)^{2} \geq\left(-d^{\prime}(p)-p d^{\prime \prime}(p)\right) d(p)+p d^{\prime}(p)^{2} \geq 0
$$

since $d(p)$ has increasing price elasticity. For the multiplicative model,

$$
\begin{gathered}
S^{M}(p, s)=d(p) \int_{0}^{s}(1-\Phi(u)) d u \\
S_{p}^{M}(p, s)=d^{\prime}(p) \int_{0}^{s}(1-\Phi(u)) d u .
\end{gathered}
$$

Hence, the expected sales elasticity is exactly the same as the elasticity of $d(p)$

$$
\varepsilon^{p}(p, s)=\frac{-p d^{\prime}(p)}{d(p)}
$$

and it is increasing in $p$.

As described in Proposition 3 , if the stocking factor is fixed, $\varepsilon^{p}(p, s)$ increases in $p$ independent of the distribution of the error term. On the other hand, if we have a certain level of inventory $y$, as the price changes 
stocking factor will also change, so, in that case, it is not possible to assume a fixed $s$. Proposition 4 describes the behavior of sales elasticity for the multiplicative model when $y$ is fixed and $s$ can change.

Proposition 4. For the multiplicative model, $\varepsilon^{p}(p, y)$ increases in price if $d(p)$ has increasing price elasticity and the error has IGFR.

Proof. $\Phi(\cdot)$ and $\phi(\cdot)$ are the cdf and pdf of $u$ respectively, and $h(u)$ is the generalized failure rate of $u$. Expected sales is,

$$
S(p, y)=d(p) \int_{0}^{y / d(p)}[1-\Phi(u)] d u
$$

and the first derivative is

$$
S_{p}(p, y)=d^{\prime}(p) \int_{0}^{y / d(p)}[1-\Phi(u)] d u-y \frac{d^{\prime}(p)}{d(p)}\left[1-\Phi\left(\frac{y}{d(p)}\right)\right] .
$$

Let $s=\frac{y}{d(p)}$ then

$$
\varepsilon^{p}(p, y)=\frac{-p S_{p}(p, y)}{S(p, y)}=\frac{-p d^{\prime}(p)}{d(p)}\left[1-s \frac{[1-\Phi(s)]}{\int_{0}^{s}[1-\Phi(u)] d u}\right] .
$$

If $d(p)$ has increasing price elasticity then, $\frac{-p d^{\prime}(p)}{d(p)}$ is increasing in price $p$, where $d^{\prime}(p)$ is the first derivative of $d(p)$ with respect to $p$. Since $s$ is increasing in $p$, to show that $\varepsilon^{p}(p, y)$ increases in $p$ it is enough to show $A(s)$ increases in $s$, where

$$
\begin{aligned}
A(s) & =1-s \frac{[1-\Phi(s)]}{\int_{0}^{s}[1-\Phi(u)] d u} \\
& =\frac{s \Phi(s)-\int_{0}^{s} \Phi(u) d u}{\int_{0}^{s}[1-\Phi(u)] d u} \\
& =\frac{\int_{0}^{s} u \phi(u) d u}{\int_{0}^{s}[1-\Phi(u)] d u} \\
& =\frac{\int_{0}^{s} h(u)[1-\Phi(u)] d u}{\int_{0}^{s}[1-\Phi(u)] d u}
\end{aligned}
$$


Note that the following holds from integration by parts:

$$
\int_{0}^{s} u \phi(u) d u=s \Phi(s)-\int_{0}^{s} \Phi(u) d u
$$

In order to show that $A(s)$ increases in $s$ we will check its first derivative:

$$
\frac{d}{d s} A(s)=\frac{[1-\Phi(s)] \int_{0}^{s}[h(s)-h(u)][1-\Phi(u)] d u}{\left(\int_{0}^{s}[1-\Phi(u)] d u\right)^{2}}
$$

Since $h$ is an increasing function $h(s)-h(u) \geq 0$ for all $u \leq s$, so $\frac{d}{d s} A(s)$ is positive and $A(s)$ is increasing in $s$ and $\varepsilon^{p}(p, y)$ increases in $p$.

As it can be seen from Proposition 3 and Proposition 4 the possibility of analyzing the problem with respect to a stocking factor instead of the absolute level of inventory simplifies the analysis considerably. However, while the two can be used interchangeably for the joint optimization, in case of a given inventory level assuming a fixed stocking factor does not help find the optimal price. Moreover, in order to be able to do the analysis on the stocking factor, it should be possible to separate the deterministic and the stochastic parts.

\subsection{Numerical study}

In this section we present some numerical examples in order to illustrate the analytical findings of the preceding sections. The analytical results depend on some assumptions, especially the uniqueness of optimal price requires the price elasticity of sales to be increasing in price, and some of the structural properties require failure rate ordering. By numerical examples we also aim to investigate problems where not all the assumptions of the preceding sections are fulfilled.

We analyze the effect of different price-variance relations on the policy parameters and the expected profits. To do so, we compute the expected profits using different distribution functions with increasing, decreasing, or constant variances in price. Additionally, we formulate the variance as a non-monotone function of price, e.g. an increasing-decreasing or decreasingincreasing function in price. We compare the resulting policy parameters and the profits against the cases where an additive or a multiplicative model is used. Moreover, we evaluate the effect of using the mean demand for calculating the optimal price which refers to the sequential optimization, 
i.e. first the optimal price is found using the mean demand and the optimal quantity is found from the price-taking newsvendor model given the optimal price.

Throughout the numerical study we use the Gamma and the Log-normal distributions since they give the flexibility of specifying the mean and the variance independently and assume nonnegative random variables. The results of the Gamma distribution is reported here but the Log-normal distribution gives qualitatively the same results, e.g. the direction of the changes are the same while absolute values might differ.

We do not consider salvage value or penalty costs, and the unit purchasing cost is $c=1$ unless otherwise stated.

The results are presented in the following two sections where Section 4.6.1 covers the analysis under the monotone variance assumption and in Section 4.6.2 the random demand is modelled with a decreasing-increasing variance.

\subsubsection{Monotone variance}

Under this setting the mean demand is formulated as $E(X(p))=500 p^{M S}$ with $M S<0$, and the variance is $\operatorname{Var}(X(p))=500 p^{V S}$. VS and $M S$ can be interpreted as the variance sensitivity and the sensitivity of mean demand with respect to price $p . \operatorname{Var}(X(p))$ is always a monotone function of $p$ such that when $V S<0, \operatorname{Var}(X(p))$ is decreasing in $p$ and increasing when $V S>0$. When $V S=0, \operatorname{Var}(X(p))$ does not depend on $p$ and we refer to this case as the additive model. On the other hand, $C v(X(p))$ is a nondecreasing function of price under any combination of $M S$ and $V S$ values. The settings where $V S=2 M S$ is considered as the multiplicative counterpart of the model with the specific $M S$ since that implies a constant Cv. Figure 4.7 shows $E(X(p)), S D(X(p))=\sqrt{\operatorname{Var}(X(p))}$, and $C v(X(p))$ for different values of $M S$ and $V S$.

Sales elasticity and failure rate Figure 4.8 shows the price elasticity of sales, $\varepsilon^{p}(p, y)$ and the failure rate, $h(p, y)$ for $M S=-2$. As we assume in Section $4.2 .2, \varepsilon^{p}(p, y)$ increases in $p$, which assures the unique optimal price (see Theorem 4). However, $h(p, y)$ is not monotone increasing in $p$, i.e. there is no failure rate ordering, and the effect of this can also be seen in $\varepsilon^{p}(p, y)$ since it is not ordered with respect to $y$.

As price increases the IFR property of the demand distribution vanishes. Depending on $V S, h(p, y)$ curves cross each other at a specific price. This is the price where the shape parameter of the Gamma distribution becomes 

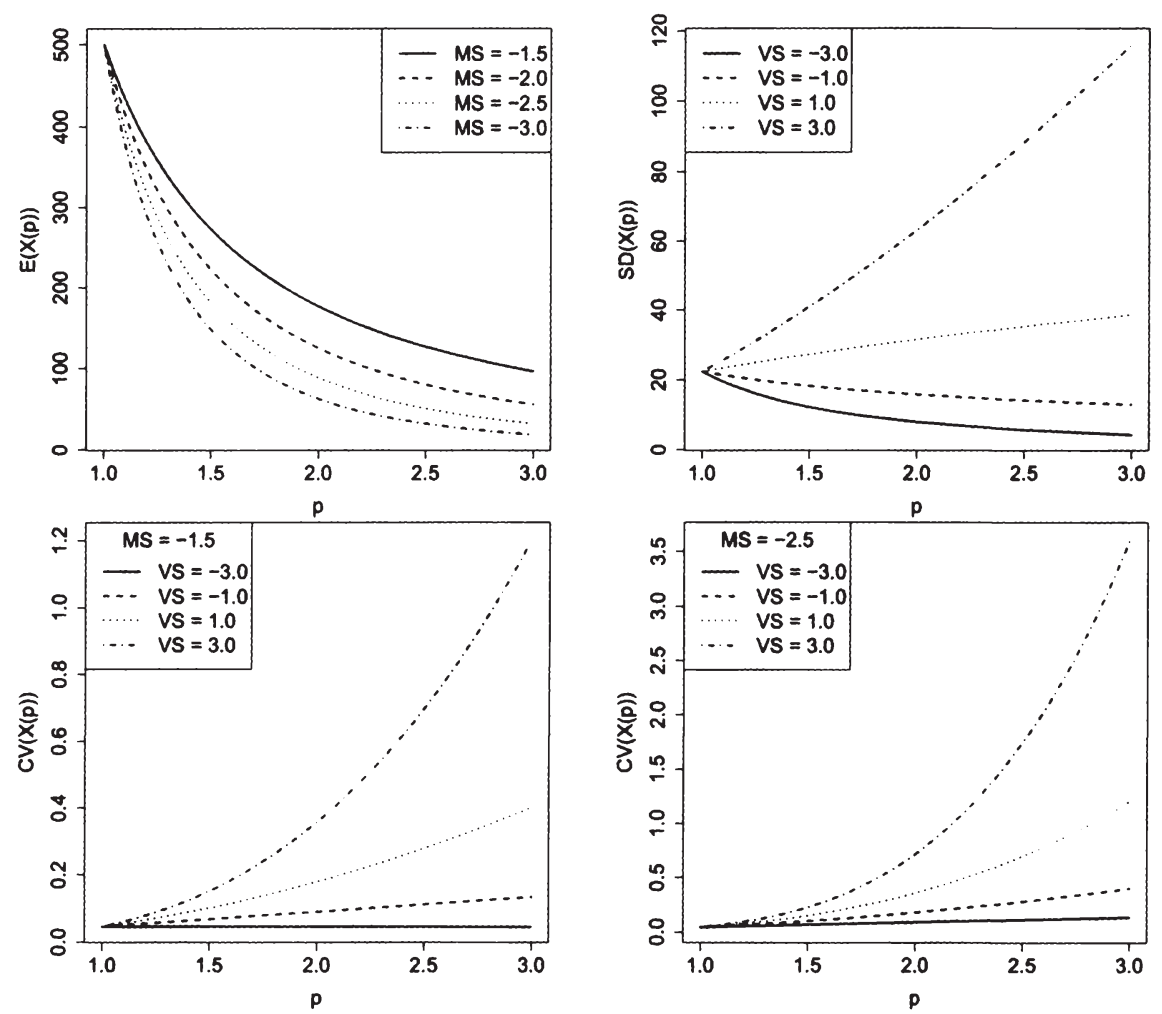

Figure 4.7: Mean, standard deviation, and coefficient of variation under monotone variance

smaller than one. The Gamma distribution with a shape parameter larger than one has increasing failure rate and a shape parameter smaller than one implies a decreasing failure rate (see Barlow and Proschan (1996)).

Optimal policy parameters The lack of failure rate ordering could possibly lead to non-monotone $p^{*}(y)$ (see Theorem 5), but the numerical observations show that $p^{*}(y)$ is still monotone decreasing in $y$. This observation is in line with Remark 1 which states that the failure rate ordering is sufficient but not necessary for monotone $p^{*}(y)$ and it is also sufficient if $p^{*}(y)$ is in the range where $h(p, x)$ is increasing in $p$ for $x \leq y$. The circles on $h(p, y)$ 

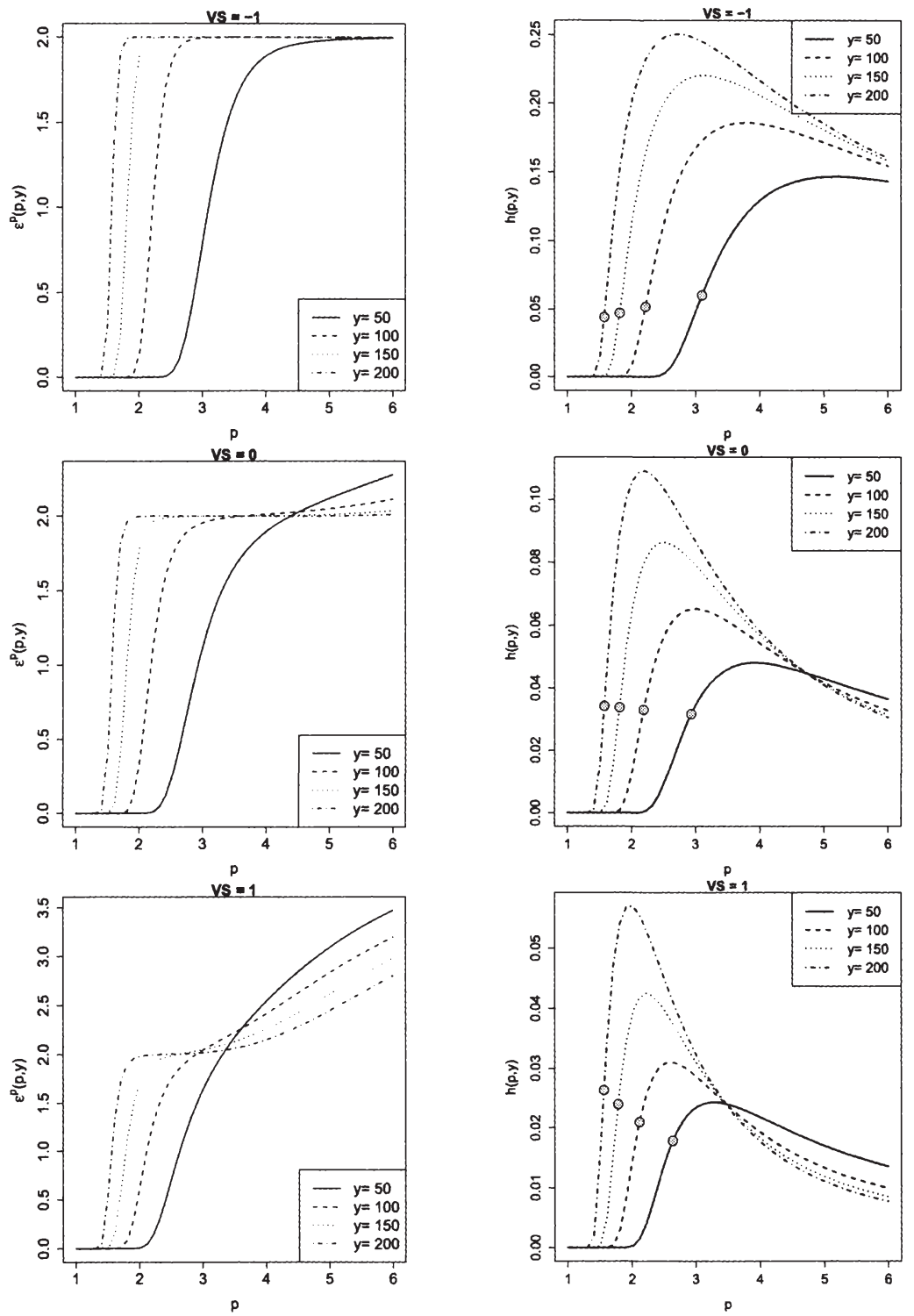

Figure 4.8: $\varepsilon^{p}(p, y)$ and $h(p, y), M S=-2$, monotone variance, circles mark $p^{*}(y)$ 


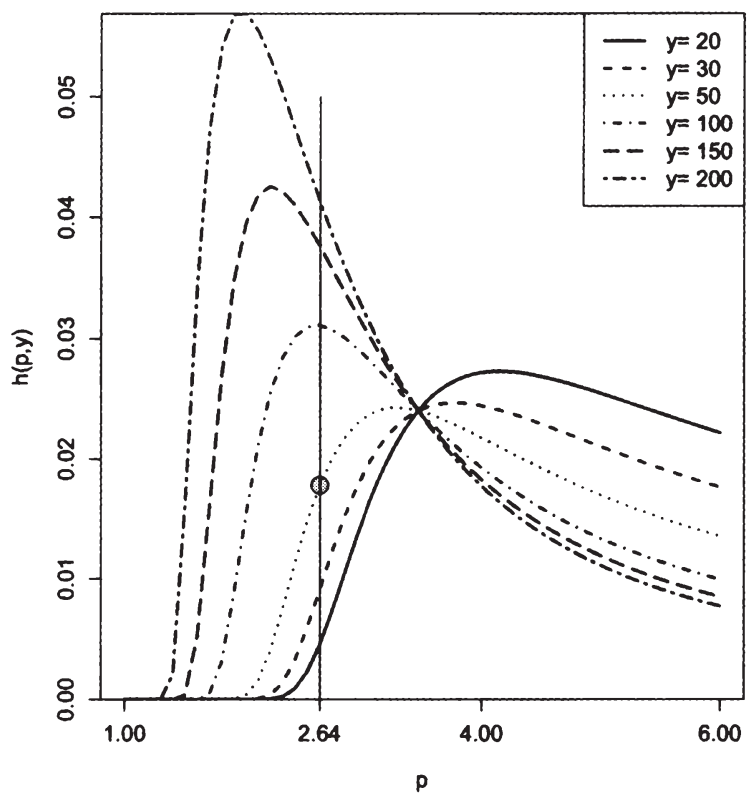

Figure 4.9: $\varepsilon^{p}(p, y)$ for $M S=-2$ and $V S=1$ under monotone variance

curves in Figure 4.8 mark $p^{*}(y)$ for the corresponding $y$ values and they all satisfy the condition mentioned in Remark 1 . Let us describe this condition more in detail in Figure 4.9.

Figure 4.9 shows $\varepsilon^{p}(p, y)$ for $V S=1$ in detail. When we consider a quantity level $y=50$ we see that $p^{*}(50)=2.64$, which is marked. For $x>50, h(2.64, x)$ can be in the increasing as well as decreasing phase with respect to $p$ depending on $x$, e.g. $\left.\frac{\partial}{\partial p} h(p, 200)\right|_{p=2.64}<0$. However, the important part is the curves below $y=50$, i.e. $x \leq 50$. For these curves we see that $h(2.64, x)$ is always in the increasing phase, i.e. $h_{p}(2.64, x) \geq 0$ for all $x \leq 50$. The same structure can be observed for any quantity level $y$, so $h_{p}\left(p^{*}(y), x\right) \geq 0$ for any $x \leq y$, which is enough to satisfy the condition in Remark 1 and to guarantee monotone decreasing $p^{*}(y)$ in $y$.

Figure 4.10 shows the optimal policy parameters for different levels of $M S$ and $V S$. For any $M S$ when $V S=0, \bar{p}$ and $\bar{y}$ are the solutions to the additive model where $\operatorname{Var}(X(p))=500$ independent of $p$. For each $M S$ when $V S=2 M S, \bar{p}$ and $\bar{y}$ are the solutions to the multiplicative model where $C v(X(p))=1 / \sqrt{500}$ independent of $p$. In Figure 4.10 the prices that 

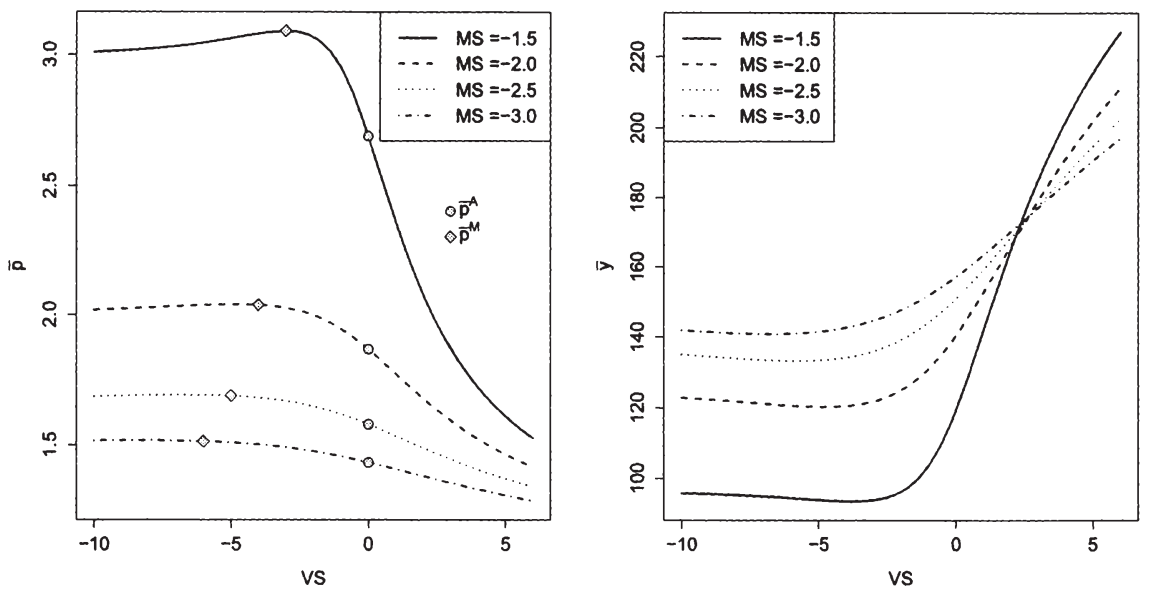

Figure 4.10: $\bar{p}$ and $\bar{y}$ with respect to $V S$ under monotone variance

corresponds to these cases are marked. In line with the theory, the additive model results in lower optimal prices compared to the multiplicative ones.

For a given $M S$, as $V S$ increases $\bar{p}$ decreases since for high $V S$ it is possible to decrease variance by decreasing prices, while for negative $V S$ values, higher prices lead to lower variances. When $M S=-3$, the mean demand is the smallest and it reacts to price changes strongest, and $M S=-1.5$ gives the largest and least sensitive mean demand. As a result, the largest $\bar{p}$ corresponds to $M S=-1.5$ and the smallest to $M S=-3$, which can be seen from the ordering of the $\bar{p}$ curves.

Now the non-monotone order of the $\bar{y}$ curves can be explained in relation to the change of $\bar{p}$ with respect to $V S$. For small $V S, \bar{p}$ of $M S=-1.5$ is much higher than the others, and the difference between each $\bar{p}$ curve is considerably large. As $M S$ increases, there are two competing factors: 1) mean demand increases for a fixed price, 2) $\vec{p}$ increases which might cause the mean demand to decrease. At small $V S$ ranges because of large differences in $\bar{p}$ the second factor is dominating and smaller expected demand leads to smaller order quantity $\bar{y}$. On the other hand, as $V S$ increases, the difference between $\bar{p}$ on each curve decreases. Then, the first factor becomes more important and $\bar{y}$ becomes increasing in $M S$ even if $\bar{p}$ is also increasing. 
Effect of sequential optimization and model misspecification In order to evaluate the effect of sequential optimization we calculate the deterministic optimal price $p^{d}$, which is the price that maximizes $(p-c) E[X(p)]$, and using $p^{d}$ calculate the optimal order quantity $y^{*}\left(p^{d}\right)$. The resulting expected profit is $\Pi\left(p^{d}, y^{*}\left(p^{d}\right)\right)$ and the percentage loss from the maximum expected profit is:

$$
L(D e t)=\frac{\Pi(\bar{p}, \bar{y})-\Pi\left(p^{d}, y^{*}\left(p^{d}\right)\right)}{\Pi(\bar{p}, \bar{y})} 100 .
$$

For each specific $M S$, the optimal price and the order quantity when $V S=0$ refers to the additive solution, $\left(\bar{p}^{A}, \bar{y}^{A}\right)$. Likewise, the optimal policy under the price independent $C v$ is the multiplicative policy with $\left(\bar{p}^{M}, \bar{y}^{M}\right)$. If instead of the optimal policy one of these policies is used there is a loss on the expected maximum profit as:

$$
\begin{aligned}
L(A d d) & =\frac{\Pi(\bar{p}, \bar{y})-\Pi\left(\bar{p}^{A}, \bar{y}^{A}\right)}{\Pi(\bar{p}, \bar{y})} . \\
L(M u l) & =\frac{\Pi(\bar{p}, \bar{y})-\Pi\left(\bar{p}^{M}, \bar{y}^{M}\right)}{\Pi(\bar{p}, \bar{y})} .
\end{aligned}
$$

The loss is the result of using a suboptimal policy which is caused by specifying the demand model incorrectly.

Let us start with the discussion about the effect of sequential optimization. When both $C v$ and $V a r$ are increasing in price, i.e. $V S \geq 0$, for all $M S$ values $p^{d}$ is considerably higher than $\bar{p}$. This is not a surprising result since both of the variability measures can be decreased by decreasing price. When $C v$ is increasing and $V a r$ is decreasing in price, i.e. $V S \leq 0, \bar{p}$ is larger or smaller than $p^{d}$ depending on $M S$. For small values of $M S, \bar{p} \geq p^{d}$ while for large $M S$ we observe the opposite relation $\bar{p} \leq p^{d}$.

Analyzing the effect of sequential optimization shows the relation of several competing effects with price. The two prevalent effects for both the sequential and the integrated approaches are the effects of per unit revenues and demand: Given everything else stays constant per unit revenue is increasing, while demand is decreasing in price which is captured here with the negativity of $M S$. The sequential as well as the integrated optimization balances these two effects. In addition to these two effects, the integrated model considers a third one during optimization, namely the possibility to reduce variability. Adjusting the price (slightly) allows the integrated approach to reduce variance and consequently to increase expected profit. 


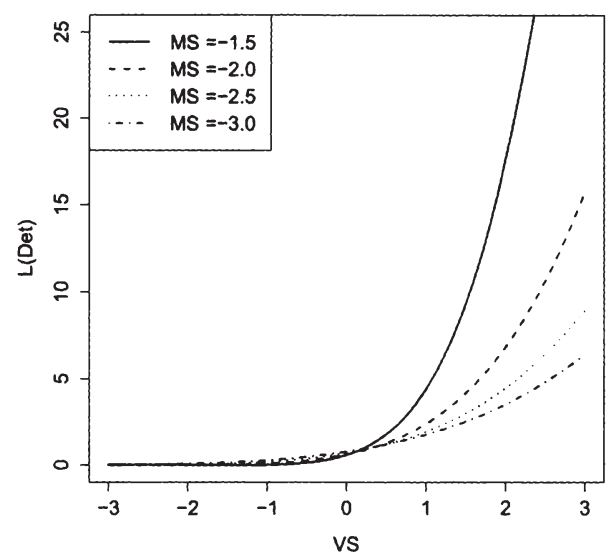

Figure 4.11: Effect of sequential optimization with monotone variance

This effect, however, is in competition with the two effects mentioned before. Hence, the optimization of the integrated model will lead to a balance of all of these three effects.

As it can be seen from Figure 4.11 if variance is an increasing function of price, i.e. $V S>0$, sequential optimization might lead to considerable profit losses which goes almost up to $50 \%$. On the other hand if $V S \leq 0$, the variance is small enough that it can be ignored and the deterministic price performs almost without any loss on profits.

When $V S>0$, the loss is ordered with respect to the mean sensitivity. Considering the price effect on variance during the pricing decision allows using price as a tool to decrease variance. However, when $M S=-3$ the effect of price on the mean demand is quite strong and it does not let flexibility for changing prices in order to decrease the variance, so the mean sensitivity restricts the optimal prices in a smaller range and as a result $L(D e t)$ is not very large. On the other hand, when $M S=-1.5$, there is more room to play with prices in order to manipulate variance and, hence, joint optimization is now able to perform much better than the sequential policy.

Let us now look at the loss resulting from model misspecification. Figure 4.12 shows $L(A d d)$ and $L(M u l)$ for different $V S$ and $M S$. Since the additive model corresponds to $V S=0$, at that point $L(A d d)=0$ for all $M S$. As $V S$ moves away from zero, the loss starts increasing. On the other 

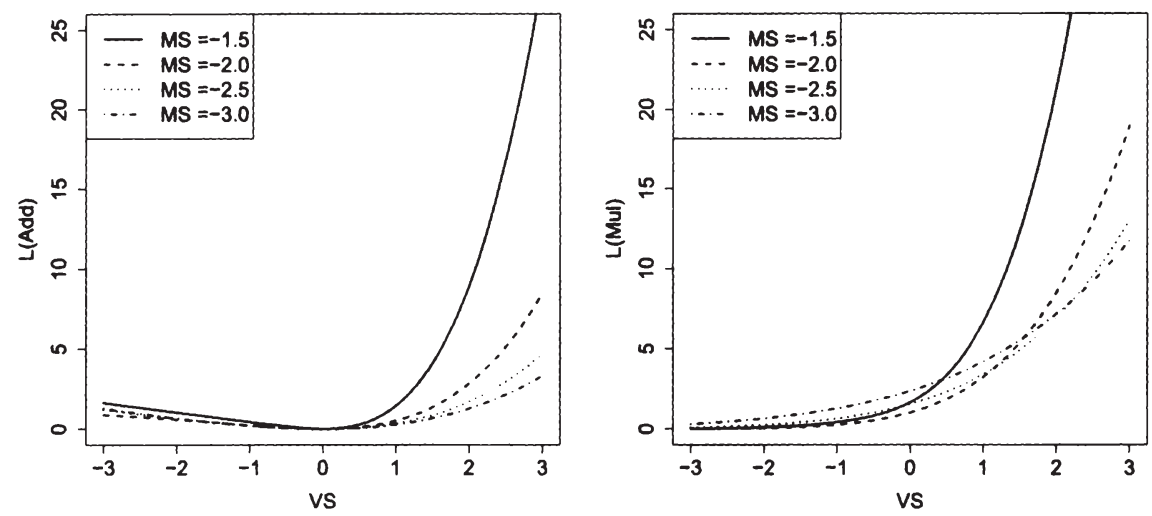

Figure 4.12: Effect of model misspecification with monotone variance

hand, the multiplicative model corresponds to $V S=2 M S$, and the only case that we can see in Figure 4.12 is where $V S=-3$ and $M S=-1.5$. At that point $L(M u l)=0$. For the other values of $M S$ the multiplicative model corresponds to even lower values of $V S$. As for the sequential optimization, both $L(A d d)$ and $L(M u l)$ are ordered with respect to $M S$ as $V S$ gets larger. This result can again be explained by the pricing flexibility.

When $V S>0, L(M u l)$ is generally larger than $L(A d d)$. The multiplicative model assumes a decreasing $V a r$ in price. When the correct model has both increasing $V a r$ and $C v$, the price charged by the multiplicative model becomes too high relative to the correct optimal price. The additive model assumes at least an increasing $C v$ and keeps prices lower in order to avoid high $C v$ s, as a result it resembles more to the correct model. However, the loss can still be quite high especially for large $M S$.

Comparing Figure 4.11 and 4.12 we see that for $V S<0, L(D e t)$ is smaller than $L(A d d)$ and/or $L(M u l)$ for some ranges of $V S$ and $M S$, even though the differences are very small. For $V S>0$, the additive model seems to be the best and the deterministic one performs better than the multiplicative. Hence, we conclude that using a wrong stochastic model might be worse than using the deterministic one. 

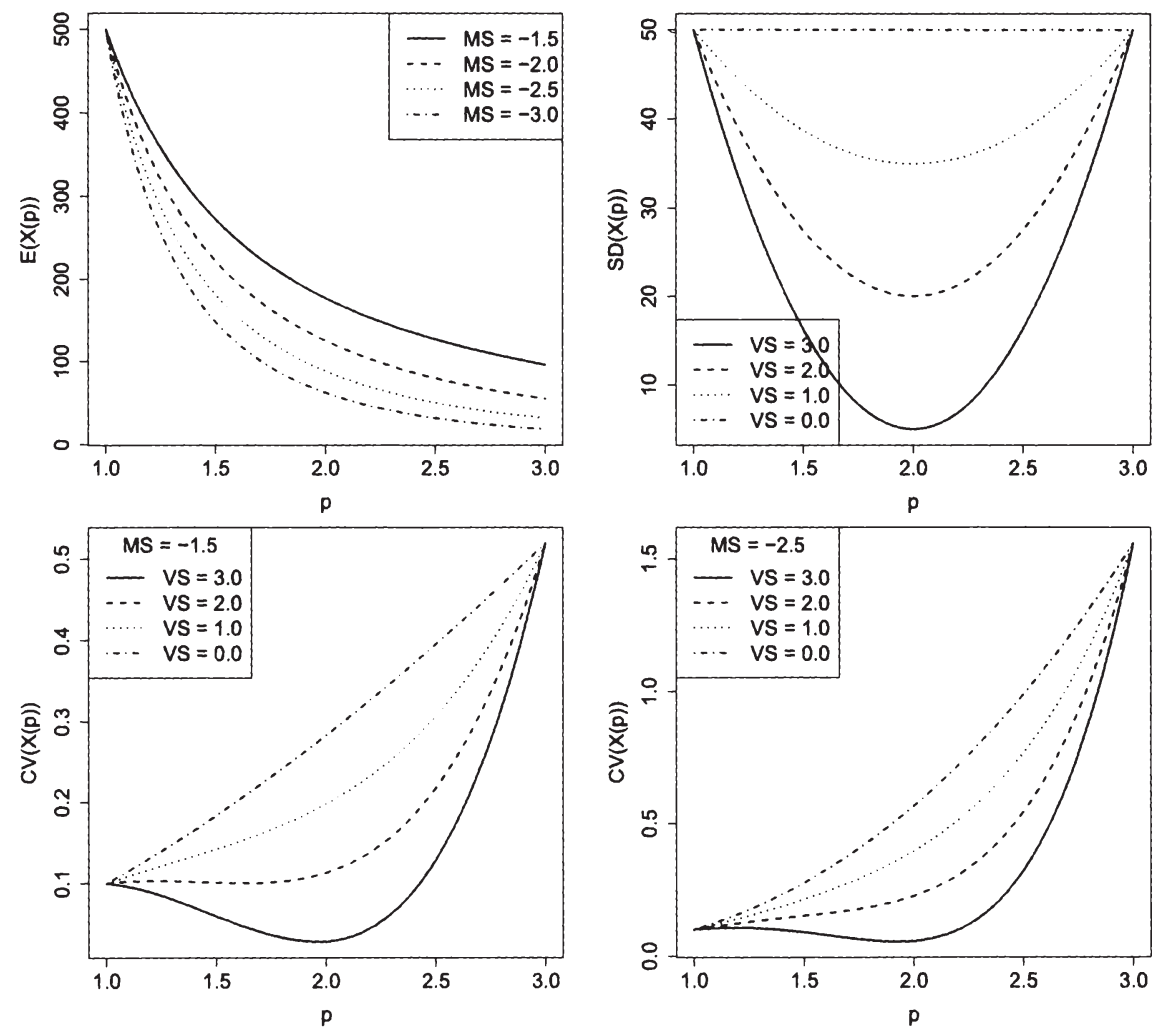

Figure 4.13: Mean, standard deviation, and coefficient of variation under nonmonotone variance

\subsubsection{Non-monotone variance}

In this section we analyze a demand model where the variance is defined as a non-monotone function of price:

$$
\operatorname{Var}(X(p))=\left(15 \operatorname{VS}\left((p-2)^{2}-1\right)+50\right)^{2} .
$$

$V S$ is restricted to be positive, since we want to consider the decreasingincreasing variances. For any $\operatorname{VS}>0, \operatorname{Var}(X(p))$ is increasing in $p$ for small prices and is decreasing for higher ones. For $V S=0, \operatorname{Var}(X(p))$ is again independent of price. The model parameters are presented in Figure 4.13. 

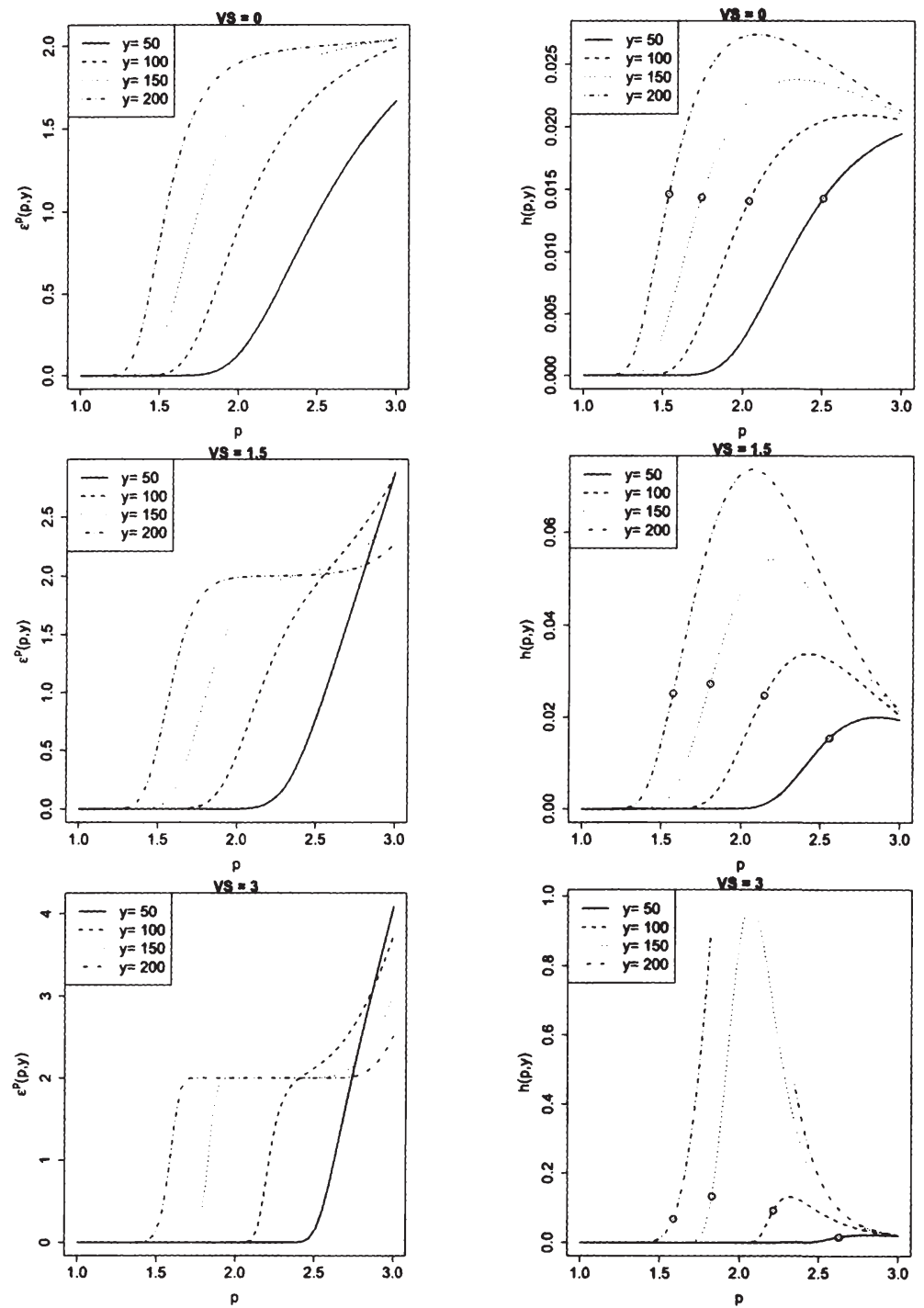

Figure 4.14: $\varepsilon^{p}(p, y)$ and $h(p, y), M S=-2$, non-monotone variance, circles mark $p^{*}(y)$ 

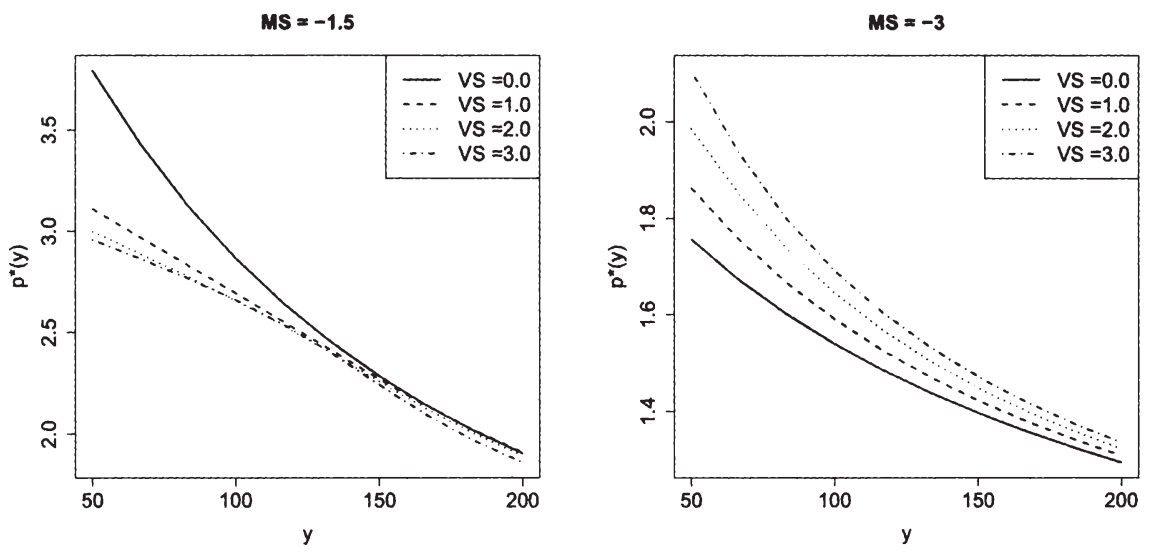

Figure 4.15: $p^{*}(y)$ under non-monotone variance

Sales elasticity and failure rate $\varepsilon^{p}(p, y)$ is again increasing in price (see Figure 4.14) and $h(p, y)$ is not monotone in price, so the failure rate ordering does not exist. On the other hand, unlike the previous example, the IFR property holds for any price level, i.e. $h(p, y)$ increases in $y$, since the shape parameter never becomes smaller than one.

Optimal policy parameters Like the previous example, even if failure rate ordering does not hold, the monotonicity properties are the same as the analytically derived ones under the assumption of failure rate ordering. Specifically the optimal price for a given inventory level $p^{*}(y)$ is decreasing in $y$ and the joint optimal price $\vec{p}$ is increasing in cost $c$ as depicted in Figure 4.15 and Figure 4.16 respectively.

Figure 4.15 shows how the optimal price changes with inventory level. When $M S=-1.5, p^{*}(y)$ decreases in $V S$ but when $M S=-3$ it increases in $V S$ for any $y$, so the curves are ordered in the opposite direction for the two cases. The reason behind this difference is the opposite behavior of the $S D(X(p))$ (so $\operatorname{Var}(X(p))$ ) in the specific ranges of prices where $p^{*}(y)$ belongs to. When $M S=-1.5, p^{*}(y)$ is (almost) always larger than 2 and when $M S=-3$ it is smaller than 2. If we look at Figure 4.13, we can see that at $p=2, S D(X(p))$ reaches its minimum point for all $V S$. On the left of $p=2$ it is decreasing and on the right side it is increasing. When $M S=-1.5, p^{*}(y)$ for any $V S$ is on the right of $p=2$ where smaller prices 

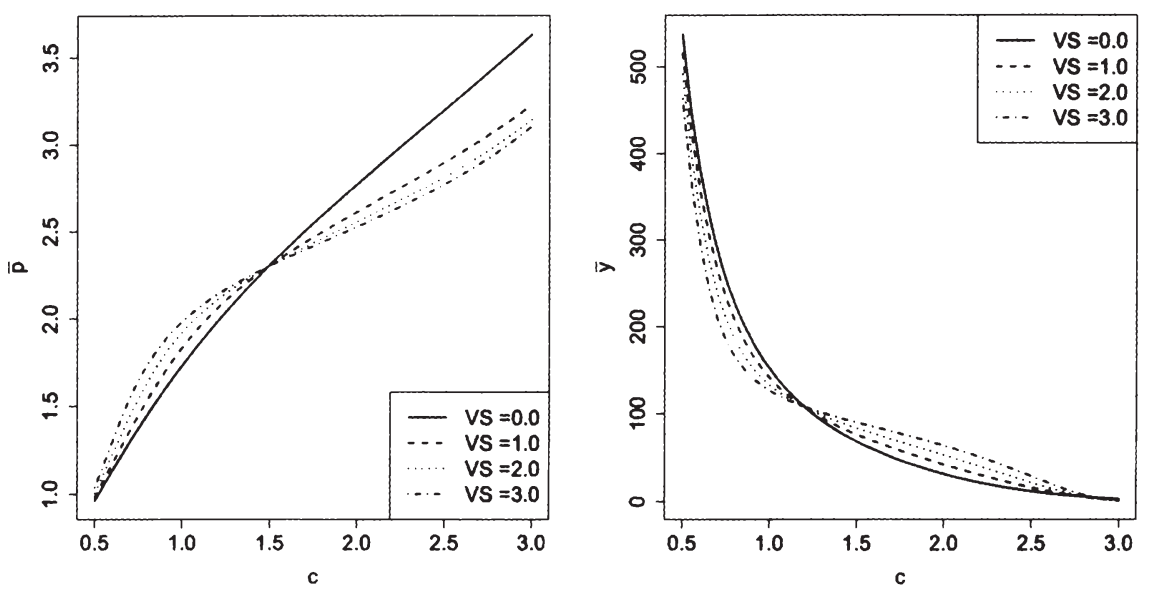

Figure 4.16: $\bar{p}(c)$ and $\bar{y}(c)$ when $M S=-2$ under non-monotone variance

lead to lower variances, and this effect is the strongest for $V S=3$. As a result, $V S=3$ gives the smallest price. On the other hand when $M S=-3$, $p^{*}(y)$ is on the left of $p=2$, where larger prices lead to lower variances. For $V S=3$ the possibility of lowering the variance by increasing price is the largest so $V S=3$ gives the largest price. The complete ordering of $p^{*}(y)$ with respect to $V S$ can be explained using the same argumentation line.

As described in Theorem $10, \bar{p}$ is increasing and $\bar{y}$ is decreasing in $c$ (see Figure 4.16). A closer look at $\bar{p}$ in Figure 4.16 and Figure 4.13 shows that the joint optimal price $\bar{p}$ can be in the decreasing or increasing part of variance depending on $c$. Hence, it is not possible to conclude some property related to $\bar{p}$ and its relation to $S D(X(p))$ or $\operatorname{Var}(X(p))$.

Figure 4.17 shows the optimal price $\bar{p}$ and quantity $\bar{y}$ with respect to $V S$. The optimal prices do not change too much with $V S$, and they change in different directions depending on the $M S$. While $\bar{p}$ is decreasing for $M S=-1.5$ it is increasing for the other $M S$ values. Since for $M S=-1.5$, $\bar{p}$ is generally larger than 2 as $V S$ increases the effect of increasing variance can be compensated by decreasing prices. On the other hand, for smaller $M S$ values, $\bar{p}$ is generally smaller than 2 in which range variance can be decreased by increasing prices.

The order of $\bar{p}$ is similar to the case under monotone variance (Fig. 4.10), as $M S$ increases expected demand for a given price increases, so $\bar{p}$ increases. 

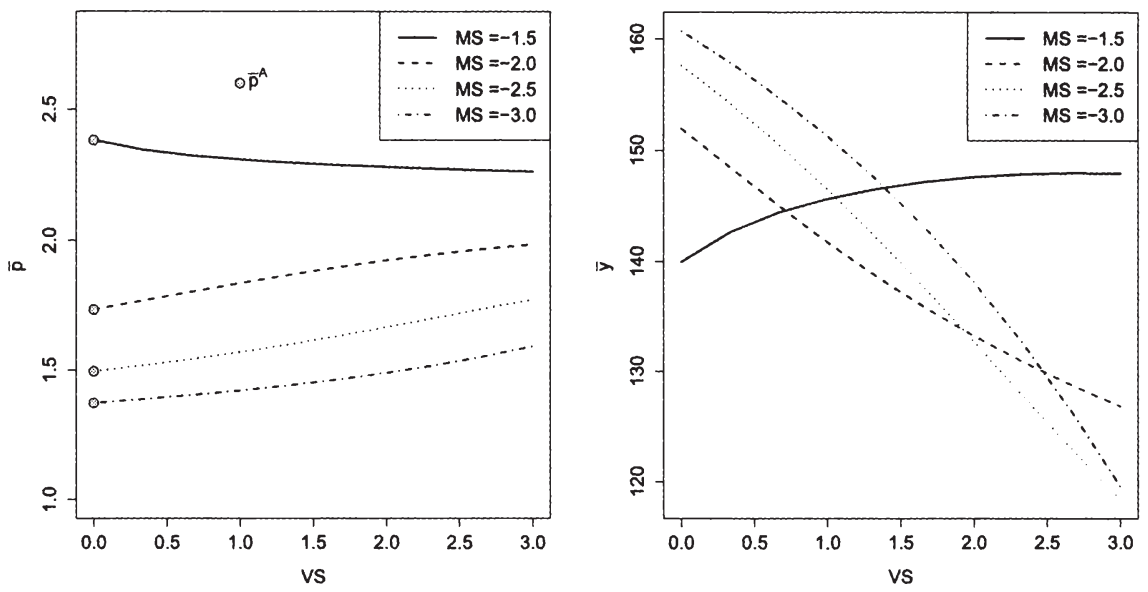

Figure 4.17: $\bar{p}$ and $\bar{y}$ with respect to $V S$ under non-monotone variance

$\bar{y}$ changes in the opposite direction as $\bar{p}$. For $M S=-1.5, \bar{y}$ is decreasing while for the others it is increasing.

Effect of sequential optimization and model misspecification The loss from sequential optimization and model misspecification is shown in Figure 4.18. For the effect of model misspecification we just consider the additive model as the one corresponding to $V S=0$ where the variance is constant in price. None of the $V S$ values can correspond to the multiplicative model which assumes monotone decreasing variance.

The loss from the sequential optimization again depends on the effect of price on mean and variance, and their interaction. As $V S$ increases, 1) variance decreases for each price and as variance decreases the role of considering stochasticity diminishes, 2 ) variance sensitivity increases so the potential of adjusting price to reduce variance increases. If the first effect is stronger the optimal solutions from the sequential and joint optimization come closer, and so $L(D e t)$ decreases. On the other hand, if the second effect is stronger the solutions become more apart and $L(D e t)$ increases. As $V S$ increases the relative power of the two effects are changing which causes the non-monotone shape of $L(D e t)$. Unlike $L(D e t)$ we see that $L(A d d)$ is monotone in $V S$. Since the additive model corresponds to $V S=0$, as $V S$ increases the model becomes more and more different than the additive one. 

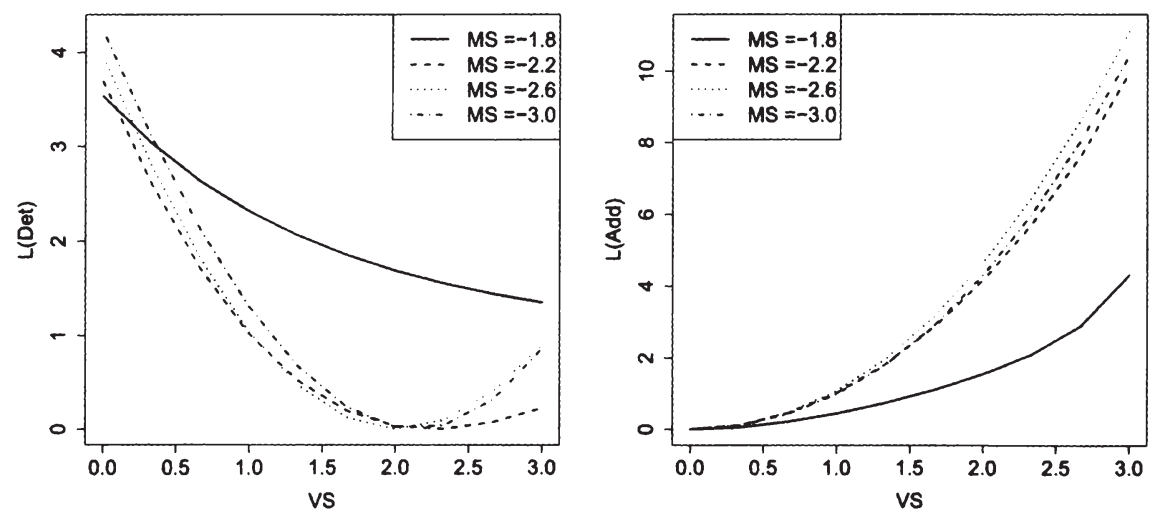

Figure 4.18: Effect of sequential optimization and model misspecification under nonmonotone variance

The most important observation from Figure 4.18 is the difference between $L(D e t)$ and $L(A d d)$. Especially for small $M S$ values $L(D e t)$ is smaller than $L(A d d)$ for a large part of $V S$. If we shift the variance function up without changing its shape, the difference gets even larger. This means that for the pricing decision using a stochastic model which is not correctly defined might be worse than using a deterministic model. 
Emel Arikan - 978-3-631-75394-1

Downloaded from PubFactory at 01/11/2019 05:32:02AM

via free access 


\section{Chapter 5 \\ Conclusion}

In this work we addressed two important managerial decisions, pricing and inventory control, under the price-setting newsvendor model. The majority of the existing literature treats the problem using the two specific demand models, namely the additive and the multiplicative models. The analytical findings differ depending on the demand model employed. Therefore, if we want to gain insights and make use of these findings the crucial point is the correct specification of the demand process, which might not be an easy task.

With an empirical study, we showed that neither the additive nor the multiplicative model is able to represent the demand process considerably better than the other one. In many cases it is not possible to make a choice among the two. Moreover, one has to define some criteria to base the model selection. In order to overcome these difficulties we suggested using a more flexible demand model. The motivation behind the usage of the additive and the multiplicative models within the price-setting newsvendor model is their popularity in the marketing literature and in practice. However, the problems dealt in the marketing literature and the approach do not necessarily overlap with the problem that we are dealing with. In the marketing literature generally several factors influencing demand is treated next to the effect of pricing. Hence, the demand models are generally richer than the ones that we consider in terms of the variables. However, some other aspects of demand, which are crucial in our analysis, are ignored or considered weakly. To be specific, the estimation of the expected demand is not enough for our analysis and we need the complete specification of the demand distribution. With this motivation we suggested modelling the parameters of the demand distribution and as a simple first step we went on with modelling the variance with a more general function which can cover the variance implied by the additive and the multiplicative models. The simulation study that we conducted using the empirical demand values lets us conclude that there is a high potential of improving profits by employing a general model.

Motivated by these facts we formulated the price-setting newsvendor model with a general price dependent demand distribution and continued with the 
analytical study of the optimization problem. The key questions are the conditions leading to a unique profit maximum and the properties of the optimal policy. While for a given price the underlying inventory problem is a concave maximization problem, for the price optimization this holds only for quite restrictive demand formulations. For example, the commonly used iso-elastic demand formulation does not lead to a concave price optimization problem anymore. Since looking for concavity in order to assure a unique optimum is too restrictive we base our analysis on conditions that assure unimodality of the profit function in price.

When the inventory level is fixed, the optimal price is determined by the effect of price on expected revenues. If demand is assumed to be deterministic, the effect of price on demand is reflected on the revenues. Therefore, conditions on price-demand relation, and specifically price elasticity of demand, is the determiner of the optimal price. When we consider the stochasticity and limited inventory, the effect of price on expected sales determines the optimal price. Hence, we introduced the price elasticity of expected sales as a measure which captures this relation. Unimodality is assured as long as the elasticity of expected sales is increasing in price, which we observed for most of the products that we considered in the empirical study. Moreover, if the elasticity of expected sales is increasing in price on the optimal quantity path uniqueness of the joint optimum is also guaranteed. Similarly, we can consider the effect of inventory level on expected sales and introduce the inventory elasticity of expected sales. If we analyze the joint optimization problem on the optimal price path, increasing inventory elasticity of sales guarantees the unique optimum.

The inventory elasticity of sales is strongly related to the properties of the failure rate of demand. If the random demand has increasing generalized failure rate, which is a commonly used assumption in the revenue management and dynamic pricing literature, inventory elasticity is increasing. The structural properties of the optimal price and inventory are also related to the properties of the failure rate. Specifically the monotonicity of the parameters can be assured under failure rate ordering of random demand with respect to price.

We concluded the analysis with a numerical study that illustrates the findings and provides insights to the value of using a general model. The analytical findings continue to hold even when some of the assumptions are relaxed. An important conclusion from the numerical study is that using a deterministic demand model in order to optimize price can be a better strategy than using a stochastic model which is not correctly specified. 
The extensions of this work can be numerous. Each problem setting where the classical price-setting newsvendor model is employed can also be treated with a general demand model. Among those settings the most interesting ones for extensions would be the problems where the demand distribution plays an important role. One such field considers objective functions other than the expected profit. For example mean-deviation criteria or probability of achieving a target profit level are among the objective functions used in the classical newsvendor model. For the former, the specification of the variance is a key issue, and for the latter one the overall shape of the distribution makes a difference. Such formulations are relevant not only as objective function but also as constraints. Hence, for these problems settings, considering a general demand distribution might be valuable.

A relevant extension area for the price-setting newsvendor problem is formulating it as a distribution free problem. In such problems some parameters of the demand distribution is assumed to be known but the complete specification is not required. Therefore, it is not necessary to estimate the complete demand distribution, and estimating the mean and the variance would be enough for the analysis. The objective is not maximizing expected profit but some other criteria such as maximizing the minimum profit or minimizing the ex-post or ex-ante regret. While the price-taking newsvendor problem is studied under the distribution-free approach, combining it with the pricing problem has not been considered yet. For the price-taking newsvendor model, the decision variable, inventory level does not effect the parameters, and the solution procedure roughly considers all possible distributions under these parameters. When pricing decision is included, the parameters are affected by a decision variable, and the analysis would be much challenging with the increased complexity. 
Emel Arikan - 978-3-631-75394-1

Downloaded from PubFactory at 01/11/2019 05:32:02AM

via free access 


\section{References}

Agrawal V and Seshadri S (2000) Impact of uncertainty and risk aversion on price and order quantity in the newsvendor problem. Manufacturing $\mathcal{E}$ Service Operations Management, 2(4):410-423.

Aviv Y and Pazgal A (2007) Optimal pricing of seasonal products in the presence of forward-looking consumers. Working paper, Olin School of Business, Washington University, St. Louis, USA.

Balasubramanian SK and Jain DC (1994) Simple approaches to evaluate competing non-nested models in marketing. International Journal of Research in Marketing, 11:53-72.

Barlow RE and Proschan F (1996) Mathematical Theory of Reliability. SIAM Classics in Applied Mathematics series.

Bernstein F and Federgruen A (2003) Pricing and replenishment strategies in a distribution system with competing retailers. Operations Research, 51(3):409.

Bernstein F and Federgruen A (2005) Decentralized supply chains with competing retailers under demand uncertainty. Management Science, $51(1): 18-29$.

Bitran G and Caldentey R (2003) An overview of pricing models for revenue management. Manufacturing \& Service Operations Management, 5(3):203229.

Bitran G and Mondschein S (1997) Periodic pricing of seasonal products in retailing. Management Science, 43(1):64-79.

Bolton RN (1989) The robustness of retail-level price elasticity estimates. Journal of Retailing, 65(2):193-219.

Boyacı $\mathrm{T}$ and Gallego $\mathrm{G}$ (2002) Coordinating pricing and inventory replenishment policies for one wholesaler and one or more geographically dispersed retailers. International Journal of Production Economics, 77(2):95-111.

Breusch T and Pagan A (1979) A simple test for heteroscedasticity and random coefficient variation. Econometrica, 47:1287-1294. 
Brown JR and Dant RP (2008) Scientific method and retailing research: A retrospective. Journal of retailing, 84(1):1-13.

Cachon G and Swinney R (2009) Purchasing, pricing, and quick response in the presence of strategic consumers. Management Science, 55(3):497-511.

Cachon GP (2003) Supply chain coordination with contracts. In: de Kok A and Graves S (Eds.), Handbooks in Operations Research and Management Science: Supply Chain Management: Design, Coordination and Operation, Elsevier, pp. 229-339.

Chan LMA, Shen ZJM, Simchi-Levi D and Swann JL (2004) Coordination of pricing and inventory decisions: A survey and classification. In: SimchiLevi D, Wu SD and Shen ZJ (Eds.), Handbook of Quantitative Supply Chain Analysis - Modeling in the E-Business Era, chap. 9, Kluwer Academic Publishers, pp. 335-392.

Chen F, Federgruen A and Zheng Y (2001) Coordination mechanisms for a distribution system with one supplier and multiple retailers. Management Science, 47(5):693-708.

Chen X and Simchi-Levi D (2004a) Coordinating inventory control and pricing strategies with random demand and fixed ordering cost: The finite horizon case. Operations Research, 52:887-896.

Chen X and Simchi-Levi D (2004b) Coordinating inventory control and pricing strategies with random demand and fixed ordering cost: The infinite horizon case. Mathematics of Operations Research, 29:698-723.

Chen YF, Ray S and Song Y (2006) Optimal pricing and inventory control policy in periodic-review systems with fixed ordering cost and lost sales. Naval Research Logistics, 53(2):117-136.

Dana JDJ and Petruzzi NC (2001) Note: The newsvendor model with endogenous demand. Management Science, 47(11):1488-1497.

Davidson R and MacKinnon J (2004) Econometric theory and methods. Oxford Univ. Press, New York, NY.

Elmaghraby W and Keskinocak P (2003) Dynamic pricing in the presence of inventory considerations: Research overview, current practices, and future directions. Management Science, 49(10):1287-1309. 
Federgruen A and Heching A (1999) Combined pricing and inventory control under uncertainty. Operations Research, 47(3):454-475.

Fisher M and Raman A (1996) Reducing the cost of demand uncertainty through accurate response to early sales. Operations Research, 44(1):87-99.

Gallego G and van Ryzin G (1994) Optimal dynamic pricing of inventories with stochastic demand over finite horizons. Management Science, 40(8):999-1020.

Granot D and Yin S (2005) On the effectiveness of returns policies in the price-dependent newsvendor model. Naval Research Logistics, 52(8):765779 .

Granot D and Yin S (2007) On sequential commitment in the price-dependent newsvendor model. European Journal of Operational Research, 177(2):939968.

Hanssens DM, Parsons LJ and Schultz RL (2003) Market response models: econometric and time series analysis. Springer.

Hausman WH, Montgomery DB and Roth AV (2002) Why should marketing and manufacturing work together?: Some exploratory empirical results. Journal of Operations Management, 20(3):241-257.

Huh W and Janakiraman G (2008) (s, S) Optimality in Joint InventoryPricing Control: An Alternate Approach. Operations Research, 56(3):783.

Kalyanam K (1996) Pricing decisions under demand uncertainty: A bayesian mixture model approach. Marketing Science, 15(3):207-221.

Karlin S and Carr CR (1962) Prices and optimal inventory policy. In: Arrow KJ, Karlin S and Scarf H (Eds.), Studies in Applied Probability and Management Science, Stanford University Press, CA.

Karmarkar US (1996) Integrative research in marketing and operations management. Journal of Marketing Research, 33(2):125-133.

Karmarkar US and Lele MM (2004) The marketing/manufacturing interface: Strategic issues. In: Chakravarty A and Elishaberg J (Eds.), Managing Business Interfaces: Marketing, Engeneering, and Manufacturing, Springer, pp. 311-328.

Kocabıyıkoğlu A and Popescu I (2005) Joint pricing and revenue management with general stochastic demand. Working paper, INSEAD. 
Koenker R (1981) A note on studentizing a test for heteroscedasticity. Journal of Econometrics, 17(1):107-112.

Koenker R and Bassett G (1982) Robust tests for heteroscedasticity based on regression quantiles. Econometrica, 50:43-61.

Kunreuther H and Richard J (1971) Optimal pricing and inventory decisions for non-seasonal items. Econometrica: Journal of the Econometric Society, $39(1): 173-175$.

Lariviere MA (1999) Supply chain contracting and coordination with stochastic demand. In: Tayur S, Ganeshan R and Magazine M (Eds.), Quantitative Models for Supply Chain Management, Kluwer Academic Publishers, pp. 233-268.

Lariviere MA (2006) A note on probability distributions with increasing generalized failure rates. Operations Research, 54(3):602-604.

Lariviere MA and Porteus EL (2001) Selling to the newsvendor: An analysis of price-only contracts. Manufacturing 85 Service Operations Management, $3(4): 293-305$.

Ledolter $\mathrm{J}$ and Abraham B (2006) Introduction to regression modeling. Duxbury Press, Belmont, CA.

MacKinnon JG, White H and Davidson R (1983) Tests for model specification in the presence of alternative hypotheses. Journal of Econometrics, 21:5370 .

McGill J and van Ryzin G (1999) Revenue management: Research overview and prospects. Transportation Science, 33(2):233-256.

Mills ES (1959) Uncertainty and price theory. Quarterly Quarterly Journal of Economics, 73(1):116-130.

Monahan GE, Petruzzi NC and Zhao W (2004) The dynamic pricing problem from a newsvendor's perspective. Manufacturing \& Service Operations Management, 6(1):73-91.

Montgomery AL and Bradlow ET (1999) Why analyst overconfidence about the functional form of demand models can lead to overpricing. Marketing Science, 18(4):569-583. 
Müller A and Stoyan D (2002) Comparison Methods for Stochastic Models and Risks. Wiley Series in Probability and Statistics, Wiley, West Sussex, England.

Natter M, Reutterer T, Mild A and Taudes A (2007) Practice prize report - an assortment-wide decision-support system for dynamic pricing and promotion planning in DIY retailing. Marketing Science, 26(4):576-583.

Petruzzi NC and Dada M (1999) Pricing and the newsvendor problem: A review with extensions. Operations Research, 47(2):183-194.

Polatoglu H and Sahin I (2000) Optimal procurement policies under price-dependent demand. International Journal of Production Economics, 65(2):141-171.

Polatoglu L (1991) Optimal order quantity and pricing decisions in singleperiod inventory systems. International Journal of Production Economics, 23(1-3):175-185.

Porteus EL (2002) Foundations of Stochastic Inventory Theory. Stanford University Press.

Raz G and Porteus EL (2006) A fractiles perspective to the joint price/quantity newsvendor model. Management Science, 52(11):1764-1777.

Schweitzer ME and Cachon GP (2000) Decision bias in the newsvendor problem with a known demand distribution: Experimental evidence. Management Science, 46(3):404-420.

Shaked M and Shanthikumar J (2007) Stochastic orders. Springer.

Shapiro BP (1977) Can marketing and manufacturing coexist? Harvard Business Review, 55(5):104-114.

Shen $\mathrm{Z}$ and Su X (2007) Customer behavior modeling in revenue management and auctions: A review and new research opportunities. Production and operations management, 16(6):713-728.

Smith SA and Achabal D (1998) Clearance pricing and inventory policies for retail chains. Management Science, 44(3):285-300.

Song Y, Ray S and Boyaci T (2009) Technical note - optimal dynamic joint inventory-pricing control for multiplicative demand with fixed order costs and lost sales. Operations Research, 57(1):245-250, forthcomming. 
Su X and Zhang F (2007) Strategic customer behavior, commitment, and supply chain performance. Working paper, Haas School of Business, University of California, Berkeley, USA.

Talluri KT and van Ryzin GJ (2004) The Theory and Practice of Revenue Management. Kluwer Academic Publishers.

Tellis G (1988) The price elasticity of selective demand: A meta-analysis of economic models of sales. Journal of Marketing Research, 25(4):331-341.

Van Mieghem J and Rudi N (2002) Newsvendor networks: Inventory management and capacity investment with discretionary activities. Manufacturing and service operations management, 4(4):313-335.

Van Mieghem JA and Dada M (1999) Price versus production postponement: capacity and competition. Management Science, 45(12):1631-1649.

Verbeek M (2008) A Guide to Modern Econometrics. John Wiley and Sons, England.

White $\mathrm{H}$ (1980) A heteroskedasticity-consistent covariance matrix estimator and a direct test for heteroskedasticity. Econometrica, 48:817-838.

Whitin TM (1955) Inventory control and price theory. Management Science, 2(1):61-68.

Yano CA and Gilbert SM (2004) Coordinated pricing and production/procurement decisions: A review. In: Chakravarty AK and Eliashberg $\mathrm{J}$ (Eds.), Managing Business Interfaces: Marketing, Engineering and Manufacturing Perspectives, International Series in Quantitative Marketing, chap. 3, Kluwer Academic Publishers, Norwell, Massachusetts, pp. 65-103.

Yao L, Chen YF and Yan H (2006) The newsvendor problem with pricing: Extensions. International Journal of Management Science and Engineering Management, 1(1):3-16.

Young L (1978) Price, inventory and the structure of uncertain demand. New Zealand Operations Research, 6(2):157-177.

Zabel E (1970) Monopoly and uncertainty. Review of Economic Studies, 37(110):205-219.

Zabel E (1972) Multiperiod monopoly under uncertainty. Journal of Economic Theory, 5(3):524-536. 
Ziya S, Ayhan H and Foley R (2004) Relationships among three assumptions in revenue management. Operations Research, 52(5):804-809. 


\title{
Forschungsergebnisse der Wirtschaftsuniversität Wien
}

\author{
Herausgeber: Wirtschaftsuniversität Wien - \\ vertreten durch a.o. Univ. Prof. Dr. Barbara Sporn \\ INFOS UND KONTAKT: \\ WU (Wirtschaftsuniversität Wien) \\ Department of Finance, Accounting and Statistics \\ Institute for Finance, Banking and Insurance \\ Heiligenstädter Straße 46-48, 1190 Wien \\ Tel.: 0043-1-313 36/4556 \\ Fax: 0043-1-313 36/904556 \\ valentine.wendling@wu.ac.at \\ www.wu.ac.at/finance
}

Band 1 Stefan Felder: Frequenzallokation in der Telekommunikation. Ökonomische Analyse der Vergabe von Frequenzen unter besonderer Berücksichtigung der UMTS-Auktionen. 2004.

Band 2 Thomas Haller: Marketing im liberalisierten Strommarkt. Kommunikation und Produktplanung im Privatkundenmarkt. 2005.

Band 3 Alexander Stremitzer: Agency Theory: Methodology, Analysis. A Structured Approach to Writing Contracts. 2005.

Band 4 Günther Sedlacek: Analyse der Studiendauer und des Studienabbruch-Risikos. Unter Verwendung der statistischen Methoden der Ereignisanalyse. 2004.

Band 5 Monika Knassmüller: Unternehmensleitbilder im Vergleich. Sinn- und Bedeutungsrahmen deutschsprachiger Unternehmensleitbilder - Versuch einer empirischen (Re-)Konstruktion. 2005.

Band 6 Matthias Fink: Erfolgsfaktor Selbstverpflichtung bei vertrauensbasierten Kooperationen. Mit einem empirischen Befund. 2005.

Band 7 Michael Gerhard Kraft: Ökonomie zwischen Wissenschaft und Ethik. Eine dogmenhistorische Untersuchung von Léon M.E. Walras bis Milton Friedman. 2005.

Band 8 Ingrid Zechmeister: Mental Health Care Financing in the Process of Change. Challenges and Approaches for Austria. 2005.

Band 9 Sarah Meisenberger: Strukturierte Organisationen und Wissen. 2005.

Band 10 Anne-Katrin Neyer: Multinational teams in the European Commission and the European Parliament. 2005.

Band 11 Birgit Trukeschitz: Im Dienst Sozialer Dienste. Ökonomische Analyse der Beschäftigung in sozialen Dienstleistungseinrichtungen des Nonprofit Sektors. 2006

Band 12 Marcus Kölling: Interkulturelles Wissensmanagement. Deutschland Ost und West. 2006.

Band 13 Ulrich Berger. The Economics of Two-way Interconnection. 2006.

Band 14 Susanne Guth: Interoperability of DRM Systems. Exchanging and Processing XML-based Rights Expressions. 2006.

Band 15 Bemhard Klement: Ökonomische Kriterien und Anreizmechanismen für eine effiziente Förderung von industrieller Forschung und Innovation. Mit einer empirischen Quantifizierung der Hebeleffekte von F\&E-Förderinstrumenten in Österreich. 2006. 
Band 16 Markus Imgrund: Wege aus der Insolvenz. Eine Analyse der Forttührung und Sanierung insolventer Klein- und Mittelbetriebe unter besonderer Berücksichtigung des Konfigurationsansatzes. 2007.

Band 17 Nicolas Knotzer: Product Recommendations in E-Commerce Retailing Applications. 2008.

Band 18 Astrid Dickinger: Perceived Quality of Mobile Services. A Segment-Specific Analysis. 2007.

Band 19 Nadine Wiedermann-Ondrej: Hybride Finanzierungsinstrumente in der nationalen und internationalen Besteuenung der USA. 2008.

Band 20 Helmut Sorger: Entscheidungsorientiertes Risikomanagement in der Industrieuntemehmung. 2008.

Band 21 Martin Rietsch: Messung und Analyse des ökonomischen Wechselkursrisikos aus Unternehmenssicht: Ein stochastischer Simulationsansatz. 2008.

Band 22 Hans Christian Mantler: Makroökonomische Effizienz des Finanzsektors. Herleitung eines theoretischen Modells und Schätzung der Wachstumsimplikationen für die Marktwirtschaften und Transformationsőkonomien Europas. 2008.

Band 23 Youri Tacoun: La théorie de la valeur de Christian von Ehrenfels. 2008.

Band 24 Monika Koller: Longitudinale Betrachtung der Kognitiven Dissonanz. Eine Tagebuchstudie zur Reiseentscheidung. 2008.

Band 25 Marcus Scheiblecker: The Austrian Business Cycle in the European Context. 2008.

Band 26 Aida Numic: Multinational Teams in European and American Companies. 2008.

Band 27 Ulrike Bauernfeind: User Satisfaction with Personalised Internet Applications. 2008.

Band 28 Reinhold Schodl: Systematische Analyse und Bewertung komplexer Supply Chain Prozesse bei dynamischer Festlegung des Auftragsentkopplungspunkts. 2008.

Band 29 Bianca Gusenbauer: Öffentlich-private Finanzienung von Infrastruktur in Entwicklungslăndern und deren Beitrag zur Armutsreduktion. Fallstudien in Vietnam und auf den Philippinen. 2009.

Band 30 Elisabeth Salomon: Hybrides Management in sino-österreichischen Joint Ventures in China aus österreichischer Perspektive. 2009.

Band 31 Katharina Mader. Gender Budgeting: Ein emanzipatorisches, finanzpolitisches und demokratiepolitisches Instrument. 2009.

Band 32 Michael Weber. Die Generienung von Empfehlungen für zwischenbetriebliche Transaktionen als gesamtwirtschaftliche Infrastrukturleistung. 2010.

Band 33 Lisa Gimpl-Heersink: Joint Pricing and Inventory Control under Reference Price Effects. 2009.

Band 34 Erscheint nicht.

Band 35 Dagmar Kiefer: Multicultural Work in Five United Nations Organisations. An Austrian Perspective. 2009.

Band 36 Gottried Gruber: Multichannel Management. A Normative Model Towards Optimality. 2009.

Band 37 Rainer Quante: Management of Stochastic Demand in Make-to-Stock Manufacturing. 2009.

Band 38 Franz F. Eiffe: Auf den Spuren von Amartya Sen. Zur theoriegeschichtlichen Genese des Capability-Ansatzes und seinem Beitrag zur Armutsanalyse in der EU. 2010. 
Band 39 Astrid Haider: Die Lohnhöhe und Lohnstreuung im Nonprofit-Sektor. Eine quantitative Analyse anhand ősterreichischer Arbeitnehmer-Arbeitgeber-Daten. 2010.

Band 40 Maureen Lenhart: Pflegekräftemigration nach Österreich. Eine empirische Analyse. 2010.

Band 41 Oliver Schwank: Linkages in South African Economic Development. Industrialisation without Diversification? 2010.

Band 42 Judith Kast-Aigner: A Corpus-Based Analysis of the Terminology of the European Union's Development Cooperation Policy, with the African, Caribbean and Pacific Group of States. 2010.

Band 43 Emel Arikan: Single Period Inventory Control and Pricing. An Empirical and Analytical Study of a Generalized Model. 2011.

www.peterlang.de 
Emel Arikan - 978-3-631-75394-1

Downloaded from PubFactory at 01/11/2019 05:32:02AM

via free access 
Emel Arikan - 978-3-631-75394-1

Downloaded from PubFactory at 01/11/2019 05:32:02AM

via free access 